\title{
INVESTIGATION OF THE MECHANISM IN RIJKE PULSE COMBUSTORS WITH TANGENTIAL AIR AND FUEL INJECTION
}

Final Report

By B. T. Zinn, J. I. Jagoda, B. R. Daniel, and T. Bai

March, 1993 DOE/AL/31881 T 22

\author{
Work Performed Under Contract No. DE-ASO4-85AL31881
}

For

U.S. Department of Energy

Albuquerque Operations Office P.O. Box 5400

Albuquerque, New Mexico 87115

By

School of Aerospace Engineering Georgia Tech Research Corporation

Georgia Institute of Technology

Atlanta, Georgia 30332

\section{DISCLAIMER}

This report was prepared as an account of work sponsored by an agency of the United States Government. Neither the United States Government nor any agency thereof, nor any of their employees, makes any warranty, express or implied, or assumes any legal liability or responsibility for the accuracy, completeness, or usefulness of any information, apparatus, product, or process disclosed, or represents that its use would not infringe privately owned rights. Reference herein to any specific commercial product, process, or service by trade name, trademark, manufacturer, or otherwise does not necessarily constitute or imply its endorsement, recommendation, or favoring by the United States Government or any agency thereof. The views and opinions of authors expressed herein do not necessarily state or reflect those of the United States Government or any agency thereof.

TECHNICAL INFORMATION CENTER

OFFICE OF SCIENTIFIC AND TECHNICAL INFORMATION

UNITED STATES DEPARTMENT OF ENERGY 


\section{DISCLAMIER}

Portions of this document may be illegible in electronic image products. Irrages are produced from the best available original docoment. 


\section{Project Summary}

The three year's research, under DOE Contract No. DE-AS04-85AL31881, focuses on the investigation of the mechanisms that control the driving of pulsations in the liquid fuel burning, Rijke type, pulse combustor developed under a preceding DOE contract.

The combustor consists of a vertical steel tube with two decouplers at both ends. Most of the combustion air and all the fuel enter the combustor through a developed air/fuel injection system, which is attached to the combustor tangentially and generates a swirling flow inside the combustor tube.

To study the mechanisms that control the operation of this combustor, an experimental setup is developed with access for detailed optical measurements. Propane is employed as fuel because the absence of liquid drops and combustion generated particulates in the combustion region significantly simplifies the optical diagnostics. The experimental techniques utilized include acoustic pressure measurements, space and time resolved radiation measurements, steady temperature measurements, exhaust flow chemical analysis, high speed video and intensified images of the reacting flow field by a computer based CCD camera imaging system.

Flow visualization by the imaging system and the results from radiation intensity distribution measurements suggest that the periodic combustion processes caused by periodic vortex shedding and impingement provide the energy required to sustain the pressure oscillations. High radiation intensity occurs during a relatively short period of time and is in phase with the pressure oscillations, indicating that Rayleigh's criterion is satisfied.

Periodic variations of the air and fuel flow rates and, consequently, the air/fuel ratio of the reacting mixture inside the combustor appear to be another mechanism that contributes to the occurrence of periodic combustion and heat release processes. The presence of this mechanism has been uncovered by acoustic pressure measurements that revealed the presence of travelling pressure waves inside the air and fuel feed lines. These travelling waves produce periodic fuel and air feed rates which, in turn, result in periodic combustion and heat release processes within the combustor. 


\section{TABLE OF CONTENTS}

Page

INTRODUCTION 2

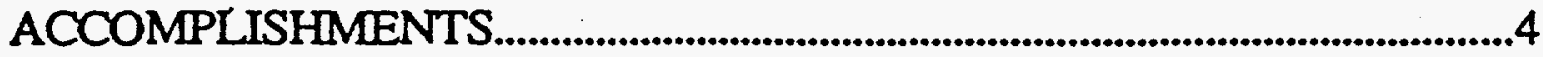

Task I

Development of the Rijke Type Pulse Combustor......................4

Task II

$\therefore \quad$ Development of the Intensified Imaging System......................7

Task III

Radiation Measurement

Task IV

Visualization Studies ..........................................................................11

Driving Mechanism Studies .........................................................13

Laser Doppler Velocity Measurements....................................18

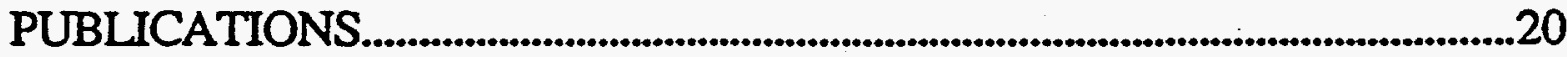

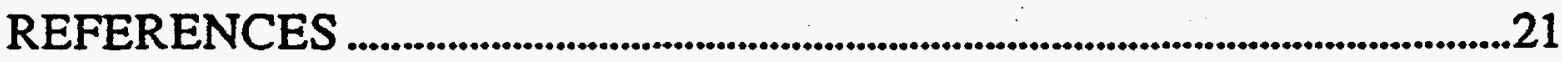




\section{INTRODUCTION}

This report summarizes the accomplishments of DOE Contract No. DEASO4-85AL31881. This three year investigation started in August 1989 and its objective was to elucidate the mechanisms that control the driving of pulsations in the liquid fuel burning, Rijke type, pulse combustor developed under a preceding DOE contract ${ }^{1}$, see Fig. 1. It was demonstrated in that contract that the developed Rijke type pulse combustor can burn a variety of light and heavy liquid fuel oils with high combustion efficiencies while using low excess air, which produces high thermal efficiencies.

Since the elucidation of the driving mechanism in the Rijke pulse combustor required the use of optical diagnostics (e.g., radiation measurements), it was decided to perform these investigations in a Rijke pulse combustor that burned propane instead of a liquid fuel in order to avoid difficulties that are often encountered due to the presence of liquid droplets in the combustion region. Consequently, an effort was made to develop a Rijke pulse combustor that is similar to the one developed in the preceding program and demonstrated similar performance characteristics. Such a pulse combustor was developed in the early phases of this program, and it is shown in Fig. 2. The developed experimental setup was provided with capabilities for measuring steady combustor temperature distributions, the characteristics of the excited pressure oscillations, the exhaust flow composition, the characteristics of the flow field and the reaction rates. This pulse combustor consists of a cylindrical tube that is attached to a decoupling chamber at each end. Fuel and air are supplied via a tangential air/fuel injection system that is located at a distance of $\mathrm{L} / 4$ from the combustor entrance, where $\mathrm{L}$ is the combustor length. Part of the combustor tube, where combustion occurs, is water cooled. This section is also equipped with flat quartz windows to permit optical diagnostics. Two windows are installed on the walls of the rectangular injection duct that supplies the reactants into the combustor tube and additional windows are installed on the walls of the combustor tube section, in the immediate vicinity of the reactants injection system.

This setup was first used to investigate the characteristics of the combustion region and the unsteady heat release process. The former was investigated with the aid of an intensified imaging system that measured the spatial distribution of emitted radiation. It revealed the presence of radiation patterns having the 
appearance of large coherent vortex structures immediately downstream of the air/fuel injection plane. The visualization studies were complemented by measurements of the time and space dependencies of radiation intensities, which were used to determine the characteristics of the investigated combustion process and heat release rates. These showed that the reaction and heat release processes were periodic and in phase with the pressure oscillation, which satisfied Rayleigh's criterion for driving the pressure oscillations by a combustion process.

The setup was then used to investigate the mechanisms responsible for the driving of the pulsations. This study revealed the presence of traveling acoustic waves in both the fuel and air feed lines during pulse combustor operation. These traveling waves are induced by the pressure oscillation in the combustor and they propagate in a direction opposite to that of the air and fuel flows. Calculations based upon measured data showed that these traveling waves produce oscillatory fuel and air injection rates that, in turn, produce periodic variations of the air/fuel ratio and reaction rate inside the combustor. Pulse combustion operation occurred when the oscillatory heat release process had a component in phase with the pressure oscillation.

To verify that the modulation of the fuel and air supply rates by the combustor pressure oscillations was an important element of the driving mechanism, the combustor performance was investigated under operating conditions that prevented wave propagation in the fuel and air supply systems. This was accomplished by choking the flow leaving either the fuel or the air supply line, or both. These tests provided improved understanding of the driving mechanism.

Finally, an investigation of the velocity field in the combustion zone was initiated, and is currently in progress. This study uses a Laser-Doppler Velocimeter (LDV) system to determine the characteristics of the steady, acoustic and turbulent velocity components in specific regions of the combustion zone. The objective of these measurements is to obtain velocity data that could be used to improve the understanding of pulsating combustion processes, and verify a previously advanced hypothesis regarding the characteristics of the flow in the combustion region, which is needed to complete the description of the driving mechanism.

This research program has been divided into the following five tasks: (I) development of a Rijke pulse combustor setup suitable for detailed investigation of combustor performance and driving mechanism, (II) determination of the 
combustion zone structure and reaction rates characteristics by use of an intensified imaging system, (III) determination of the flow characteristics of the combustion zone by use of various visualization techniques, (IV) determination of reaction rates by radiation measurement, and (V) determination of the combustion zone velocities by use of LDV. The accomplishments of the four tasks are briefly discussed in the remainder of this report while the results of the fifth task will be discussed in a following report after this task is completed.

\section{ACCOMPLISHMENTS}

\section{Task I: Development of the Rijke Pulse Combustor}

\section{Determination of Combustor Design Parameters and Configuration}

Initial efforts under this contract were concerned with the development of a pulse combustor setup that was suitable for the proposed investigations of the performance driving mechanisms of the previously developed liquid fuel burning Rijke pulse combustor ${ }^{1}$, see Fig. 1 . These objectives required that the developed pulse combustor be geometrically similar to the previously developed Rijke pulse combustor, that it possess optical access for visualization studies, and capabilities for measuring radical radiation, velocities, temperatures and pressures. Finally, the developed Rijke pulse combustor was designed to burn gaseous fuels in order to eliminate interference from liquid drops and combustion generated particulates in the combustion region with the proposed optical diagnostics.

First, to determine whether the previously developed pulse combustor could burn propane and whether its performance while burning propane was similar to its performance while burning liquid fuels, the liquid fuel burning Rijke pulse combustor was modified to operate on propane. Various gas/air injection systems were tested, and the results were used to guide the design and development of the new pulse combustor setup. In addition, the systematic study of different injector configurations resulted in a better understanding of the driving mechanisms in this pulse combustor.

This study also investigated the performance of the pulse combustor when it was retrofitted with various injection systems that burned the reactants in premixed or diffusion type flames, see Figs 3 and 4 . In addition, tests were carried out with the reactants injected tangentially or radially into the combustor tube. 
In the premixed fuel injection system, see Fig. 3, two fuel jets are injected normally into an air stream through openings in the side walls of a small mixing cavity. The resulting mixture enters a rectangular duct of width, height and length of $1.6 ", 3.6 "$ and $6.0 "$ ", respectively. Combustion generally started in the mixing duct and it was completed in the combustor tube. Tests were conducted with the reactants entering the combustor tube either tangentially or radially, with different air/fuel ratios. The results are discussed in Ref. 2.

In the diffusion flame injection system, see Fig. 4, the mixing cavity was removed and the fuel and air were injected into the rectangular duct through two concentric tubes with the fuel tube located inside the larger air tube. This injection system was tested with the reactants entering the combustor tube either tangentially or radially. The results are presented in Ref. 2.

Additional tests investigated the dependence of the combustor performance upon the combustor length (which was varied between 60 and 120 inches), the boundary conditions (e.g., the effect of closing the upper end of the combustor, which eliminated the axial air flow and established a "solid wall" acoustic boundary condition at the upper end of the combustor, see Fig. 5), the location of air and the fuel injection system (e.g., see Fig. 6), and the manner in which the air/fuel mixture enters the combustor (e.g., see Fig. 7).

\section{Development of the Pulse Combustor Setup}

A schematic and a photograph of the pulse. combustor and its two supports are presented in Figs. 8 and 9, respectively. The developed pulse combustor is operated in a horizontal rather than the vertical orientation in which the original pulse combustor, see Fig. 1, was operated. This facilitates mounting and traversing of the pulse combustor in the optical diagnostic station without affecting its performance characteristics. The pulse combustor consists of several steel pipe sections, a water cooled reactants injection and a combustion section that is equipped with quartz windows to permit optical diagnostics, and two decoupling chambers. Each decoupler is resting on a support and translation system that can move the combustor along three, mutually perpendicular, axes, see Fig. 10. Using this support arrangement, any combustor section can be moved onto the probe volume of the measurement system. The operation of the developed pulse combustor setup is controlled by the reactants supply systems, ignition system, safety system and the control panel shown in Fig. 11. 
Since most of the planned measurements required optical access into the injection and combustion section, this section was fitted with strategically placed, flat windows, see Figs. 8 and 12. The rectangular injection duct that supplies the reactants into the combustor tube has optical windows on two of its walls, and it is attached to the combustor tube in a manner that forces the reactants to be injected tangentially into the combustor. Two additional round windows whose diameter equals that of the combustor tube are installed at the end walls of the two decouplers to allow viewing the combustion process along the axis of the combustor tube. The combustor is also equipped with 21 measurement ports along its axis, which are used for steady temperature and dynamic pressure measurements. Finally, the developed combustor permits the employment of different fuel injection systems and different fuels.

This combustor can be operated with energy input rates up to 400,000 $\mathrm{Btu} / \mathrm{Hr}$. Since most of this energy is released in the vicinity of the injection point, this section of the combustor is cooled by six water jackets, see Fig. 13. Those water jackets are connected into two groups of pipes with approximately equal flow resistance to keep the same cooling water flow rates through the two groups of water jackets.

The combustor is ignited with an oxygen/propane pilot flame generated by an ignitor specifically designed for this system. The ignitor is mounted on the side wall of the rectangular duct and it is constructed of a stainless steel cylindrical combustion chamber with a spark plug threaded into its end wall, see Fig. 12. Ignition is attained by turning the spark plug on and feeding small flow rates of oxygen and propane into the cylindrical chamber. These streams mix and the resulting mixture is ignited by the spark plug. The resulting flame enters the rectangular injection duct through a $1 / 4$ " diameter hole. Once this flame is established, a stream of reactants is injected into the rectangular duct where it is ignited by the oxygen/propane flame.

To avoid an explosion, a rupture disc has been installed on the upstream decoupler. This rupture disc is designed to break and vent the system once the pressure inside exceeds a certain threshold. The safety system also employs a detector which monitors the UV radiation from the combustion zone and is programmed to cutoff the fuel flow to the combustor as soon as combustion there stops. This prevents the formation of an explosive mixture inside the combustor after an accidental flame extinguishment. 
Generally, the mode of operation of the combustor were determined from measured acoustic pressures, which were measured with Kistler model 211B5 piezoelectric pressure transducers. Each transducer has been mounted on a "semi-infinite" tube to prevent heat damage to the pressure transducer from the hot gases and flames inside the combustor, and to provide a measuring probe with a flat frequency response. In this arrangement, the pressure transducer is mounted at the end of a 2 feet long .25" diameter stainless steel tube which is attached at one end to the combustor wall and to a plastic tube which simulates a "semi-infinite" tube at its other end. The pressure transducers are calibrated before a test together with their "semi-infinite" probes. A high-level, low impedance output signal with the resolution on the order of one part per 20,000 of full scale range is generated by the utilized pressure transducers with a sensitivity independent of cable length or capacitance. The measured signals are amplified by a Kistler model 504E Duel Mode Amplifier, and the variable gain output of this amplifier ensures voltage levels adequate for computer based data acquisition.

\section{Task II: Development of the Intensified Imaging System}

\section{System Description}

The intensified imaging system was used to obtain intensified, gated, images of flame radiation measurements and for flow visualization. The imaging system basically consists of a intensified CCD camera and a computer system. The CCD camera was supported by a tripod at a distance of approximately two feet from the quartz window. A Nikon $28 \mathrm{~mm}$ wide angle lens was used to focus the observed object onto the image plane, the CCD array plane. The spatial resolution of the camera is $128 \times 128$ pixels with an adjustable temporal resolution down to 50 nanoseconds. In addition, up to four channels of analog data can be acquired simultaneously with the images.

A technique has been developed in which the pressure signal recorded simultaneously with the images is used as a clock. This permits recording of images at the same phase in the cycle during successive cycles in order to determine the cycle to cycle repeatability of the combustion process. In addition, images obtained at different instances over many cycle can be ensemble averaged and sorted into chronologically sequential events describing the location of reignition and the shape of the flame spread in order to quantify the local flame 
speed at different instances during the cycle by calculating the phase angle between the local radiation and pressure fluctuations.

\section{Measurements}

The intensified imaging system has been used to obtain $\mathrm{C}-\mathrm{H}$ radical radiation and broad band visible radiation data, which are proportional to the reaction rates at the measurement locations. The utilized setup is shown in Figs. 14 and 15. During the tests, an acoustic pressure signal measured in the duct was amplified and sent into the computer through one of four analog signal input ports. This pressure signal was used as a clock to determine the phase of the image relative to the pressure oscillation. In most of the tests, the sampling rate of the imaging system was set at 503 frames per second and for each frame the exposure time was 50 microsecond. These tests were used to obtain distributions of the $\mathrm{C}-\mathrm{H}$ and the broad band visible radiation intensity when the combustor oscillated at its fundamental mode and its first harmonic, and when the combustor was operated in a steady mode. In the following, some of the results are briefly discussed. More details can be found in Ref. 2 and 3.

Sequences of the broad band visible radiation intensity distribution images obtained in different cycles are presented in Figs. 16 through 20. These images were obtained when the $99 \mathrm{~Hz}$ fundamental mode oscillation was excited in the combustor. The combustor was operated with a fuel input rate of 0.94 SCFM and nondimensional air/fuel ratio $\alpha$ of 1.35 . The system sampling rate was approximately 5 frames per cycle. These images show the instantaneous distributions of the radiation intensity and their changes during a cycle. An examination of the images in Figs. 16-20 suggests that during a given cycle combustion begins inside two small, vortex-like, structures that grow in size as they are convected downstream. These structures merge with each other at a distance (approximately) $Y=7 D$, where $D$ is the injection duct diameter, downstream of the entrance to the injection duct, resulting in intensification of the rate of combustion to its maximum level. The reaction rate decreases during the remainder of the cycle to the point where only very low radiation is present at the end of the cycle. This process repeats itself from one cycle to another, see Figs. 16 through 20.

More detailed information about the evolution of the vortex-like structures and the periodic combustion process in the air/fuel injection duct was obtained by using the imaging system in a stroboscopic mode. This technique was developed 
earlier under this program 2 . The images obtained using this technique under the same operating conditions as those discussed above are presented in Fig. 21. It provides 25 views for one cycle. An analysis of these images supports the conclusions derived from the above discussed images.

Broad band visible radiation measurements were also conducted when the combustor was operated in a steady mode (i.e., no pulsations were present in the system). The images obtained under this operating conditions, using a 503 frames per second sampling rate, are presented in Fig. 22. An examination of these images indicates that no coherent flow structures are present in the combustion zone and that the combustion process has the characteristics similar to those of a turbulent, steady, jet flame. Furthermore, similar radiation intensity distributions appear in all the images, suggesting the lack of any periodic phenomenon.

\section{Task III: Radiation Measurements}

\section{Development of the Radiation Measurement System}

An optical system for measuring the total radiation emitted by the combustion zone (i.e., global), and that emitted from specific regions was developed, see Figure 23. These data were then used to determine the reaction rates under various operating conditions. A photomultiplier tube, Hamamatsu model R-268, is used to measure the radiation emitted from the flame because of its high frequency response and broad band spectral response. The photomultiplier views the combustor section that has quartz windows through an optical arrangement that permit measurement of the radiation passing through a local area of $0.7 " \times 0.7 "$. The radiation measurement system is mounted on a translation mechanism that can move in two direction and permits measurements of the $\mathrm{X}$ - and $\mathrm{Y}$-dependencies of the flame radiation. By choice of appropriate filters, $\mathrm{C}-\mathrm{C}, \mathrm{C}-\mathrm{H}$ and $\mathrm{O}-\mathrm{H}$ radiation can be measured.

\section{Measurements}

In this section typical measured $\mathrm{C}-\mathrm{H}$ radiation data is discussed; detailed discussions of all the measurements can be found in Refs. 1 and 2. The data discussed in this section was obtained in tests with the premixed air/fuel injection system, a propane input rate of 1.6 SCFM and nondimensional air/fuel ratio $\alpha$ of 0.97 . The results are presented in Figures 24 and 25 . These data represent averages of results obtained in two tests which were highly repeatable. The 
measured signal were periodic indicating that the reaction rate in the pulse combustor is periodic. The measured data were analyzed with a FFT software to obtain the amplitudes and phases (with respect to the local pressure oscillations) of the radiation signals. Under this operating condition, the combustor pulsations were dominated by the first harmonic of the combustor tube which whose frequency and pressure amplitude equaled $218 \mathrm{~Hz}$ and $166 \mathrm{~dB}$, respectively.

An examination of the data in Fig. 24 indicates that the amplitude of the C$\mathrm{H}$ radiation along the centerline of the injection system increases from nearly zero along an initial distance of $2 \mathrm{D}$, where $\mathrm{D}$ is the injection port diameter, to a maximum value at a distance of $7 D$ downstream of the injection point. After reaching a maximum value at $Y=7 D$, the amplitude of the radiation decreases as $Y$ further increases. On the other hand, the amplitudes of the radiation in the . vicinity of the side walls of the injection duct are always low. These results indicate that combustion begins at a distance of about $3 \mathrm{D}$ from the injection port and that the highest heat release rate occurs in a region between approximately $3 \mathrm{D}$ and $7 \mathrm{D}$; that is, in a region located between 2.1 and 4.9 inches downstream of the injector.

Figure 25 presents phase differences between the local radiation and pressure oscillations measured in the above discussed tests. Since the magnitudes of the radiation signals measured close to the injection point were in the range of the background noise, the phases of these signals (i.e., the data in the first row of Fig. 25) are not meaningful. An examination of the phase difference data in Fig. 25 indicates that the phase difference between the radiation and pressure oscillations along the injection duct centerline increases monotonically from -56.3 to +60.3 as $Y$ increases. Examination of the phase variations along the side walls of the injection duct shows that the phase there decreases as $Y$ increases when $2 D<Y<5 D$, reaching a minimum value at $Y=5 D$, and it increases thereafter. These trends suggest that combustion may first occur near the walls in the vicinity of the $\mathrm{Y}=5 \mathrm{D}$ location and it propagate from there simultaneously upstream and downstream towards the injection port and the combustor, respectively. 


\section{Task IV: Visualization Studies}

\section{Schlieren Method Tests}

A Schlieren system was set up to take high speed Schlieren movies of the reacting flow field, see Fig. 26 . By recording the density variations in the reacting flow field, the high speed Schlieren movies provide descriptions of the main features of the reacting flow field and their time evolution.

At the outset of these efforts, it was expected that the Schlieren movies would reveal the presence of vortex structures similar to those that characterized the radiation patterns measured by the imaging system. It was also expected that the high speed Schlieren movies would provide better descriptions of the temporal variations of these vortical structures. However, the classical Schlieren setup failed to produce satisfactory images of the reacting flow field, because the intense heat transfer from the combustion process caused the quartz windows to deform, unfocusing the Schlieren beam.

To resolve this problem, an attempt was made to cool the windows with flowing nitrogen films. These films were expected to increase the resistance to convective heating of the windows by the hot gases while simultaneously cooling the hot windows. The cooling films were injected via a new $.5 \mathrm{~mm}$ slots in drilled in a new injector mounting plate, see Fig. 27. Room temperature nitrogen was chosen because it is inert and was not expected to affect the reaction process.

Unfortunately, the nitrogen cooling failed to produce satisfactory results. With low nitrogen injection rates, which produced film velocities below 30 $\mathrm{m} / \mathrm{sec}$, the cooling was inadequate and no significant reduction in window deformation was observed. When the nitrogen flow rate was increased to produce film velocities of $100 \mathrm{~m} / \mathrm{sec}$, the deformation of the quartz glass was significantly reduced. However, when the film velocities increased to these magnitudes, the nitrogen flow apparently interfered with the combustion processes inside the injection duct, resulting in unstable pulsations with constantly varying amplitudes and frequencies.

Another effort was made to resolve this problem. A modified Schlieren, which attempted to restore the fidelity of the images by optically "subtracting" the image distortion caused by the windows 2,3 , was developed. Tests using the modified Schlieren system were performed for a large number of different combustor operation conditions. A conventional high speed movie camera and a 
high speed video motion analyzer with a short exposure time were used to record the Schlieren images. The resulting images showed the presence of a reaction zone similar to that of a turbulent flame and the absence of any periodic phenomena, such as vortex structures. These observations are in contrast with the radiation intensity measurements that strongly suggested the periodic formation of reacting vortices within the combustion region played a key role in the driving of the pulsations.

\section{Flow Visualization with a High Speed Video System}

Another effort to visualize the flow field and combustion process was made by directly recording images of the combustion zone with a high speed video camera. The high speed video camera utilized in this study was a Kodak Ektapro EM Motion Analyzer, see Fig. 28. The system consists of an intensified imager, an intensified imager controller, a processor, a video cassette recorder and a video monitor. The Kodak EktaPro Intensified Imager has a spatial resolution of $192 \times 239$ pixels and a spectral response from $440 \mathrm{~nm}$ to $700 \mathrm{~nm}$. It can be operated at speeds ranging from 30 to 6000 frames per second and exposure times of 10 microseconds to 5 milliseconds. The processor, Kodak EktaPro EM Process Model 1012, can store 1000 full frame images and up to 12,000 split frame images. Images obtained by the intensified imager are first stored in the processor and then played back at a desired rate. The playback rate can vary from 0 to 960 frames per second. A video monitor, Kodak Ektapro 1000 Monitor, was used to display the video images and a video cassette recorder was used to down load the images to a video tape for further analysis.

The video images obtained in these tests were similar to those obtained by the imaging system earlier under this program. In order to obtain more information about the flow field, images were obtained by seeding the flow field with aluminum oxide particles 2,3 . The objective of this method was to obtain a description of the flow field by tracking the radiation from the heated particles. Images obtained by this method suggested that two vortex-like structures are formed early in the cycle. These structures expand as they are convected downstream until they merge and occupy most of the flow region. Simultaneously, the intensity of the radiation from the flow increases in magnitude and it reaches a maximum value as the structures coalesce. After that, the structures decrease in size and their radiation intensity diminishes. 


\section{Driving Mechanism Studies}

\section{Existence of Traveling Waves in the Air/Fuel Feed Lines}

This study investigated the possibility that pressure oscillations inside the air and fuel feed line produced periodic air and fuel supply rates into the combustor that were responsible for the formation of a periodic heat release process that drove the combustor pulsations. Acoustic pressure measurements revealed the presence of traveling pressure waves inside the air and fuel feed lines. Since the fuel and air velocities inside the feed lines were subsonic, the combustor pressure oscillation produced disturbance that propagated with the speed of sound in the upstream direction along the fuel and air feed lines. To investigate this phenomenon, the measured pressures were Fourier analyzed to obtain their amplitudes, frequencies and the phases. In the following, some of the results are briefly discussed in an effort to explain how these traveling pressure waves contribute to the driving of the oscillations in the developed combustor; additional results are presented in Refs. 2 and 3.

Figure 29 presents data obtained in a test in which the fundamental acoustic mode of the combustor tube was excited. It shows that the amplitudes of the traveling pressure wave decayed with distance away from the opening of the 0.75 inch inside diameter air feed line. Furthermore, the phase of the traveling waves increased linearly with the distance from the air injection point. The data obtained when first harmonic of the combustor was excited is presented in Fig. 30. The trend is similar to that exhibited in the fundamental mode case. Similar data measured in fuel feed line are presented in Figs. 31 and 32. The observation that the phase varies linearly with distance along the feed lines suggests that the measured oscillations are traveling pressure waves that propagate at a constant speed inside both the air and fuel feed lines. Calculations of the speeds of sound in air and propane and the average velocities (based upon measured volume flow rates and cross-sectional areas of the feed lines) have supported the above conclusion. The predicted traveling wave propagation speeds were in excellent agreement with the experimental data.

\section{Air and Fuel Injection Rate Modulation and Periodic Combustion}

The changes in the combustor pressure were transmitted upstream into the air and fuel feed lines as traveling waves through the fuel and air injection ports. Hence, the fluctuating components of the particle velocities near the fuel and air 
injector were directed into the combustor when its pressure was below average and away from the combustor when its pressure was above average. Using calculated impedances of air and propane and measured acoustic pressure amplitudes, the acoustic velocities in the fuel and air lines were calculated. Figure 33 shows the dependencies of the acoustic velocity amplitudes in air and propane upon the amplitude of pressure oscillation in $\mathrm{dB}$. It shows that when the amplitude of the pressure oscillation is around $170 \mathrm{~dB}$, the amplitudes of the acoustic velocity oscillations in the propane and air lines are $19 \mathrm{~m} / \mathrm{sec}$ and 23 $\mathrm{m} / \mathrm{sec}$, respectively.

By superposition of the acoustic velocities upon the mean flow velocities the fluctuating fuel and air feed rates into the combustor were calculated. They showed that fuel and air injection rates into the combustor varied periodically during a cycle. The relative amounts of fuel and air flow rates injected into the combustor during the first (i.e., when the combustor pressure was "positive") and second (when the combustor pressure was "negative") halves of the pressure oscillation cycle were determined by the ratio of the amplitudes of acoustic velocities to the mean injection velocities of the air and fuel. Some of the calculated results are shown in Fig. 34. This figure indicates that as the ratio of the acoustic velocity to the mean injection velocity increases, the relative amounts of air and fuel injected during the "positive" and "negative" half cycles of the oscillations decrease and increase, respectively. For example, when the amplitude of the acoustic velocity equals to the mean injection velocity, more than $82 \%$ of the total air or fuel is injected into the combustor in the "negative" half of the pressure oscillation cycle.

In summary, traveling pressure waves excited inside the air and fuel feed lines induce fluctuations of the fuel and air injection velocities, which, in turn, produce oscillatory propane and air injection rates. As a result, the majority of the fuel is always injected into the combustor in the "negative" half of the cycle. This periodic variation of the fuel injection results in a periodic variations of the combustion region air/fuel ratio, the reaction and heat release rates. However, whether this periodic heat release process can drive combustor pressure oscillation depends upon the phase difference between the heat release and pressure oscillations. According to Rayleigh's criterion, driving of pressure oscillations occurs when the magnitude of this phase is smaller than ninety degrees. 


\section{Driving Due to Air and Fuel Injection Rates Modulation}

As mentioned previously, the phase of the maximum heat release occurs during the phase when the "maximum" injected amount of fuel is burned. This "Excess" fuel is always injected into the combustor during half the cycle when the acoustic pressure is negative. The time when the "excess" fuel energy is released relative to the pressure oscillation depends upon the delay time between fuel and air injection and the instant of energy release. For the developed premixed type injection system, the characteristics of this delay time are largely determined by the characteristics of the chemical time, which is dependent upon the air/fuel ratio. Consequently, the air/fuel ratio when the "excess" fuel is injected into the combustor will determine the phase of the maximum heat release and, thus, determine if driving will occur. Driving occurs when this maximum heat release is in phase with the maximum pressure amplitude. The excited pressure oscillation affects the air and fuel injection rates which, in turn, controls the air/fuel ratio modulation and, thus, the phase of maximum heat release, which completes the feedback loop.

\section{Investigation of the Mode-Shifting Phenomenon}

It was found that, when the premixed injector is used, fundamental modes oscillations are excited in the combustor at low air/fuel ratios and they are replaced by first harmonic oscillations as the air/fuel ratio $\alpha$ is increased, see Fig. 35 .

The excitation of different mode oscillations at different values of $\alpha$ with the premixed type injector may be explained by considering the characteristic times that control the combustion process and the characteristics of the excited combustor modes. According to Rayleigh's criterion, an unsteady heat release process will drive a given combustor mode if it has a component in phase with the mode's pressure oscillation, and if the heat is released in a region of the combustor where the pressure amplitude of this mode is significant. It can be also shown qualitatively that in order to drive a given mode, the combustion time, which depends upon the convection, mixing and reaction times, must approximately equal half the period of the oscillation of the excited mode. Thus, a given combustion process will drive those modes for which the above stated conditions are satisfied. The observation that the pulse combustor operates at its fundamental and first harmonic modes at fuel rich and fuel lean conditions, 
respectively, strongly suggests that the characteristic combustion time decreased as the value of $\alpha$ increased.

The above described mode shifting can be explained by the following conjecture. In the reported tests, $\alpha$ was increased by increasing the air flow rate. This, in turn, increased the velocity of the reactants entering the injection duct, which reduced the time required for the reactants to reach the combustion region. In addition, the high flow velocities increased mixing rates between fresh reactants, combustion products and reacting pockets left over from the previous cycle. These effects are expected to decrease the characteristic combustion time. As the combustion time decreased, its magnitude became considerably smaller than half the period of the fundamental combustor mode and it approached the magnitude of half the period of the first harmonic of the combustor. Since the "above stated time condition requires that the combustion time approximately equal half the period of the oscillation, the first harmonic of the combustor was excited as the air/fuel increased because of the decrease in the combustion time produced by the increase in the air/fuel ratio. This conjecture is schematically described in Figure 36. This conjecture was supported by calculations of the characteristic times for two test conditions in which the fundamental and first harmonic modes were excited 2,3 . The characteristic time calculated for each of these test conditions approximately equaled half the period of the excited oscillations.

\section{The Role of Traveling Waves in the Reactants Feed Lines.}

Modulation of the air and fuel injection rates into the combustor and vortex shedding were identified as two important components of the driving mechanisms. It was not clear, however, whether these processes depend upon one another or whether they can drive pulsations independently. To resolve this question, tests were conducted in which the modulation of the air and/or fuel injection rates was eliminated by installing sonic orifices in the air and/or fuel feed lines, see Figure 37(a). It was, thus, possible to determine whether pulse combustion operation is possible in the absence of such modulations. If this was the case, then it would follow that the pulsations could be driven by some other mechanisms, such as vortex shedding. Furthermore, the effect of modulations in the reactants flow rates upon the combustor performance was investigated by comparing the combustor performance in the presence and absence of such modulations. 
This study consisted of four series of tests in which different combustor configurations were utilized, see Table 1. In each of these tests one or both reactant injection rates were choked by use of sonic orifices, and the choking of the line was verified by pressure measurements that showed that no disturbances were present in the feed line upstream of the orifice.

The first series of tests investigated the effect of choking the fuel line with a sonic orifice upon the combustor performance. These tests investigated the dependence of the amplitudes of the excited combustor modes upon the nondimensional air/fuel ratio at a fixed propane input rate of 1.5 SCFM. The effect of choking the fuel line was determined by comparing the resulting combustor performance with its performance under normal operating conditions. This comparison showed that choking the fuel lines significantly changed the combustor performance, see Figures 38 through 41 . In the absence of fuel line choking, both the fundamental and first harmonic longitudinal modes of the combustor were excited with the former attaining its maximum amplitude at $\alpha=1.5$ and the latter at $\alpha=1$. When sonic orifices were installed in the fuel lines, the maximum amplitude of the fundamental and first harmonic of the longitudinal modes of the combustor were excited at $\alpha=0.7-0.8$ and $\alpha=1.0-1.1$, respectively, and pulse combustor operation was not attained beyond $\alpha=1.45$.

The second series of tests investigated the effect of choking the air line and choking both the fuel and the air lines upon the combustor performance. Table 2 summarizes the results of these tests. It was found that when only the air feed line was choked, unstable pulse combustion operation was possible in a very narrow range of $\alpha$. However, when both the air and fuel lines were choked, pulse combustion operation was not possible.

In the third series of tests, the performance of the combustor was studied by moving the sonic orifice in the air feed line 12 inches upstream of its original position. This modification changed the acoustic characteristics of the air supply system and the performance of the pulse combustor. The results of these tests are presented in Figs. 42 and 43. They show that when both the air and fuel sonic orifices were installed at the end of the feed lines, pulsations were not excited. However, when the sonic orifice was installed 12 inches upstream of its original position, the fundamental and first harmonic modes of the combustor were excited at various ranges of $\alpha$.

In the last series of tests, an attempt was made to eliminate the air and fuel flow rate modulations without significantly changing the flow field in the 
its original position, the fundamental and first harmonic modes of the combustor were excited at various ranges of $\alpha$.

In the last series of tests, an attempt was made to eliminate the air and fuel flow rate modulations without significantly changing the flow field in the injection duct. When the air line was choked, the 0.28 inches diameter sonic orifice was installed approximately 1.5 inches above the mixture injection orifice whose diameter equaled 0.7 inches, see Figure 37(a). Therefore, the sonic air jet could pass through the mixture injection orifice undisturbed. In the modified design, an impingement plate was installed in the reactants mixing chamber 0.35 inches downstream of the air line orifice, see Figure 37(b). The impingement plate consisted of a round metal sheet of 0.4 inches in diameter and it was attached to the air injector by three legs. In this configuration the sonic air jet was forced to impinge upon the plate before mixing with the fuel, which should have prevented the air jet from passing through the mixture injection orifice undisturbed. Four comparable tests were conducted, and the configuration of Figure 37(b) was used in two of these tests. The results are presented in Table 3 and Figures 44 through 47 . A comparison of these results with the observations from Test No. 3 in Table 2 reveals that the impingement plate significantly changed the combustor performance. When the plate was absent and the air line was choked, no stable pulsations were obtained, see Test No. 3 in Table 2. In contrast, when the plate was installed, high amplitude stable pulsations were obtained for both the fundamental and first harmonic modes, see Figure 46. These results indicate that the characteristics of the flow fields in the mixing chamber and in the injection duct play an important role in the driving of the pulsations. Finally, it should be noted, see Figure 47 and Table 3, that no pulsations were excited when both the air and fuel lines were choked, even when impingement plate was present in the injection system, indicating that the air and fuel feed rates modulations are an essential element of the driving mechanisms.

\section{Laser Donpler Velocity Measurements}

A limited number of laser Doppler velocimeter measurements were carried out in the tangential injection duct in order to gain additional insight into the flow field in that part of the pulse combustor. These measurements were carried out because the radiation images shown in Figs. 16-22 strongly suggest 
that the processes that occur in the tangential injection duct are responsible for driving the pulsations in the pulse combustor.

Two components of velocity were measured simultaneously using an Aerometrics, two channel, phase Doppler anemometer powered by a seven watt argon ion laser. This velocimeter includes a pair of Bragg cells that permit the detection of back flow. This is critical in this application. The signal processor of the system uses an FFT routine to determine velocities and their distributions. Aluminum Oxide particles were used to seed the flow.

Velocity distributions in the injection duct in selected planes parallel to the injection plate (see Fig. 2) were measured in cold flow as well as in flow with combustion. The reactants flow rate was kept constant at 47.9 SCFM during these runs. Velocities measured at planes located 2.5 and 3.5 inches downstream of the fuel/air injector are shown in Figs. 48 and 49 . In both cases, the flow is dominated by large, downward injection velocity at the center of the duct. At the same time the presence of significant back flow near the wall of the duct indicates the that a large toroidal vortex has been formed around the central jet. This type of flow field is commonly found in sudden expansion dump combustors.

Comparisons of the hot and cold flow data in Figs. 48 and 49 indicate that the pulse combustion process slightly increases the magnitude of both the incoming jet velocity of the reverse flow. In addition, it was observed that presence of combustion reduced the level of turbulence in the flow. A similar observation had been made in measurements behind a backward facing step as part of a different investigation. Given the limited resources, it was not possible to obtain enough velocity data to determine whether vortex shedding occured during any phase of the cycle. 


\section{PUBLICATIONS}

1. "Performance of a gas buming Rijke type pulse combustor with tangential air/fuel injection," Proceedings of the International Symposium on Pulsating Combustion, Monterey, 1991. Also accepted for publication in Combustion Science and Technology, 1992.

2. "Vortex shedding and periodic combustion processes in a Rijke type pulse combustor," Proceedings of the International Symposium on Pulsating Combustion, Monterey, 1991. Also accepted for publication in Combustion Science and Technology, 1992.

3. "Combustion of heavy liquid fuels in a Rijke type pulse combustor," Published in ASME publication Combustion Instabilities Driven by Thermo-Chemical Acoustic Sources, edited by A. S. Hersh, I. Catton and R. F. Keltie. NCA-Vol. 4 / HTD-Vol. 128.

4. "Flame stabilization and combustion of heavy liquid fuels in a Rijke type pulse combustor," Published in ASME publication Fossil Fuel Combustion. 1991. edited by R. Ruiz, PD-Vol. 33.

5. "Combustion of heavy fuel oils in a Rijke type pulse combustor with tangential air and fuel injection," Proceedings of IUTAM Symposium on Aerothermo-dynamics in Combustion, Taipai, 1991. 


\section{REFERENCES}

1. Zinn, B. T., Daniel, B. R., Shani, S., Bai, T. and Zhang, Z., "Investigation of the Characteristics of Liquid Fuel Burning, Rijke Type, Pulse Combustors," Final Report for DOE Contract No. DE-AS04-85AL31881, February, 1989.

2. Zinn, B. T., Jagoda, J. I., Daniel, B. R., Bai, T. and Cheng, X. C., "Investigation of the Mechanism in Rijke Pulse Combustors with Tangential Air and Fuel Injection," Annual Report for DOE Contract No. DE-AS04-85AL31881, September, 1990.

3. Zinn, B. T., Jagoda, J. I., Daniel, B. R., Bai, T. and Cheng, X. C., "Investigation of the Mechanism in Rijke Pulse Combustors with Tangential Air and Fuel Injection," Annual Report for DOE Contract No. DE-AS04-85AL31881, September, 1991. 
Table 1 - Summary of the Test Program

\begin{tabular}{|c|c|c|c|c|}
\hline $\begin{array}{c}\text { Test } \\
\text { Sequence }\end{array}$ & $\begin{array}{l}\text { Fuel Flow } \\
\text { Rate SCFM }\end{array}$ & $\begin{array}{l}\text { Air Feed } \\
\text { Line }\end{array}$ & $\begin{array}{l}\text { Fuel Feed } \\
\text { Line }\end{array}$ & References \\
\hline \multirow{2}{*}{1} & \multirow{2}{*}{1.5} & 0 & 0 & Figures 41,42 \\
\hline & & 0 & 1 & Figures 43,44 \\
\hline \multirow{4}{*}{2} & \multirow{4}{*}{$\begin{array}{l}1.1- \\
1.17\end{array}$} & 0 & 0 & \multirow{4}{*}{ Table 2} \\
\hline & & 0 & 1 & \\
\hline & & 1 & 0 & \\
\hline & & 1 & 1 & \\
\hline \multirow{2}{*}{3} & \multirow{2}{*}{$\begin{array}{l}1.2- \\
1.17\end{array}$} & 1 & 1 & Figure 45 \\
\hline & & 2 & 1 & Figure 46 \\
\hline \multirow{4}{*}{4} & \multirow{4}{*}{1.1} & 0 & 0 & Figure 47 \\
\hline & & 0 & 1 & Figure 48 \\
\hline & & 3 & 0 & Figure 49 \\
\hline & & 3 & 1 & Figure 50 \\
\hline
\end{tabular}

0 -..- Regular configuration

1 -..- Sonic orifice installed at the line outlet

2 --- Sonic orifice installed 12 inches upstream of the line outlet

3 -.-- Sonic orifice with impingement plate installed at the line outlet 


\section{Table 2 - Summary of Test Sequence No. 2}

Fuel: Propane

Fuel Flow Rate: 1.11-1.17 SCFM

\begin{tabular}{|c|c|c|l|}
\hline $\begin{array}{c}\text { Test } \\
\text { No. }\end{array}$ & Air Line & Fuel Line & \multicolumn{1}{|c|}{ Observations } \\
\hline 1 & 0 & 0 & $\begin{array}{l}\text { Stable fundamental and harmonic } \\
\text { pulsations. Fund. mode not excited under } \\
\text { fuel rich conditions. }\end{array}$ \\
\hline 2 & 0 & 1 & $\begin{array}{l}\text { Stable fundamental and harmonic } \\
\text { pulsations. Fund. mode readily excited } \\
\text { under fuel rich conditions. }\end{array}$ \\
\hline 3 & 1 & 0 & $\begin{array}{l}\text { Pulsations could only be obtained within } \\
\text { an extremely narrow } \alpha \text { range and were } \\
\text { very unstable. }\end{array}$ \\
\hline 4 & 1 & 1 & $\begin{array}{l}\text { No pulsations could be excited over a wide } \\
\text { range of combustor operating conditions. }\end{array}$ \\
\hline
\end{tabular}

0 --- Regular Orifice Installed at the Line Outlet.

1 -..- Sonic Orifice Installed at the Line Outlet. 
Table 3 - Summary of Test Sequence No. 4

Fuel: Propane

Fuel Flow Rate: 1.1 SCFM

\begin{tabular}{|c|c|c|l|}
\hline $\begin{array}{c}\text { Test } \\
\text { No. }\end{array}$ & Air Line & Fuel Line & \multicolumn{1}{|c|}{ Observations } \\
\hline $\mathbf{1}$ & $\mathbf{0}$ & $\mathbf{0}$ & $\begin{array}{l}\text { Stable fundamental and first harmonic } \\
\text { pulsations. Fundamental mode at fuel rich } \\
\text { was not excited, see Figure 47. }\end{array}$ \\
\hline $\mathbf{3}$ & $\mathbf{0}$ & $\mathbf{1}$ & $\begin{array}{l}\text { Stable fundamental and first harmonic } \\
\text { pulsations. Fund. mode at fuel rich was } \\
\text { easy to excite, see Figure 48. }\end{array}$ \\
\hline $\mathbf{3}$ & $\mathbf{3}$ & $\mathbf{0}$ & $\begin{array}{l}\text { High amplitude stable pulsations can be } \\
\text { obtained for both the fundamental and } \\
\text { harmonic modes, see Figure 49. }\end{array}$ \\
\hline
\end{tabular}

0 ---- Regular Orifice Installed at the Line Outlet.

1 -..- Sonic Orifice Installed at the Line Outlet.

3 -... Sonic Orifice with the Impingement Plate Installed at the Line Outlet. 


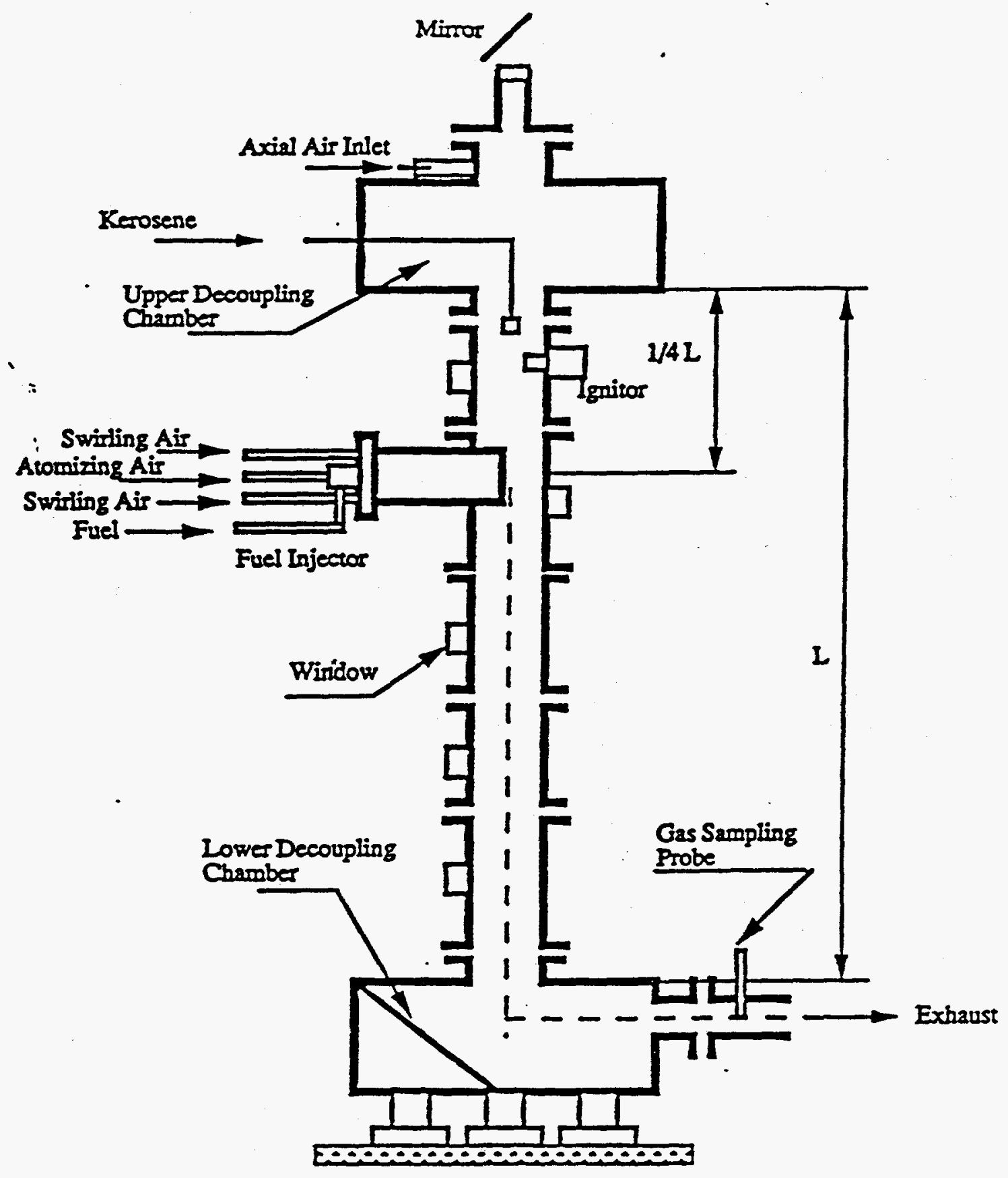

Fig. 1 A Schematic of the Developed Liquid Fuel Burning Rijke Type Pulse Combustor with Tangential Fuel and $A$ ir Inlection System. 


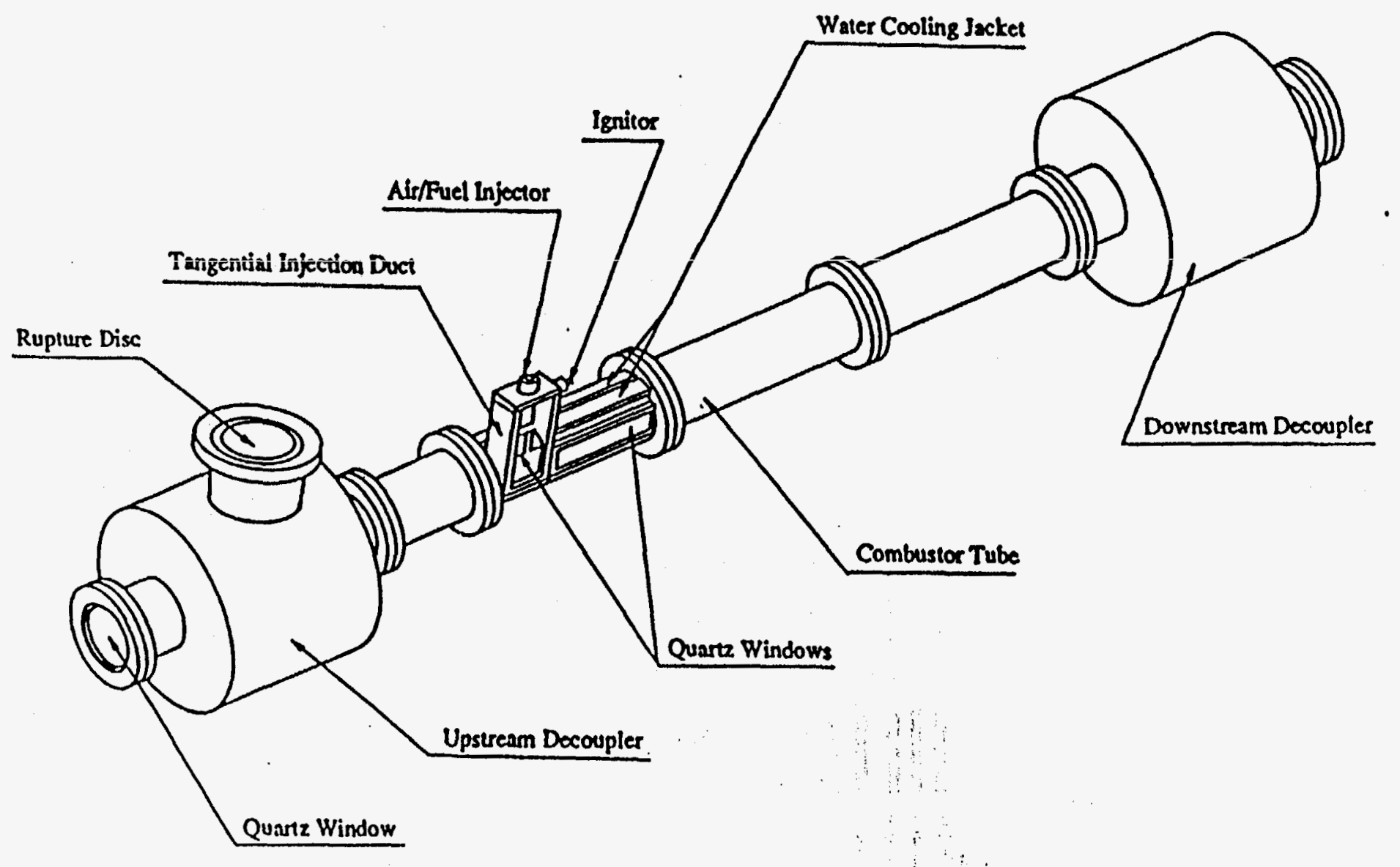

Fig. 2 A Schematic of the Developed Experimental Setup which Utilizes a Tangential Air/Fuel Injection System Equipped with Quartz Windows. 


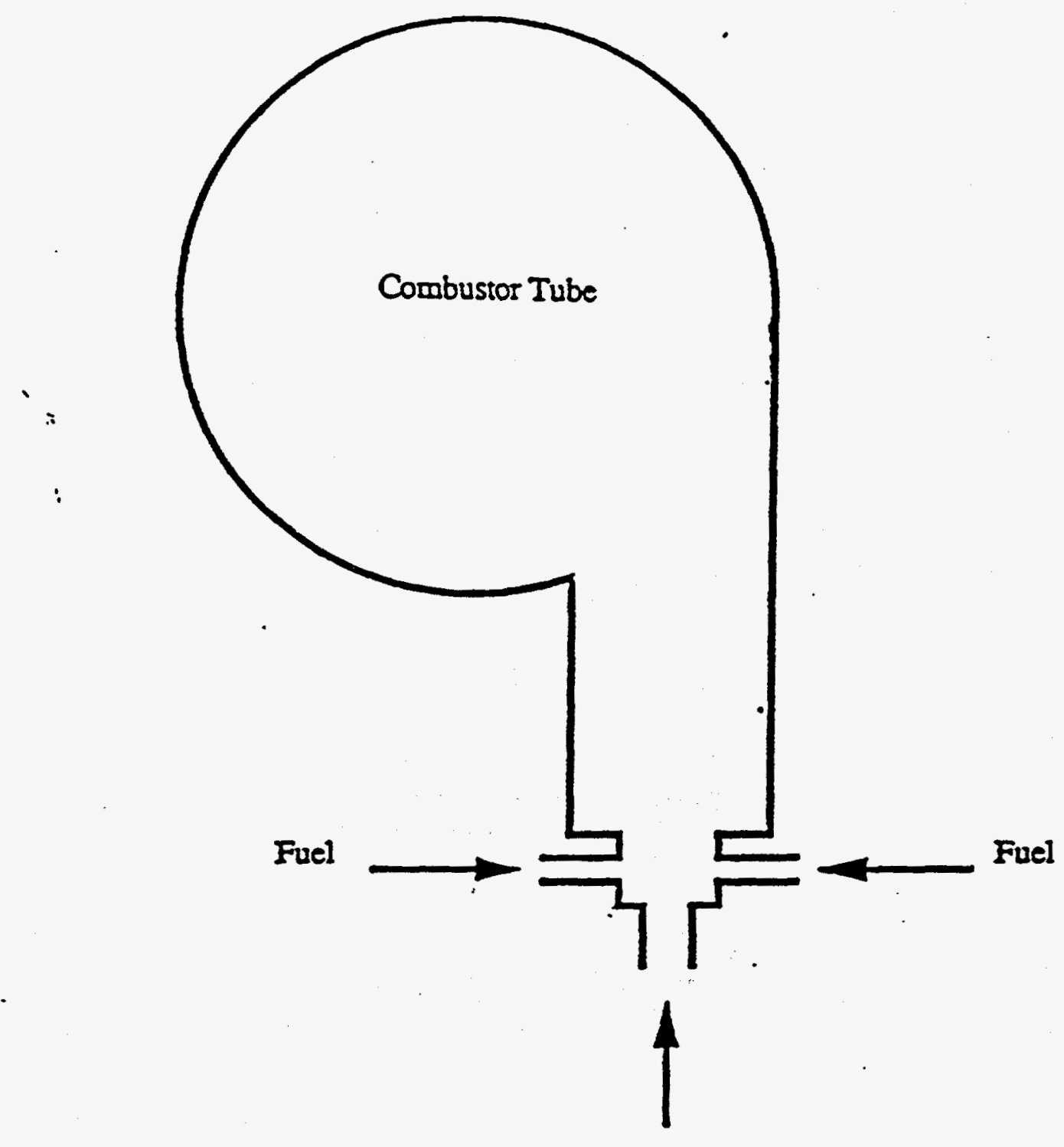

Air

Fig. 3 A Schematic of a Premixed Tangential Injection System. 


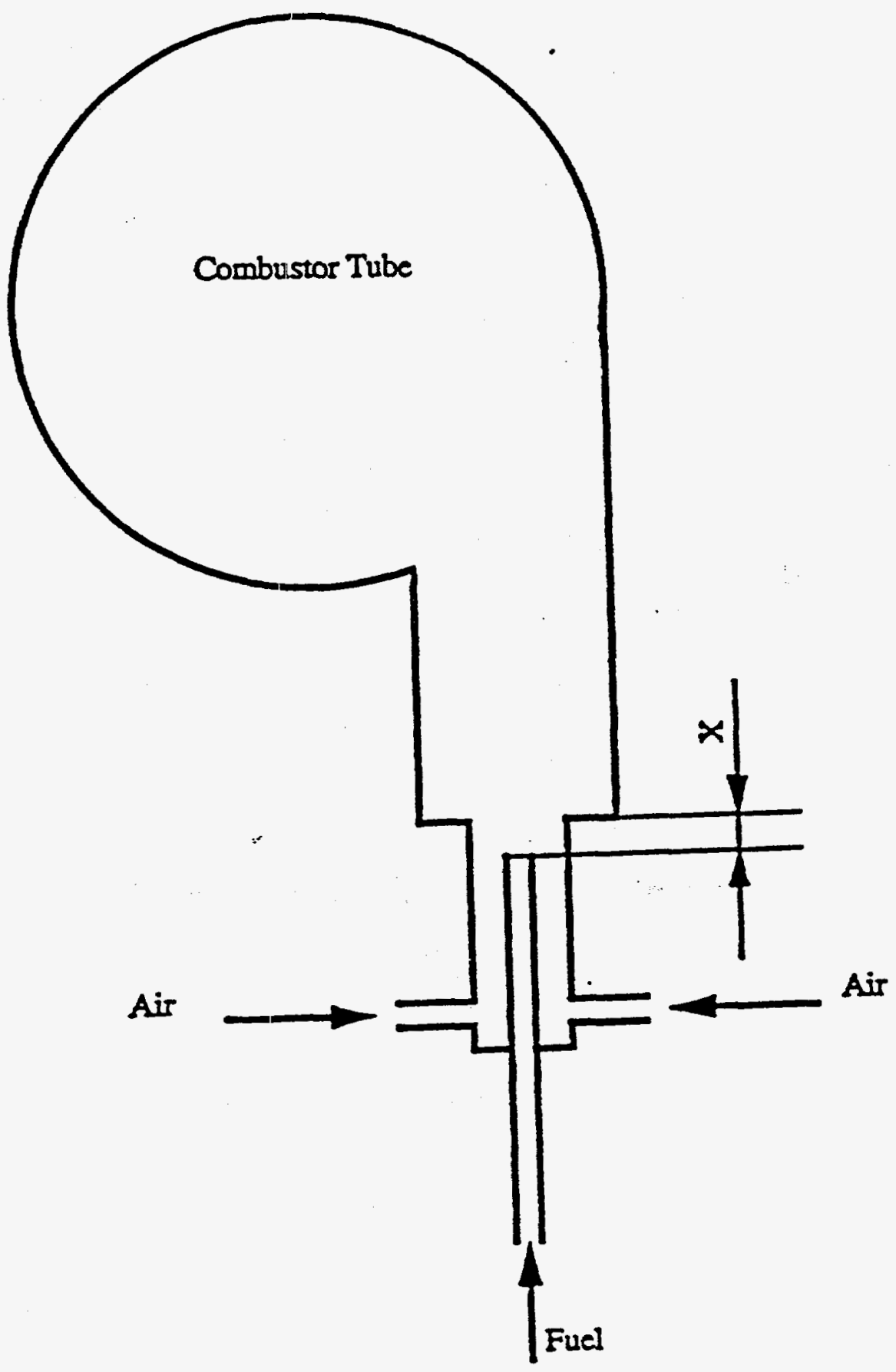

Fig. 4 A Schematic of a Diffusion Tangential Injection System. 


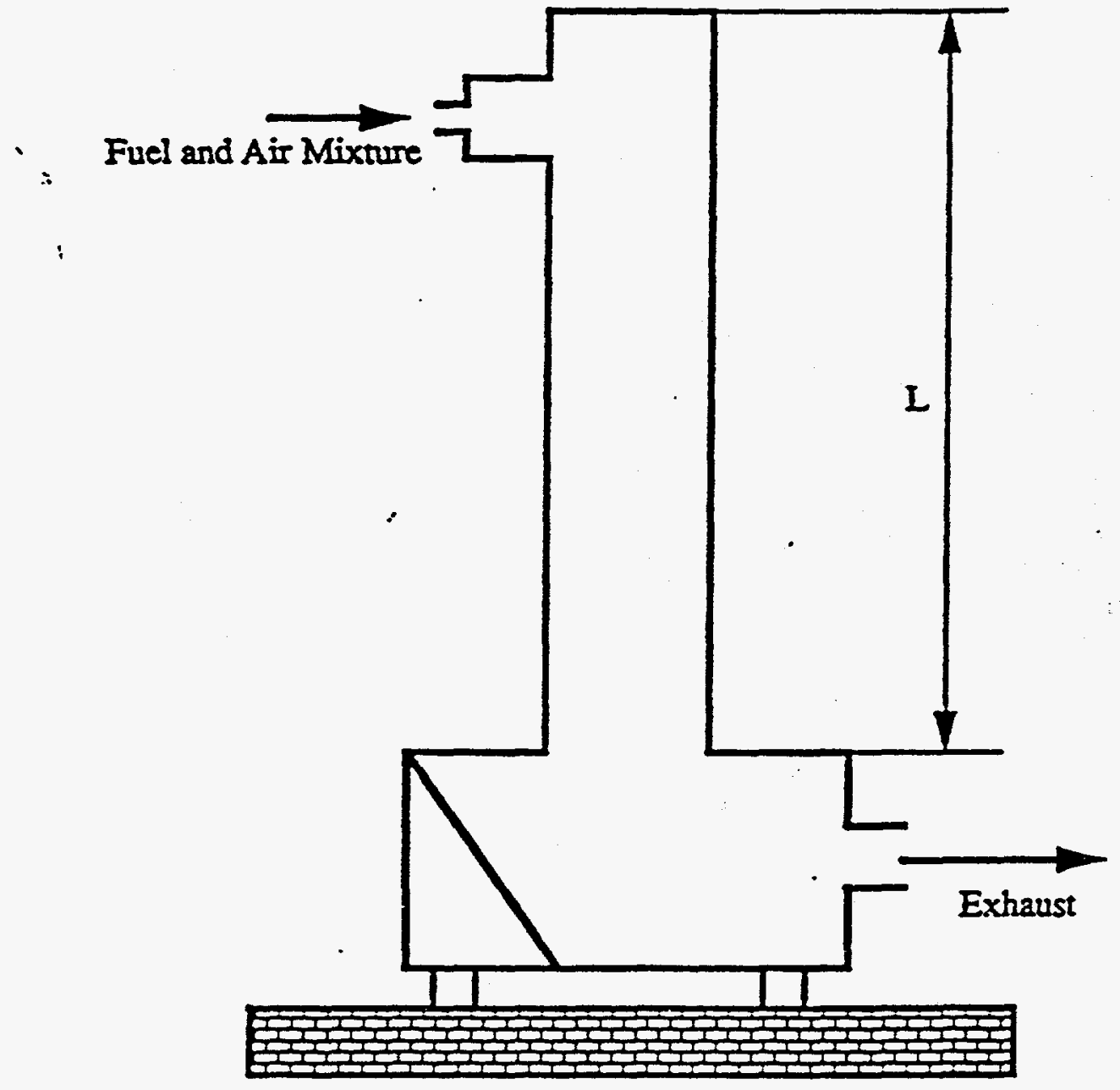

Fig. 5 A Schematic of the Pulse Combustor Setup with a Closed Upper End. 


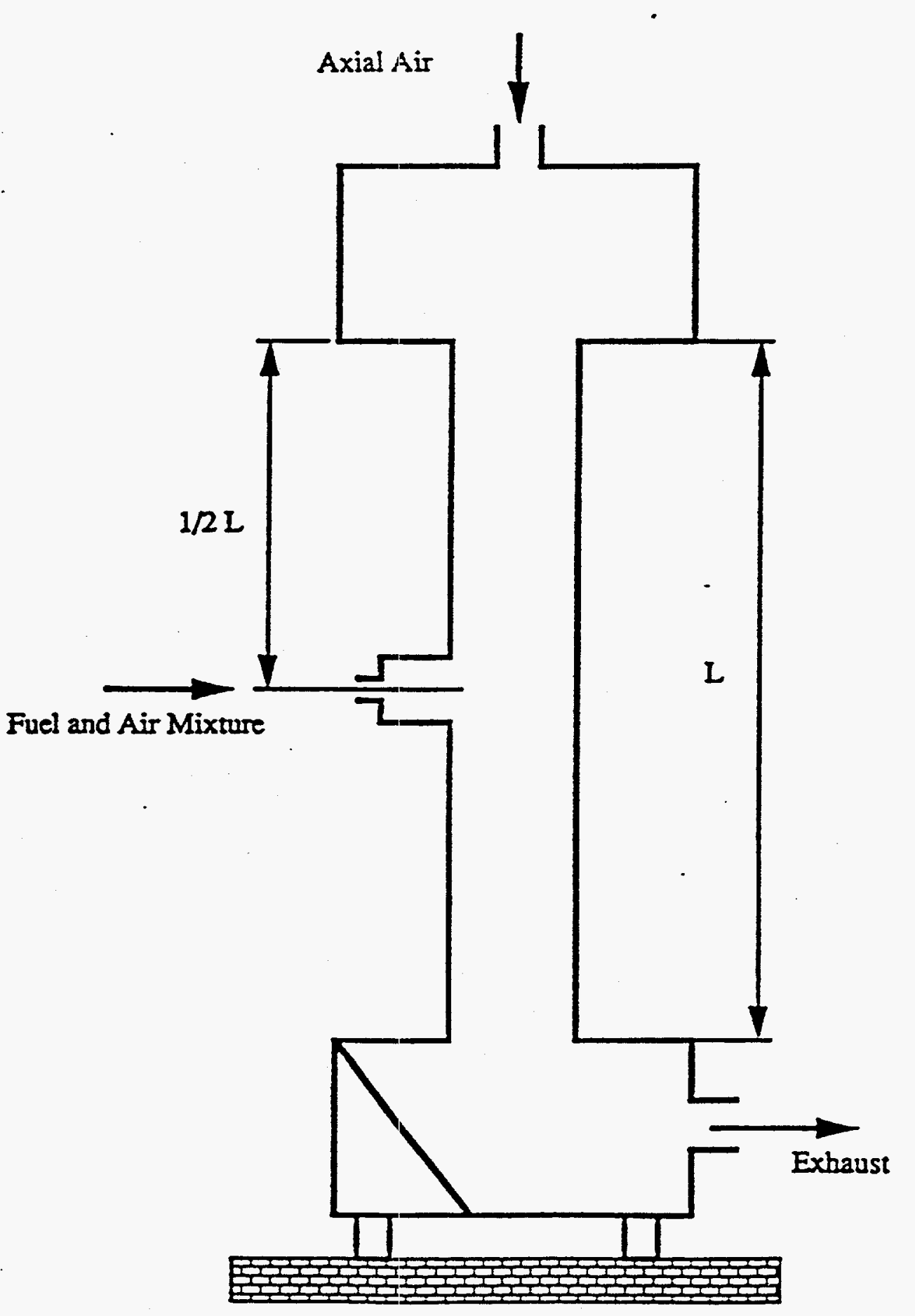

Fig. 6 A Schematic of the Pulse Combustor Setup with Fuel Injected at the $1 / 2$ L Location. 


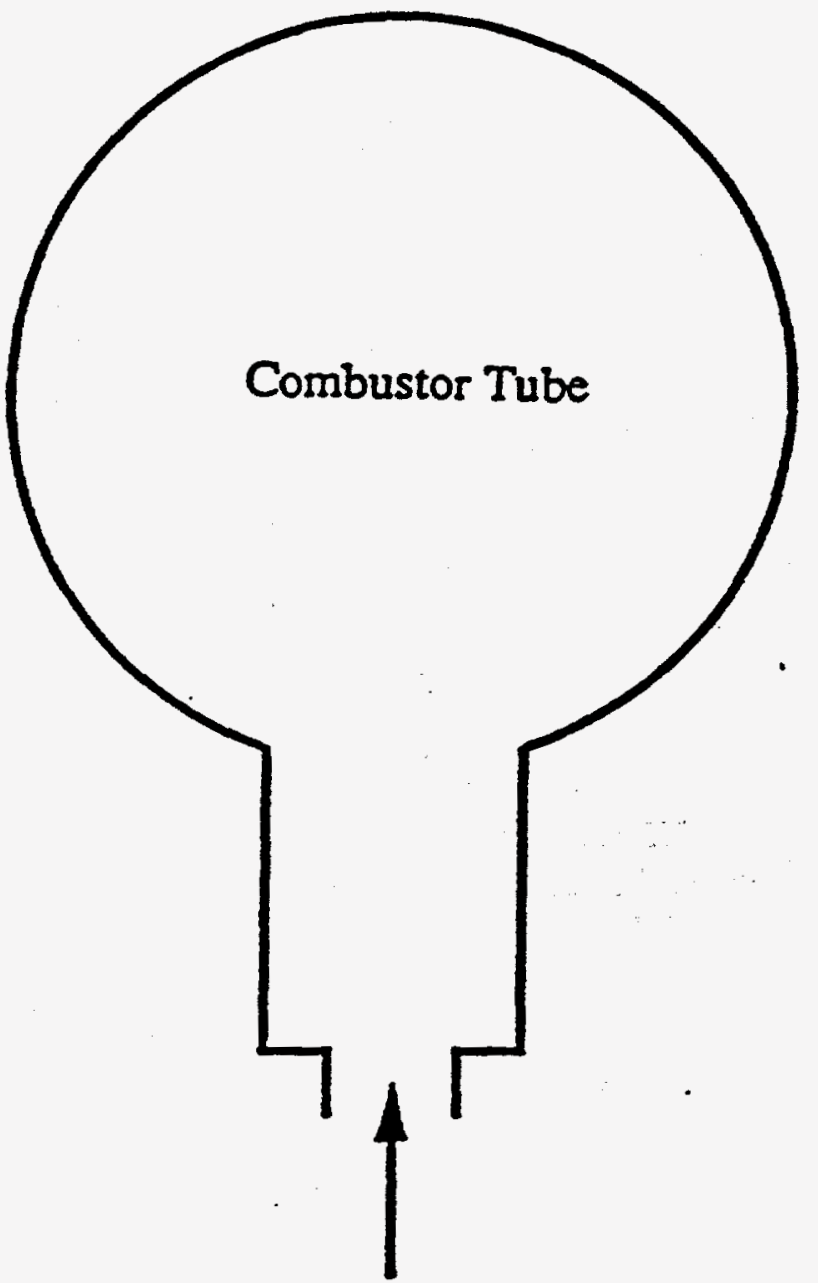

Fuel and Air Mixture

Fig. 7 . A Schematic of the Radial Injection Scheme. 


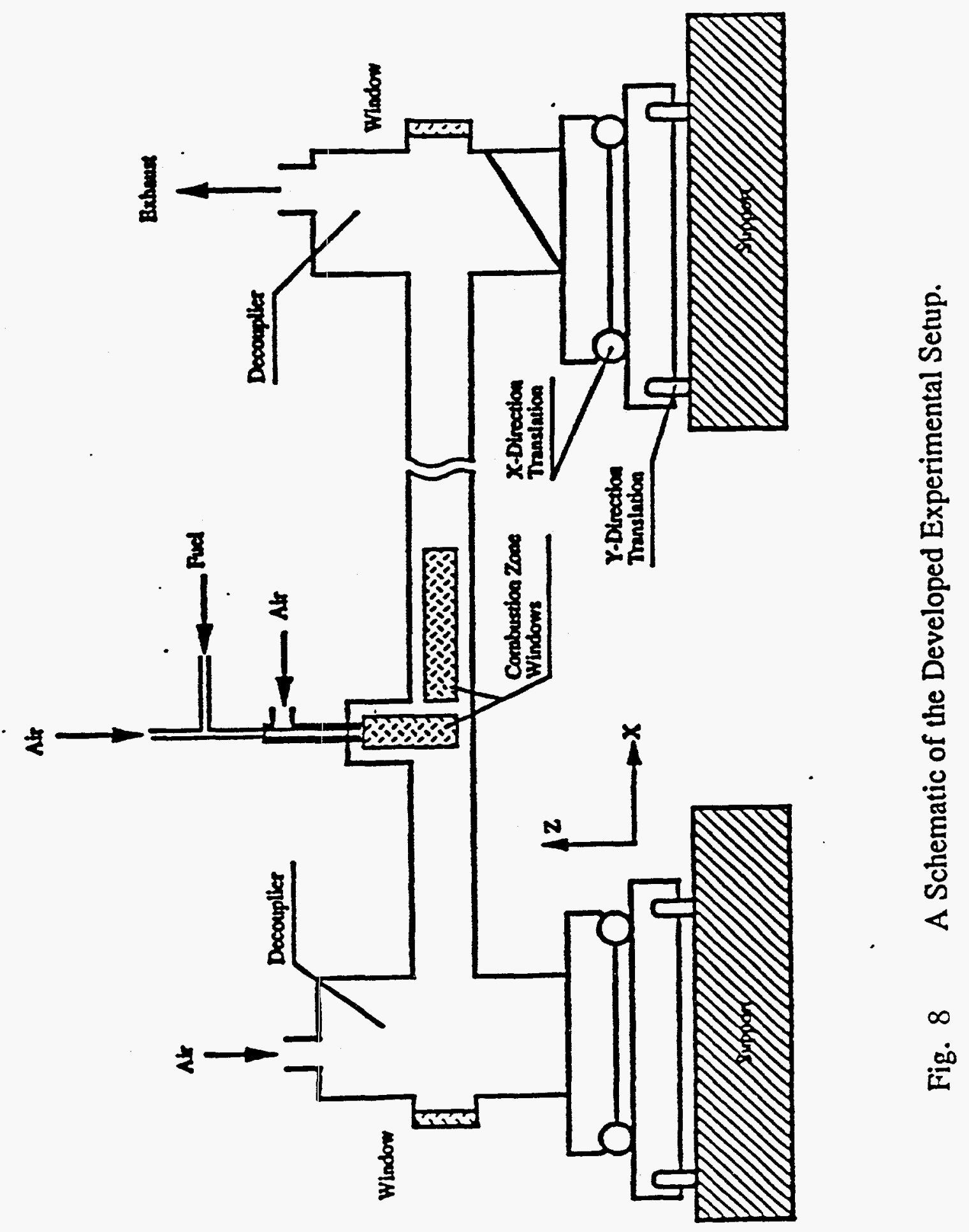




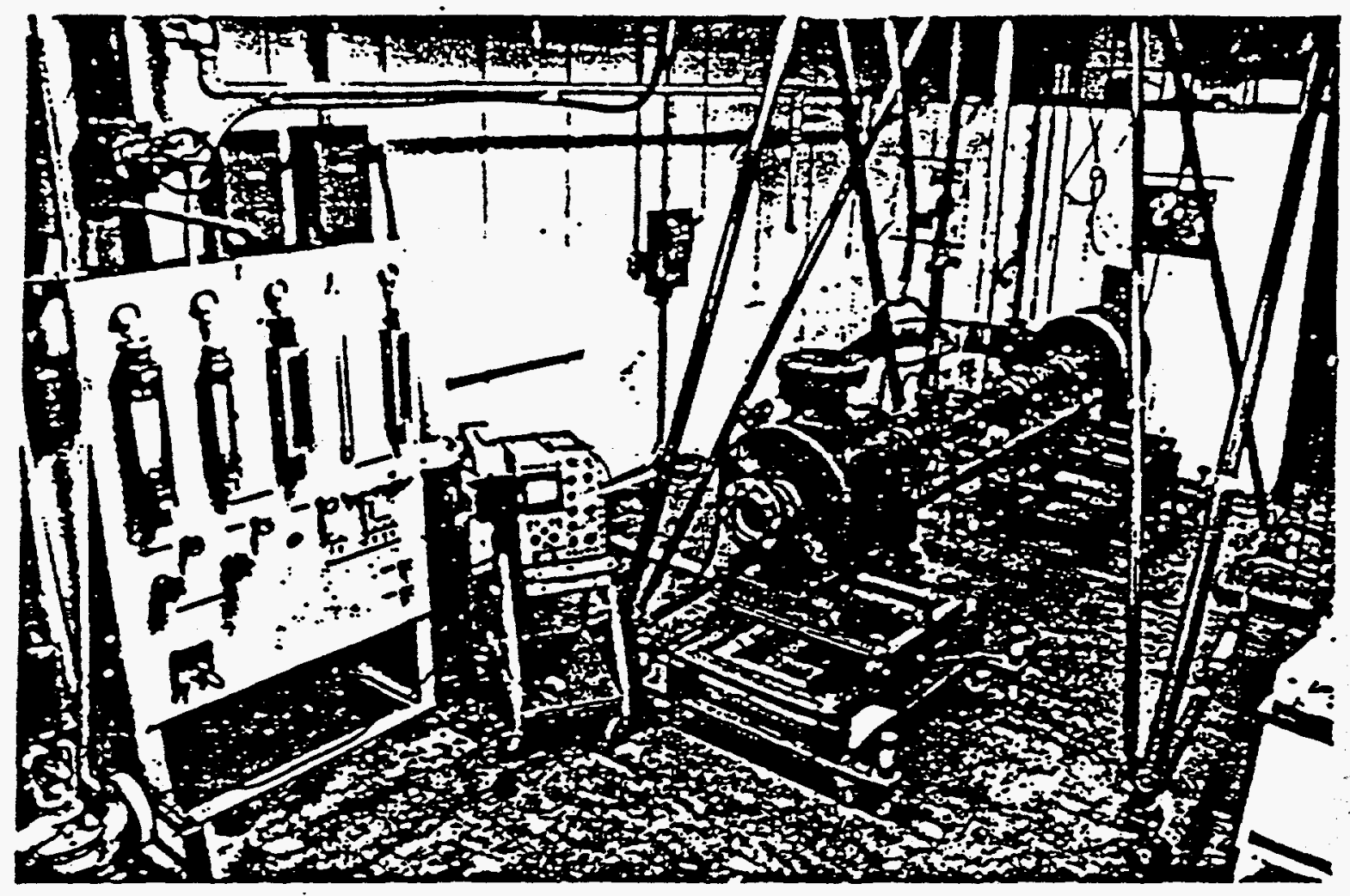

Fig. 9 A Copy of a Photograph of the Developed Experimental Setup, its Two Support Stands and the Control and Measurements Panel. 


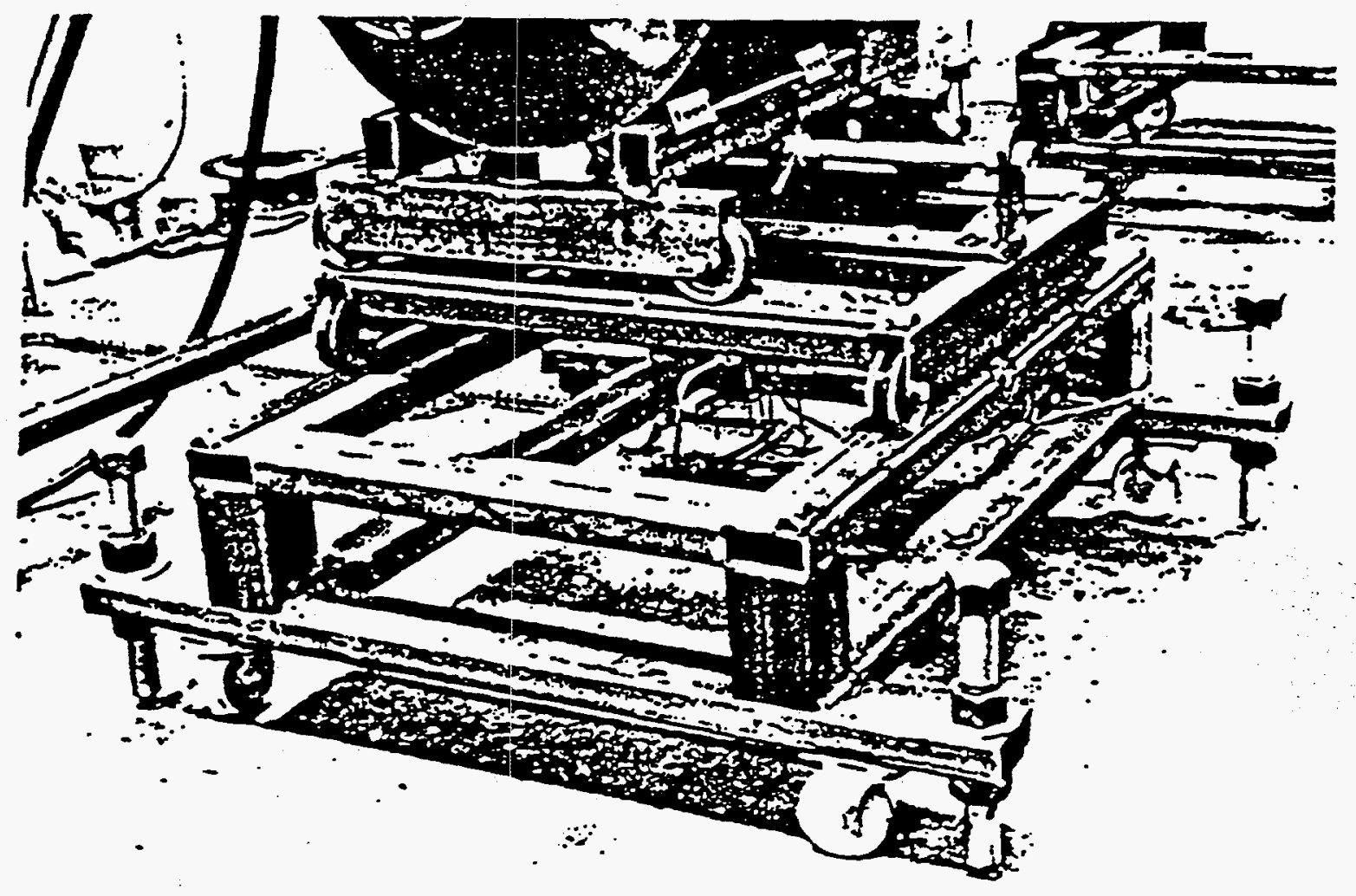

Fig. 10 A Copy of a Photograph of One of the Two Support Stands which Can Move the Pulse Combustor in Three Mutually Perpendicular Directions. 

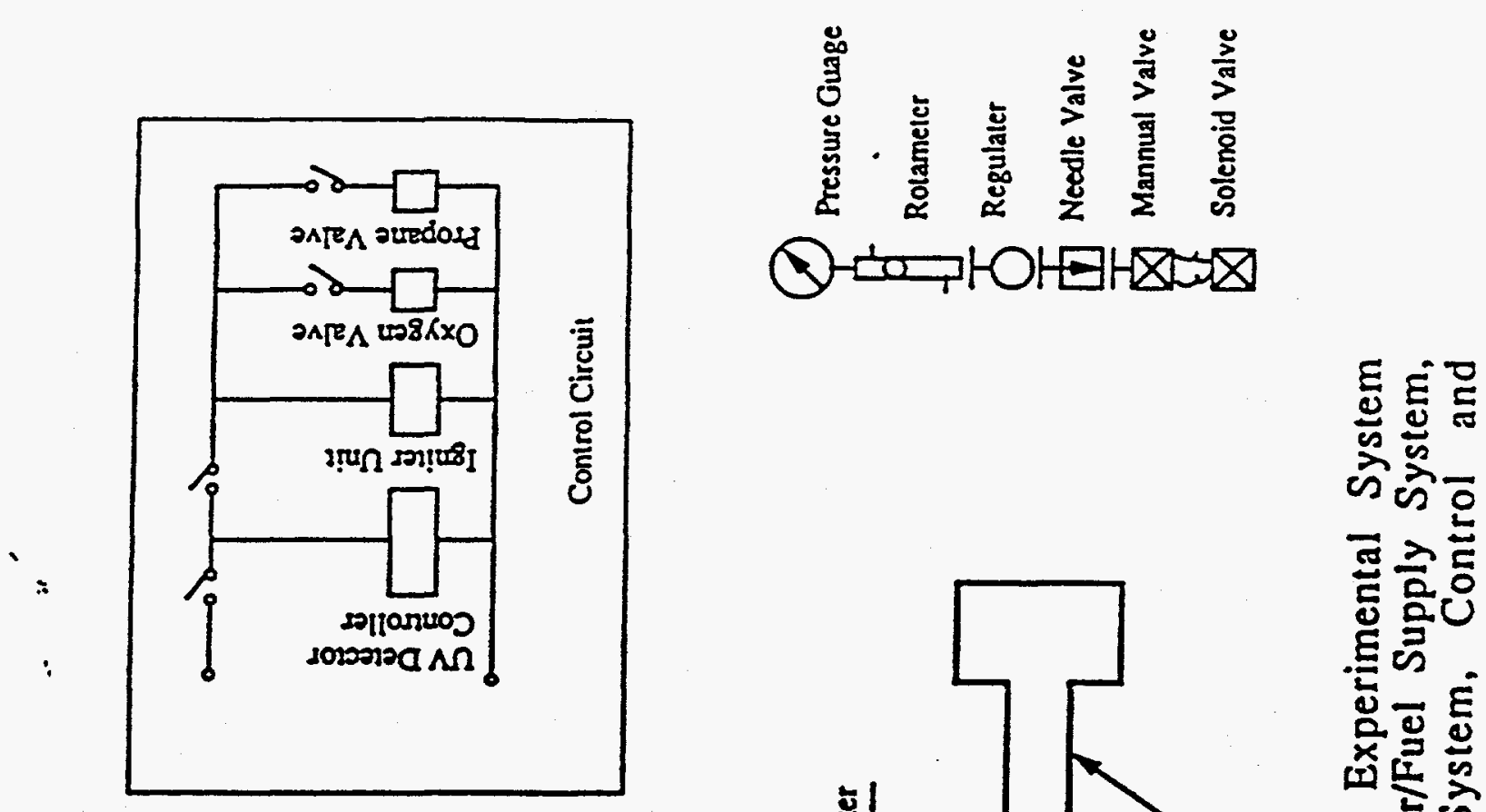

品

के के

폴 $\frac{2}{5}$

응

है

ดัญ

(ป) 这出

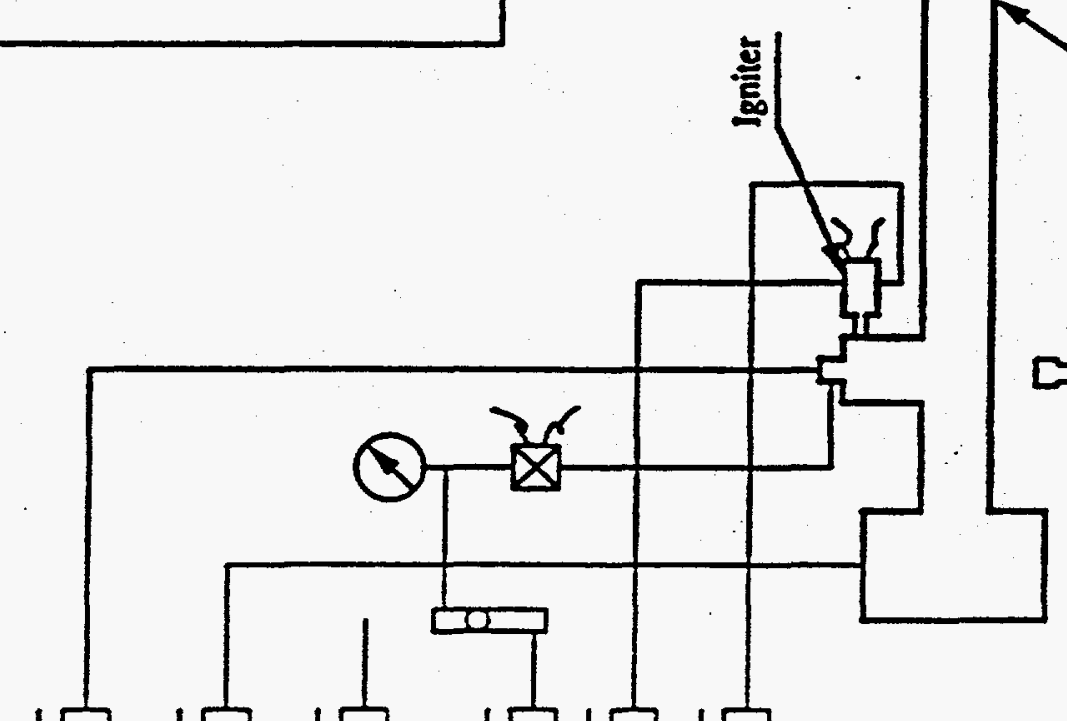

H H H H H

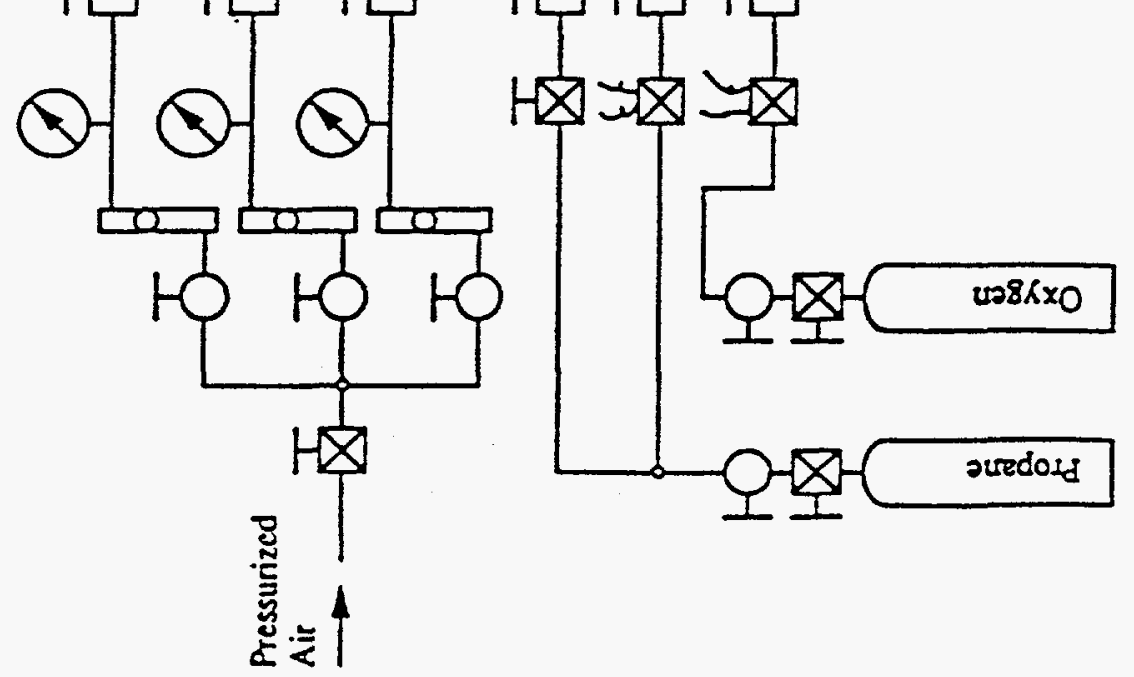

$0 . \frac{5}{2}$

0

눙

雪

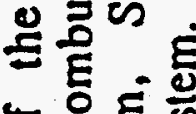

แU⿺ है

u ำ

蛋 部

$I$
$\frac{0}{2}$ 


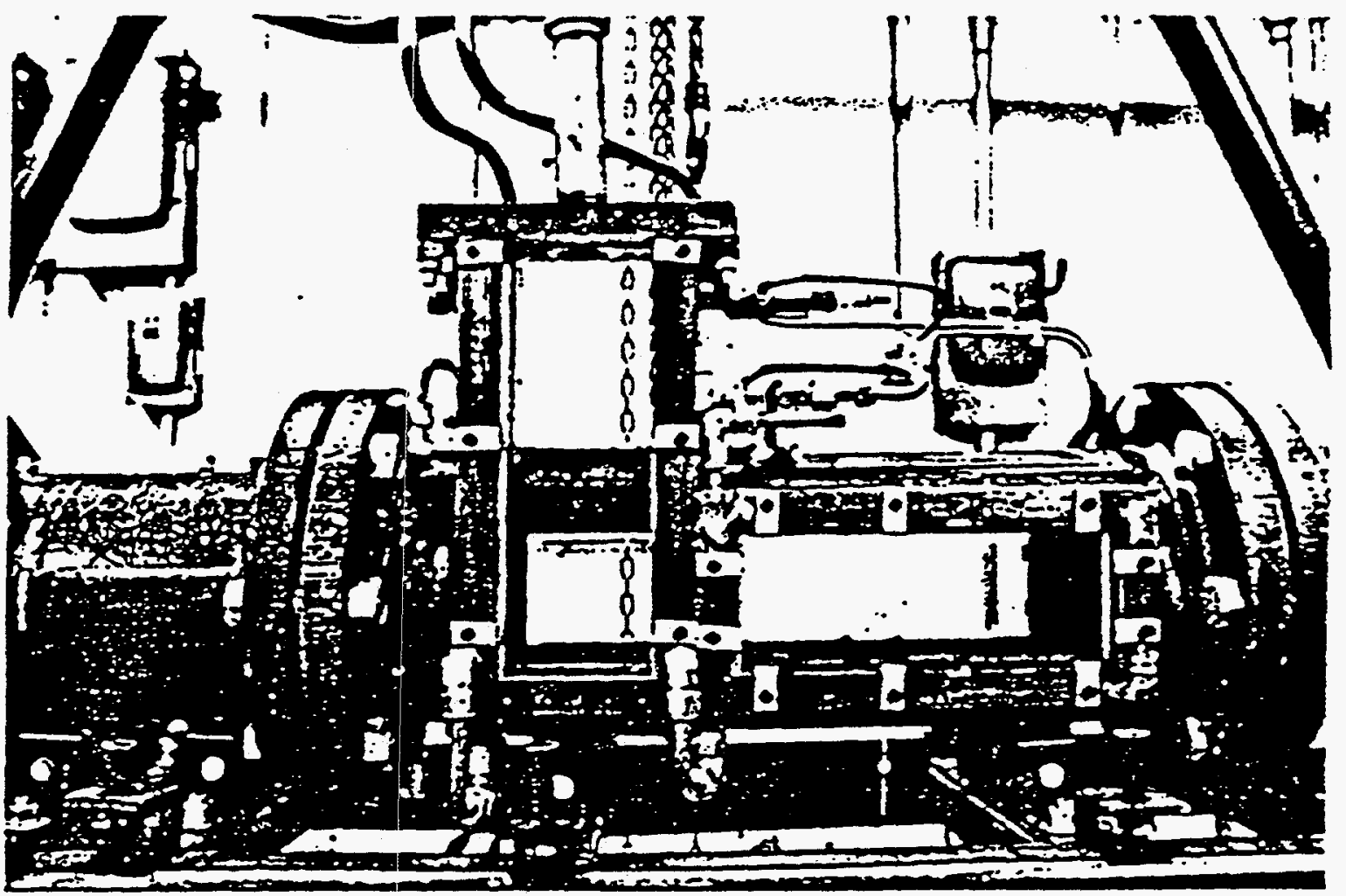

Fig. 12 A Copy of a Photograph of the Air/Fuel Injection Duct and Combustor Sections which Are Water Cooled and Equipped with Quartz Windows. Note the Ignitor on the Right Side of the Injection Duct. 


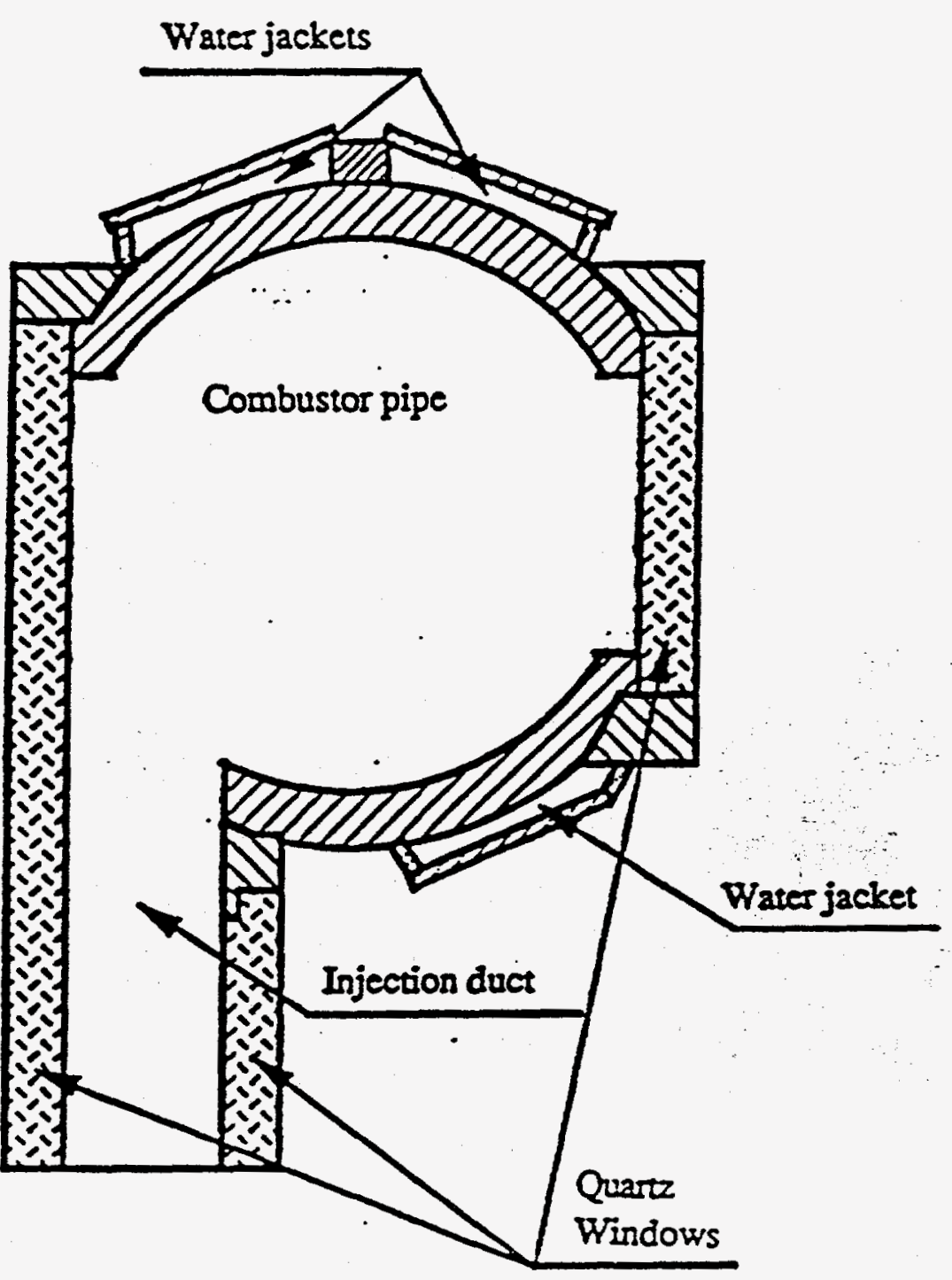

Fig. 13 A Schematic of the Cross Section of the Combustor at the Point Where It Attaches to the Injection System. 


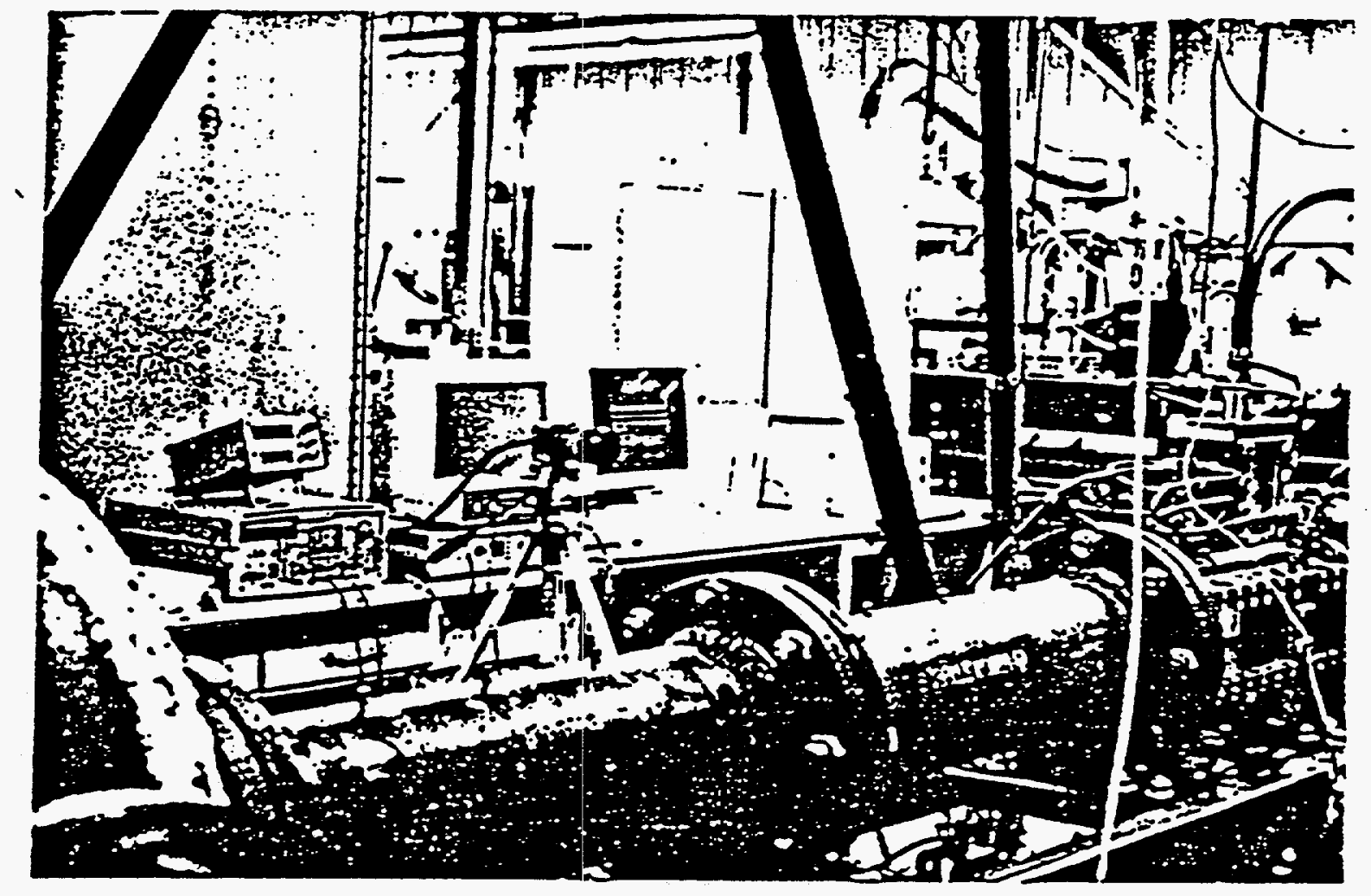

Fig.14 A Copy of a Photograph of the Mini-Computer Station Based Imaging System with the Intensified CCD Camera. 


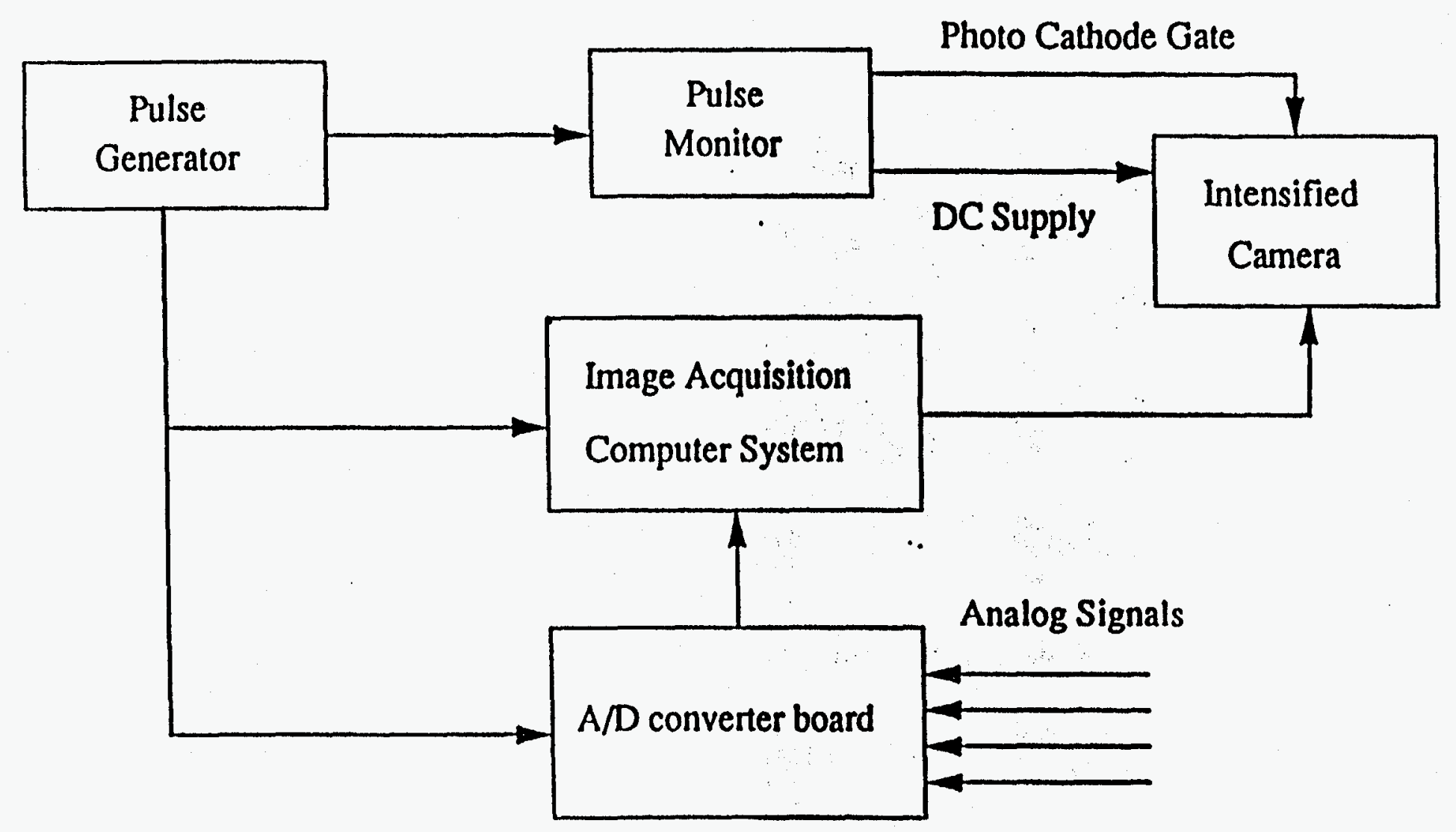

Fig. 15 A Schematic of the Mini-Computor Based Imaging System with the Intensified CCD Camera and Four Analog Signal Input Channels. 

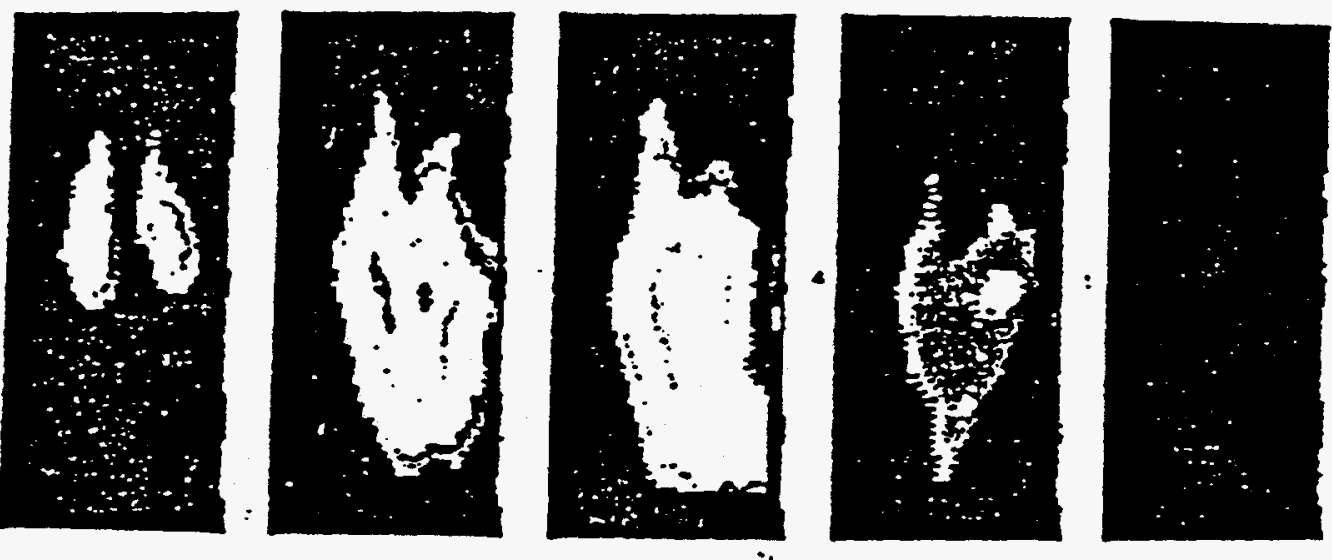

Fig. 16 Images of Visible Radiation Intensity Distributions inside - the Air/Fuel Injection Duct Obtained with the Imaging System in an Experiment Conducted with a Propane Input Rate of 0.94 SCFM and a Nondimensional Air/Fuel Ratio of 1.35. All Five Frames, from the Left to Right, Were Taken in the Same Cycle at Equal Time Intervals. 

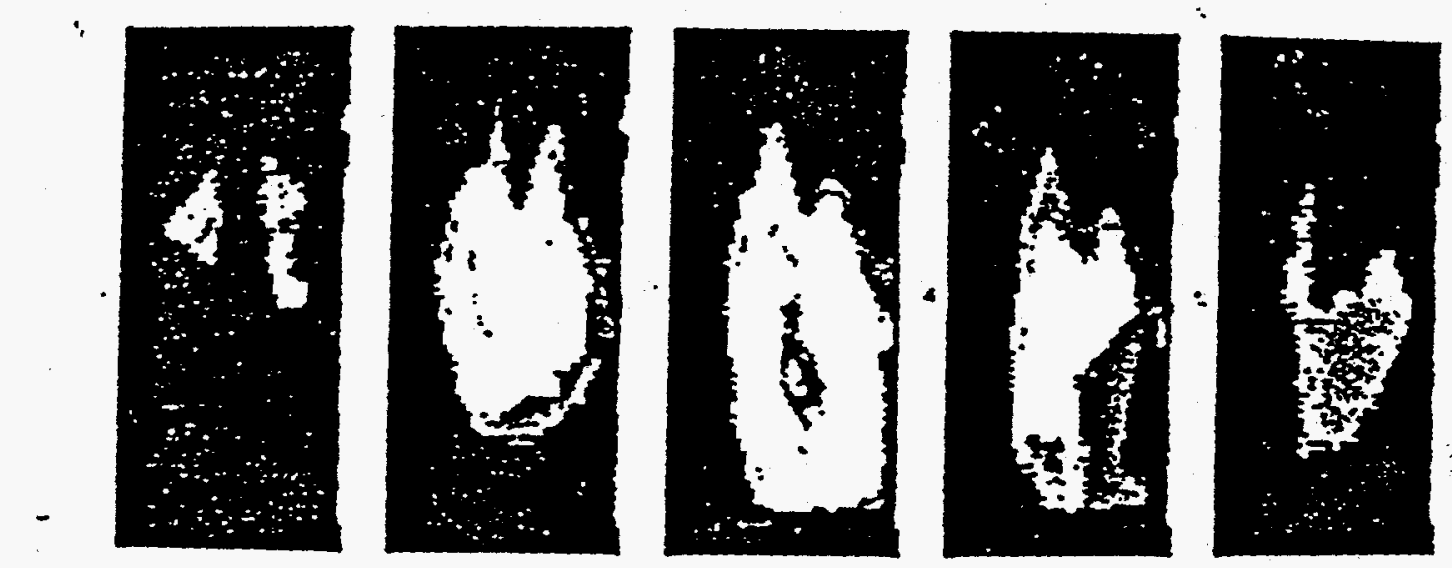

Fig. 17 Images of Visible Radiation Intensity Distributions inside the Air/Fuel Injection Duct Obtained with the Imaging System in an Experiment Conducted with a Propane Input Rate of 0.94 SCFM and a Nondimensional Air/Fuel Ratio of 1.35. All Five Frames, from the Left to Right, Were Taken in the Same Cycle at Equal Time Intervals. 

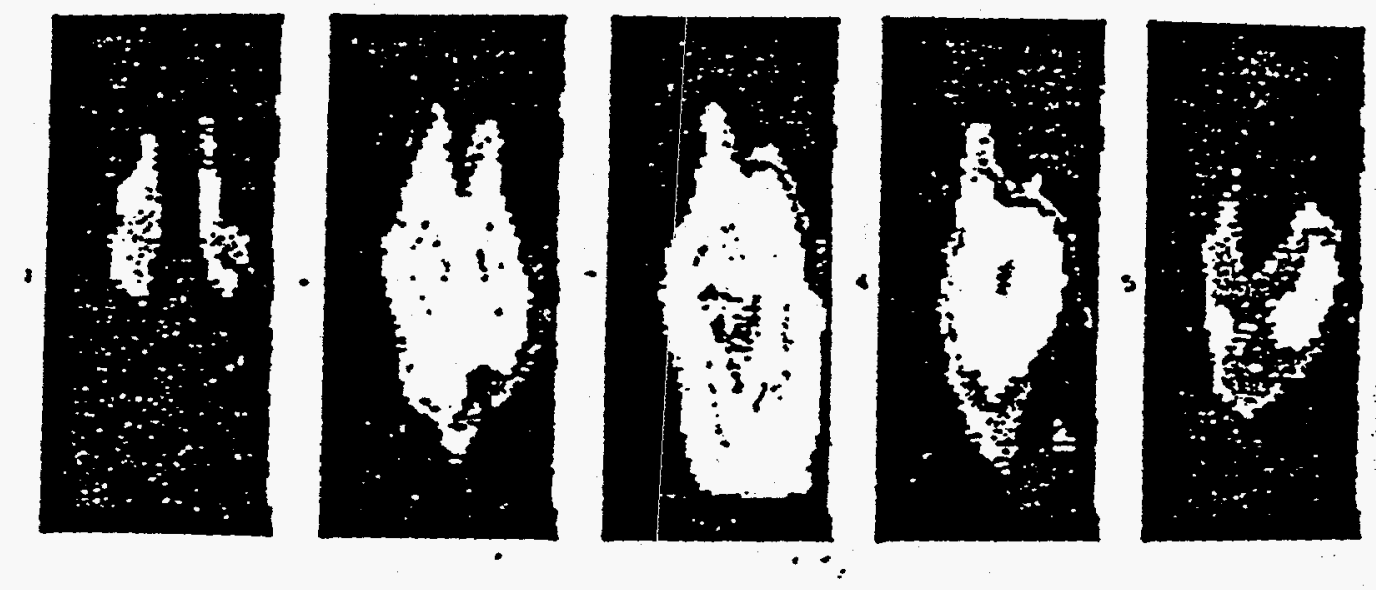

Fig. 18 Images of Visible Radiation Intensity Distributions inside the Air/Fuel Injection Duct Obtained with the Imaging System in an Experiment Conducted with a Propane Input Rate of 0.94 SCFM and a Nondimensional Air/Fuel Ratio of 1.35. These Five Frames, from the Left to Right, Were Taken at Equal Time Intervals in a Pressure Cycle next to the One Shown in Fig. 17. 

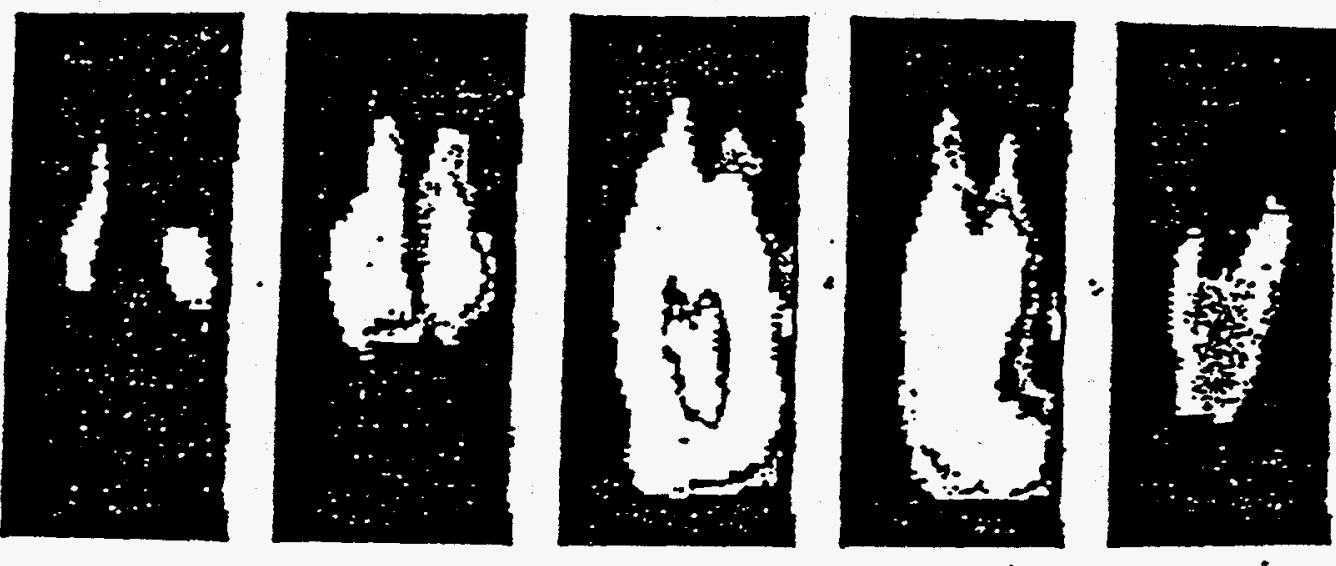

Fig. 19 Images of Visible Radiation Intensity Distributions inside the Air/Fuel Injection Duct Obtained with the Imaging System in an Experiment Conducted with a Propane Input Rate of 0.94 SCFM and a Nondimensional Air/Fuel Ratio of 1.35. These Five Frames, from the Left to Right, Were Taken at Equal Time Intervals in a Pressure Cycle next to the One Shown in Fig. 18. 

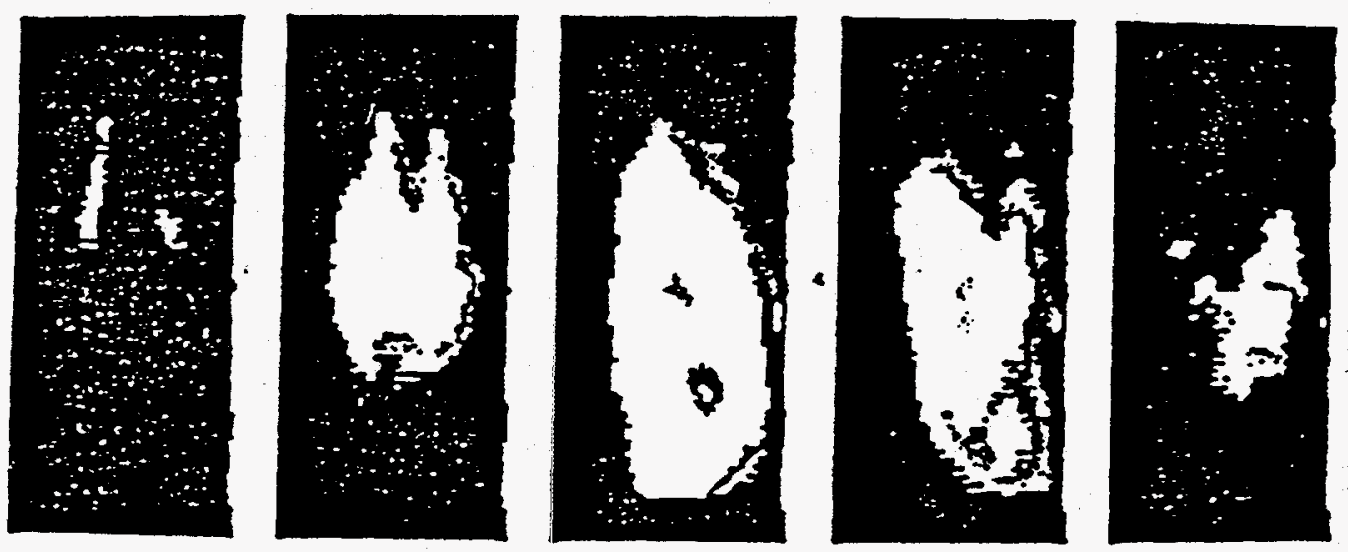

Fig. 20 . Images of Visible Radiation Intensity Distributions inside the Air/Fuel Injection Duct Obtained with the Imaging System in an Experiment Conducted with a Propane Input Rate of 0.94 SCFM and a Nondimensional Air/Fuel Ratio of 1.35. These Five Frames, from the Left to Right, Were Taken at Equal Time Intervals in a Pressure Cycle next to the One Shown in Fig. 19. 

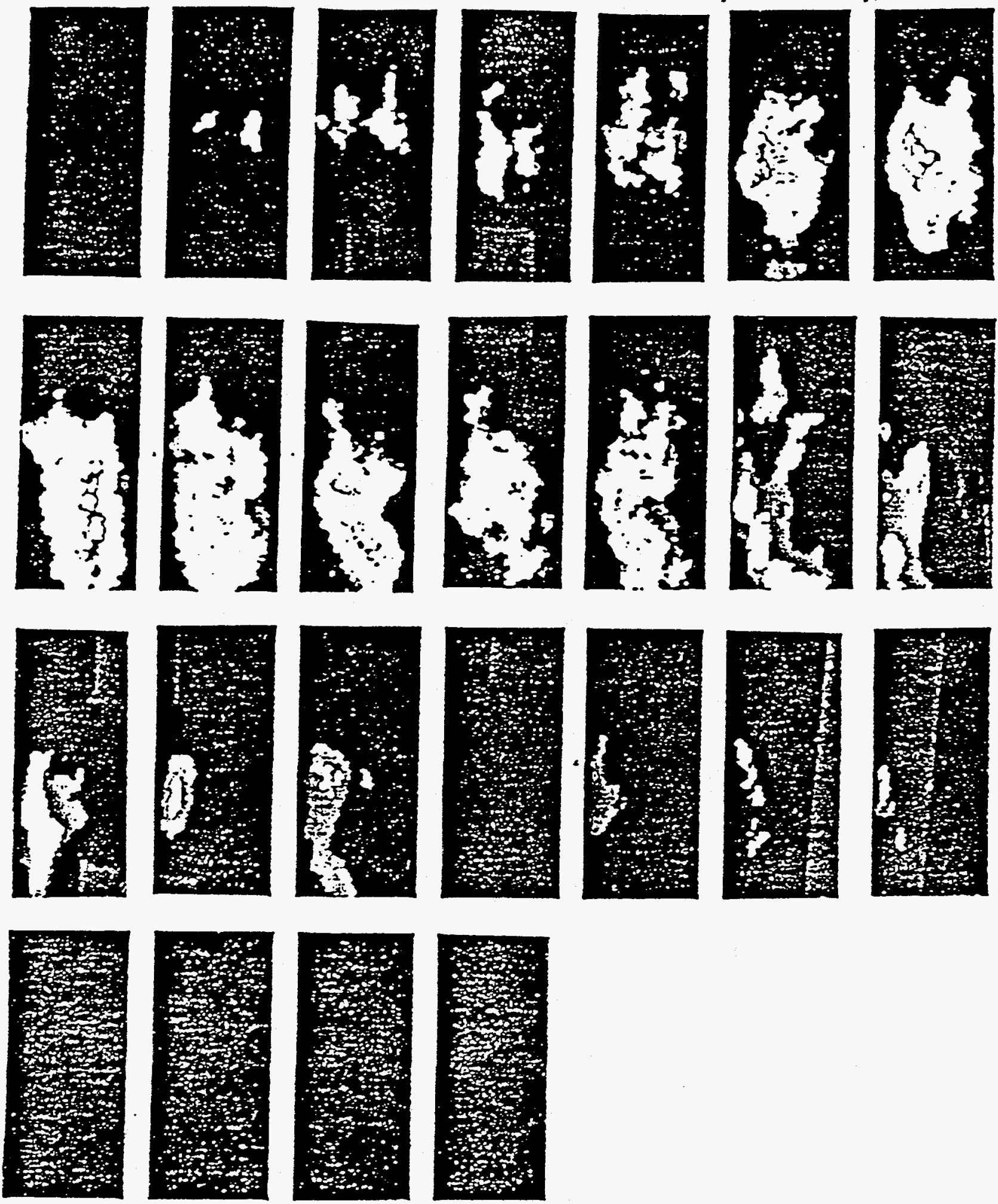

Fig. 21 Images of Visible Radiation Intensity Distributions inside the Air-Fuel Injection Duct Obtained with the Imaging System. Operated on the Stroboscopic Mode in an Experiment Conducted with a Propane Input Rate of 0.94 SCFM and Nondimensional Air/Fuel Ratio of 1.35 . Note that It provides 25 Frames for One Cycle. 

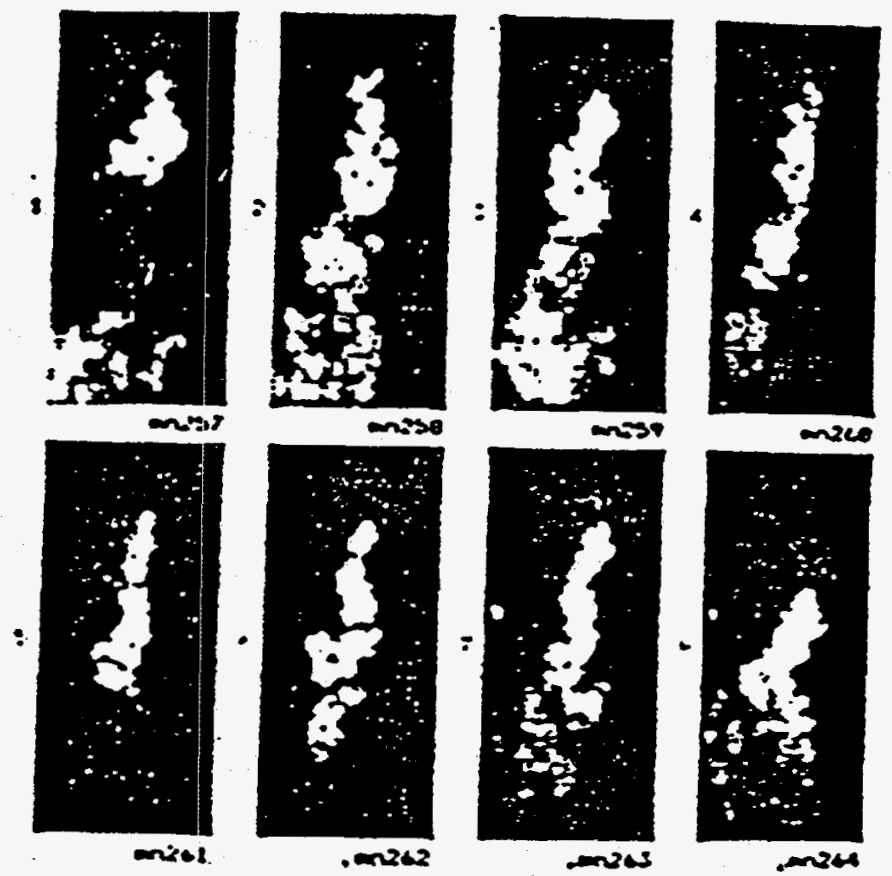

Fig. 22 Images of Visible Radiation Intensity Distributions inside the Air/Fuel Injection Duct Obtained with the Imaging System in an Experiment Conducted under NonPulsating Operation. The Time Interval between Two Frames Is $2 \mathrm{~ms}$. 

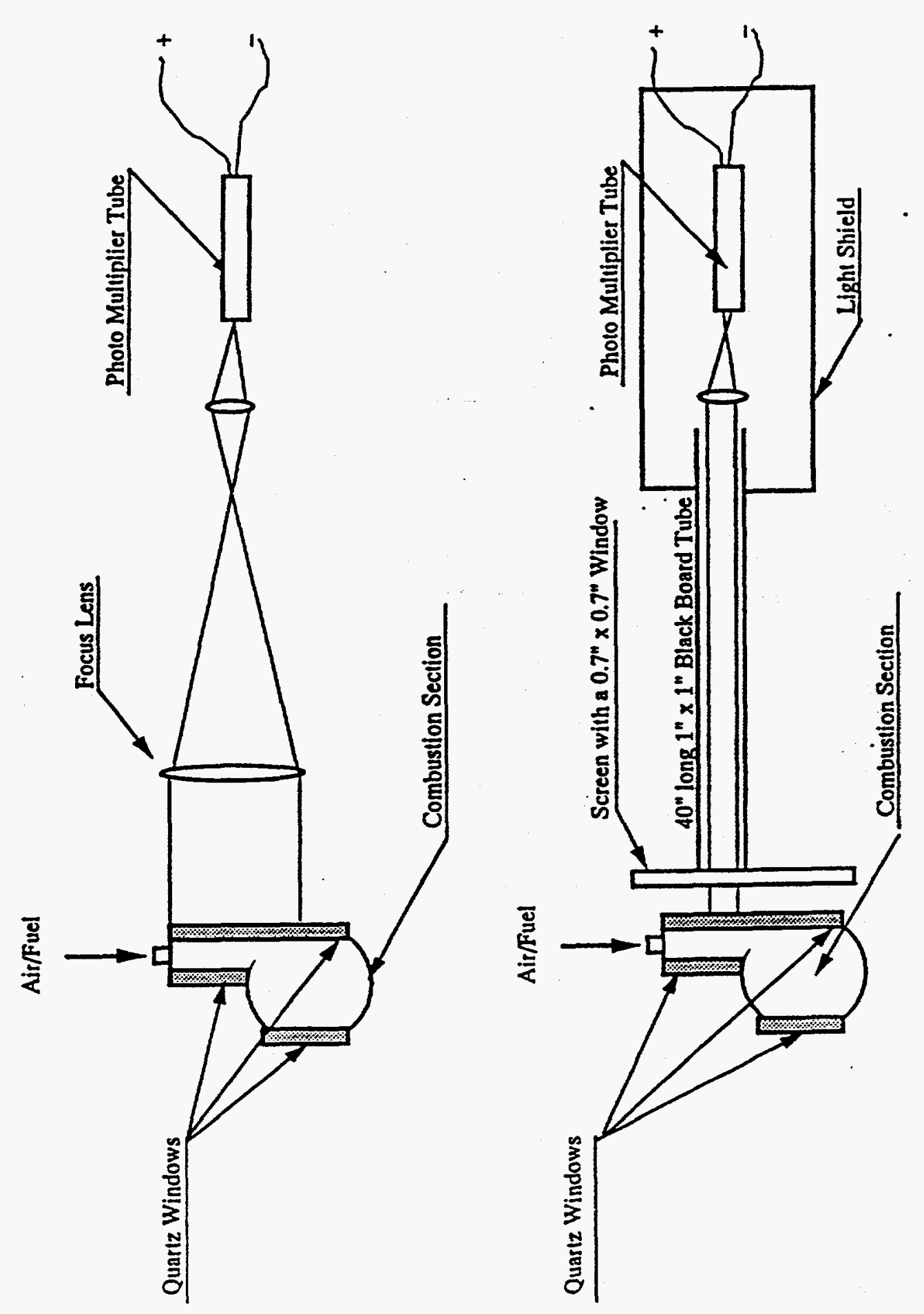

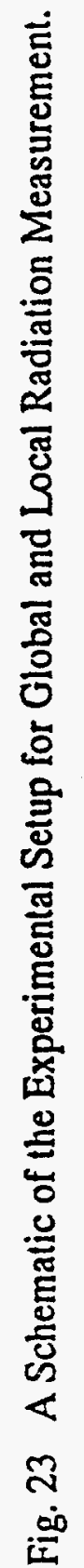




\section{Fuel/Air Mixture}

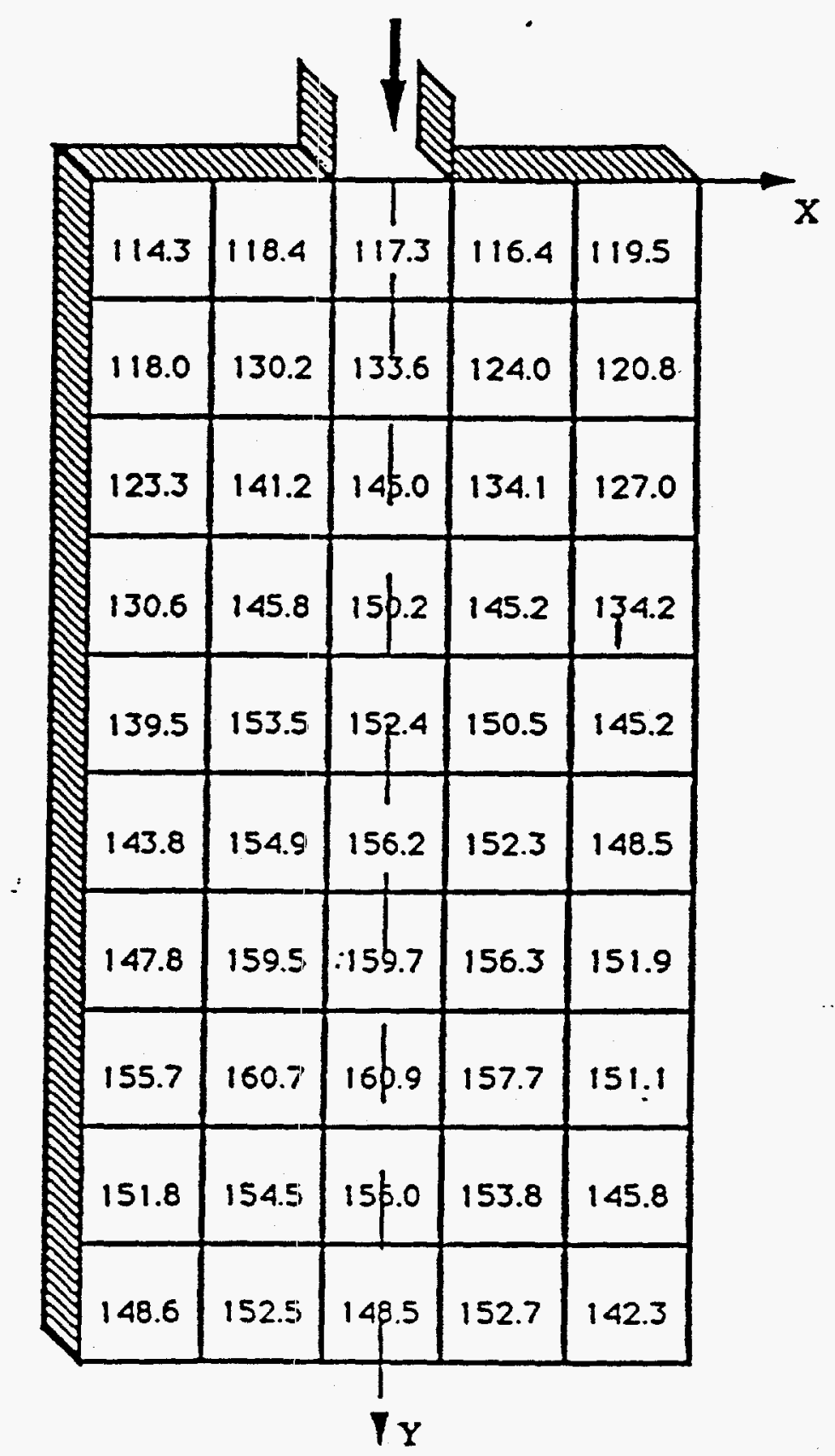

Fig. 24 Distribution of the Local C-H Radiation Intensity inside the Air/Fuel Injection Duct Measured in Experiments Conducted with a Propane Input Rate of 1.6 SCFM and a Nondimensional Air/Fuel Ratio of 0.97 . 
Fuel/Air Mixnure

\begin{tabular}{|c|c|c|c|c|}
\hline-10.0 & -5.8 & -24.7 & -17.8 & -8.5 \\
\hline-6.2 & -45.1 & -56.3 & $-5.2^{\circ}$ & -2.2 \\
\hline-22.8 & -65.1 & $-5 p .5$ & -35.0 & -23.9 \\
\hline-39.8 & -72.9 & & -33.4 & -69.2 \\
\hline-51.8 & -51.3 & -11.8 & -33.2 & -70.2 \\
\hline-41.5 & -40.1 & -0.5 & -24.0 & -60.6 \\
\hline-42.0 & -25.3 & $+2 b .9$ & -17.1 & -48.4 \\
\hline-37.1 & -7.3 & $:+2 \beta .5$ & +0.1 & -34.9 \\
\hline-45.5 & -2.6 & $+5 \$ .1$ & +15.9 & -13.6 \\
\hline-29.5 & -22.8 & +60.3 & +40.0 & +37.9 \\
\hline
\end{tabular}

Fig. 25 Distribution of the Phase Difference between the Local C-H Radiation and Pressure Oscillations inside the Air/Fuel Injection Duct Measured in Experiments Conducted with a Propane Input Rate of 1.6 SCFM and a Nondimensional Air/Fuel Ratio of 0.97 . 


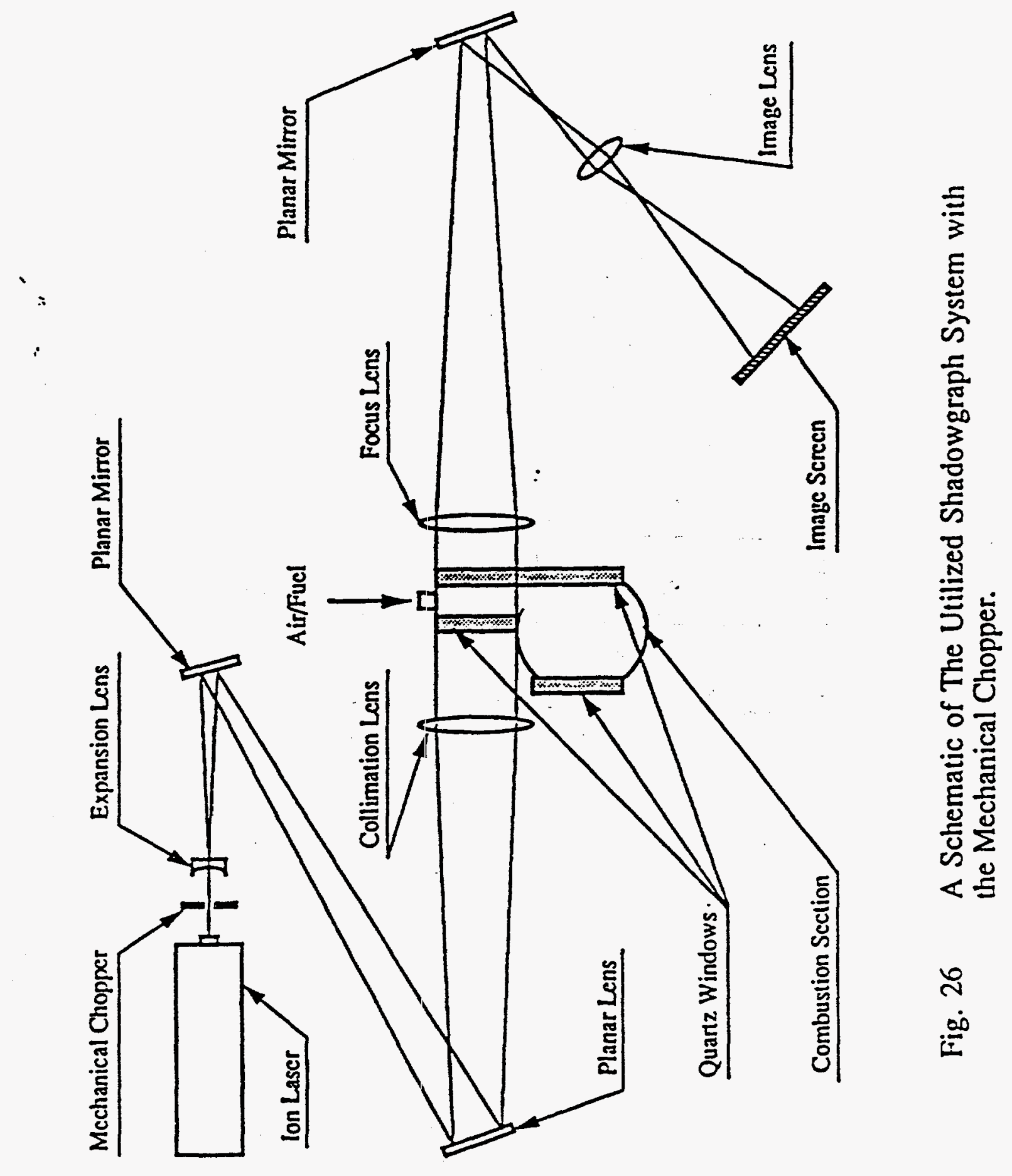




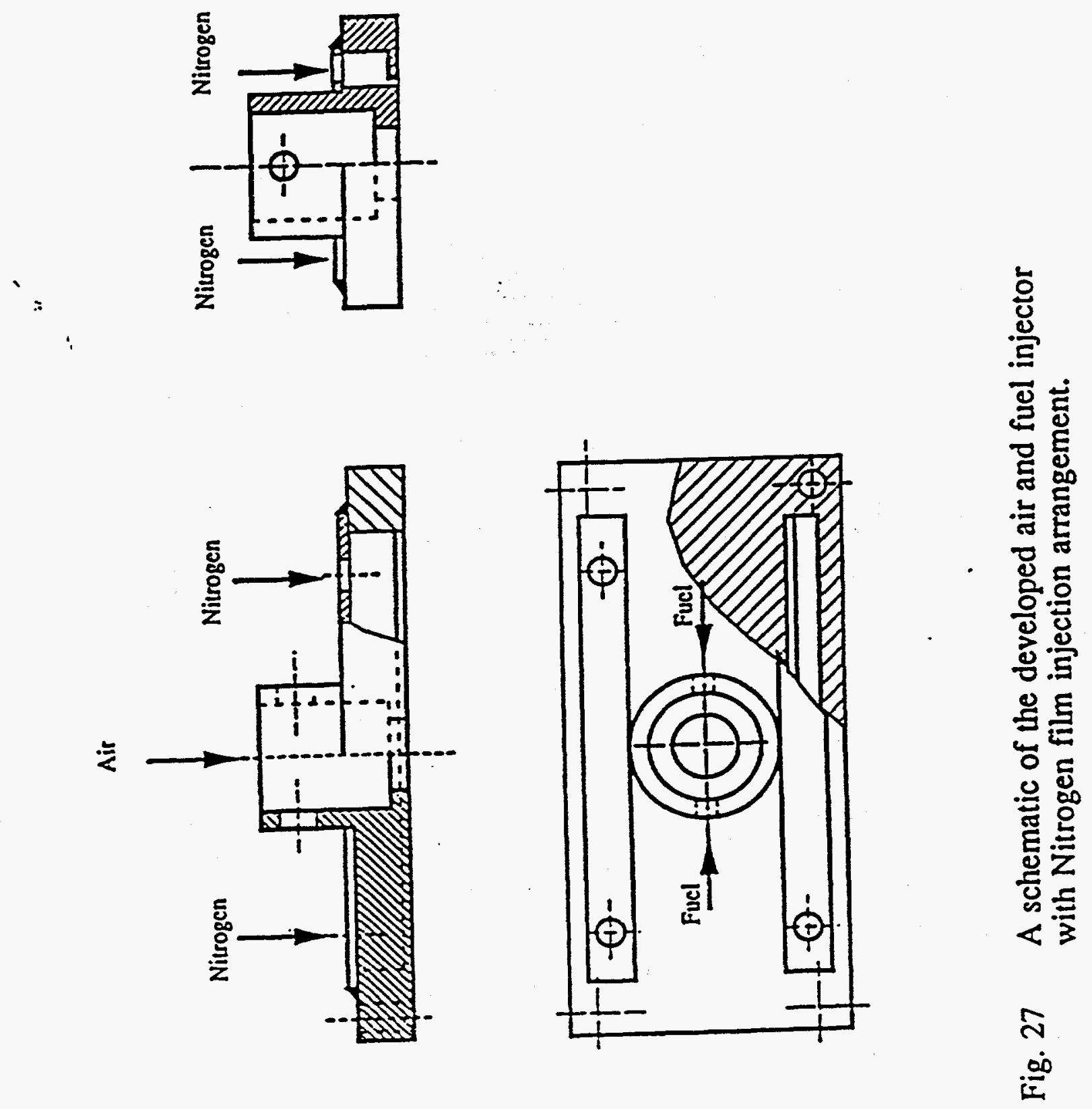




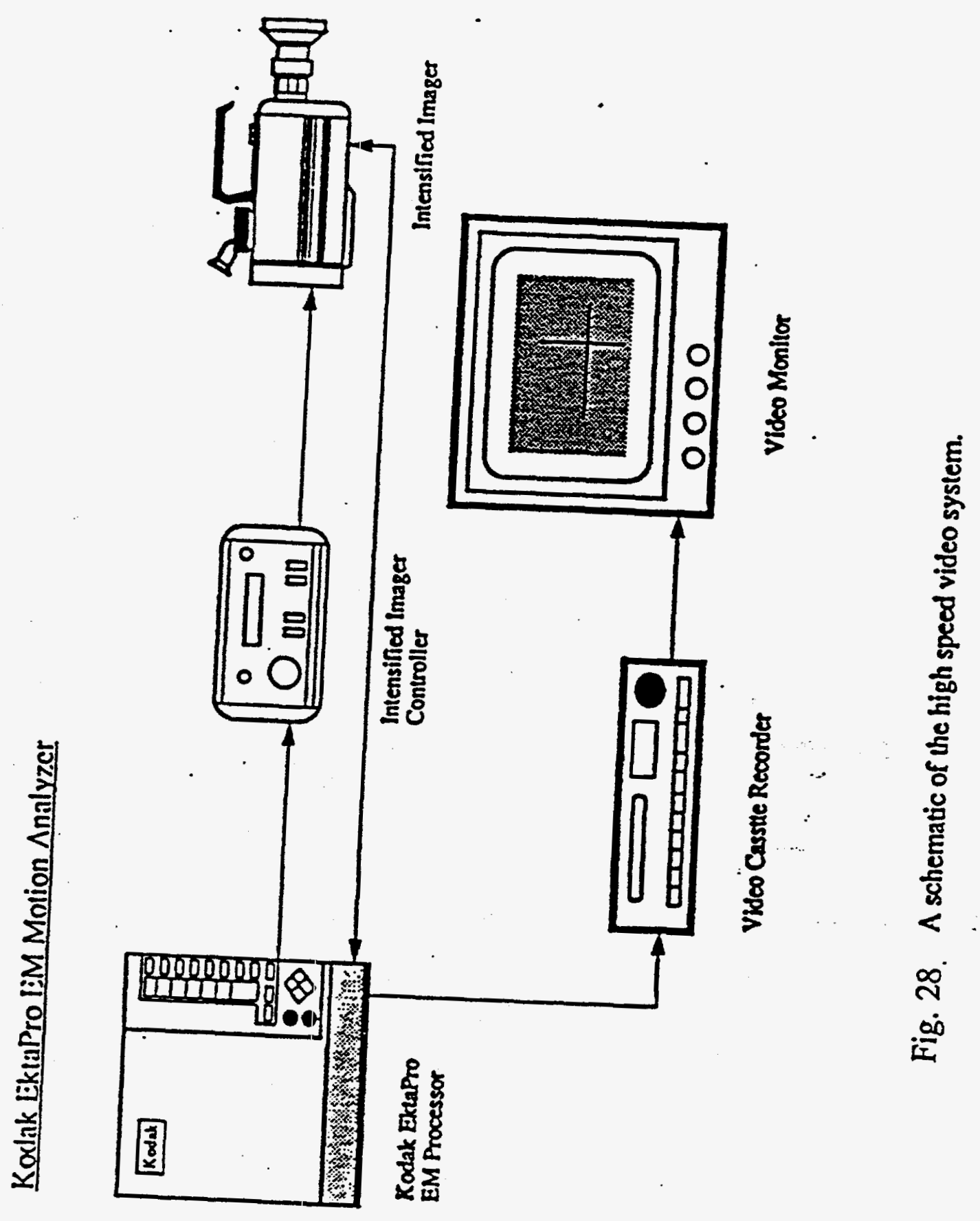




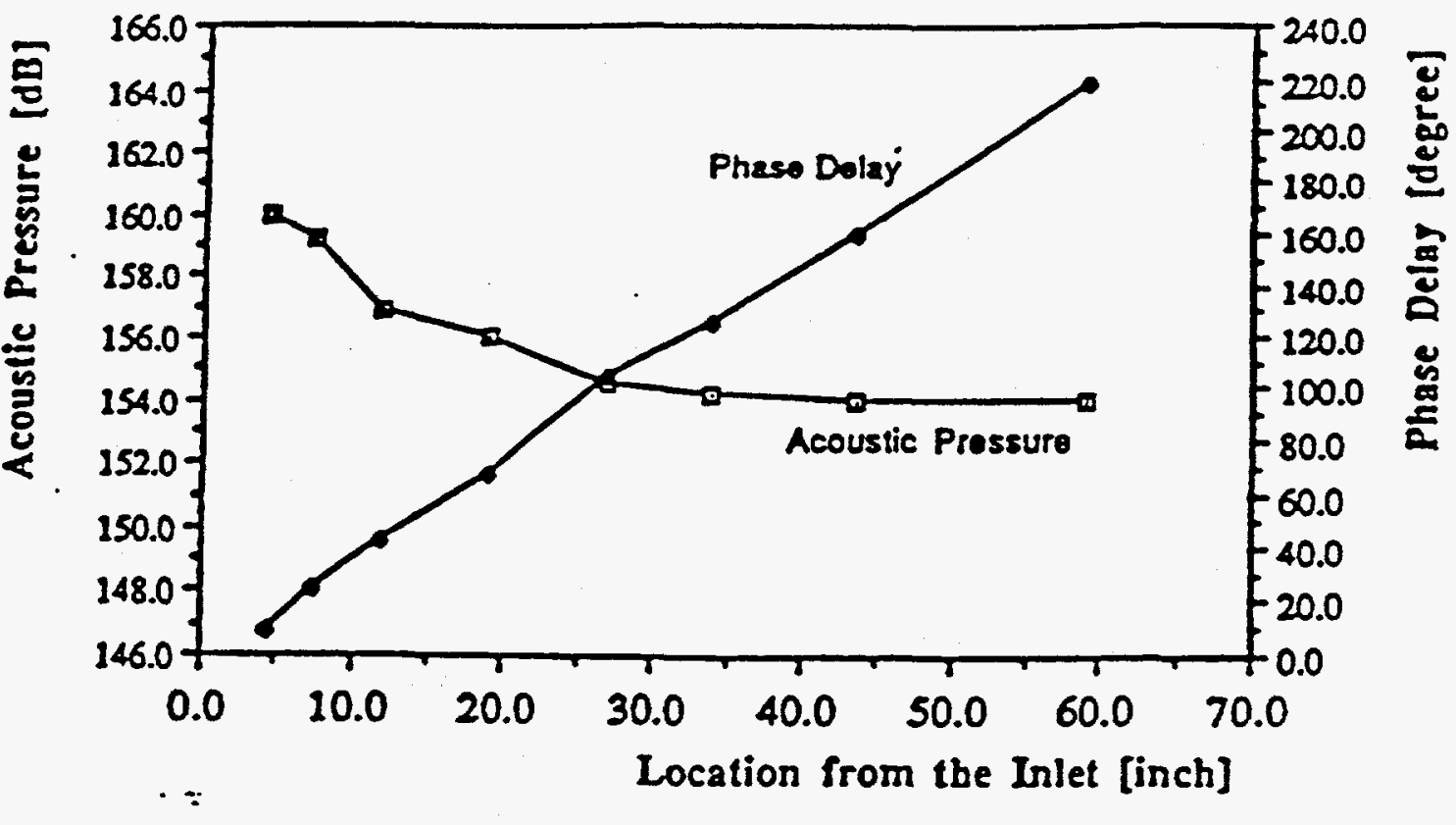

Fig. 29 Dependence of the Amplitude of Acoustic Pressure (in dB) and the Phase Delay upon the Locations along the Air Feed Line Measured in Tests when the Combustor Was Operating on Fundamental Mode of $104 \mathrm{~Hz}$.

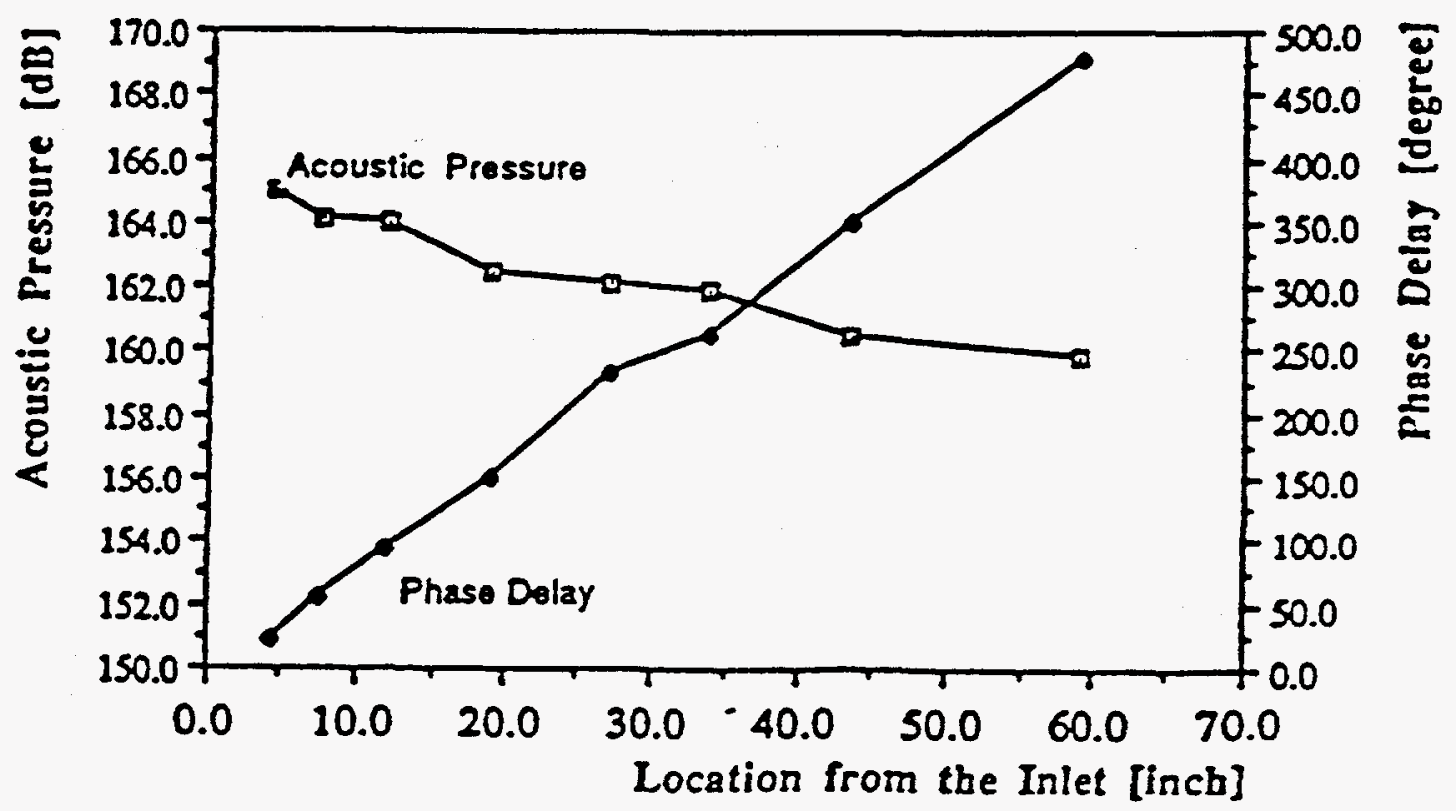

Fig. 30 Dependence of the Amplitude of Acoustic Pressure (in dB) and the Phase Delay upon the Locations along the Air Feed Line Measured in Tests when the Combustor Was Operating on First Harmonic Mode of $230 \mathrm{~Hz}$. 


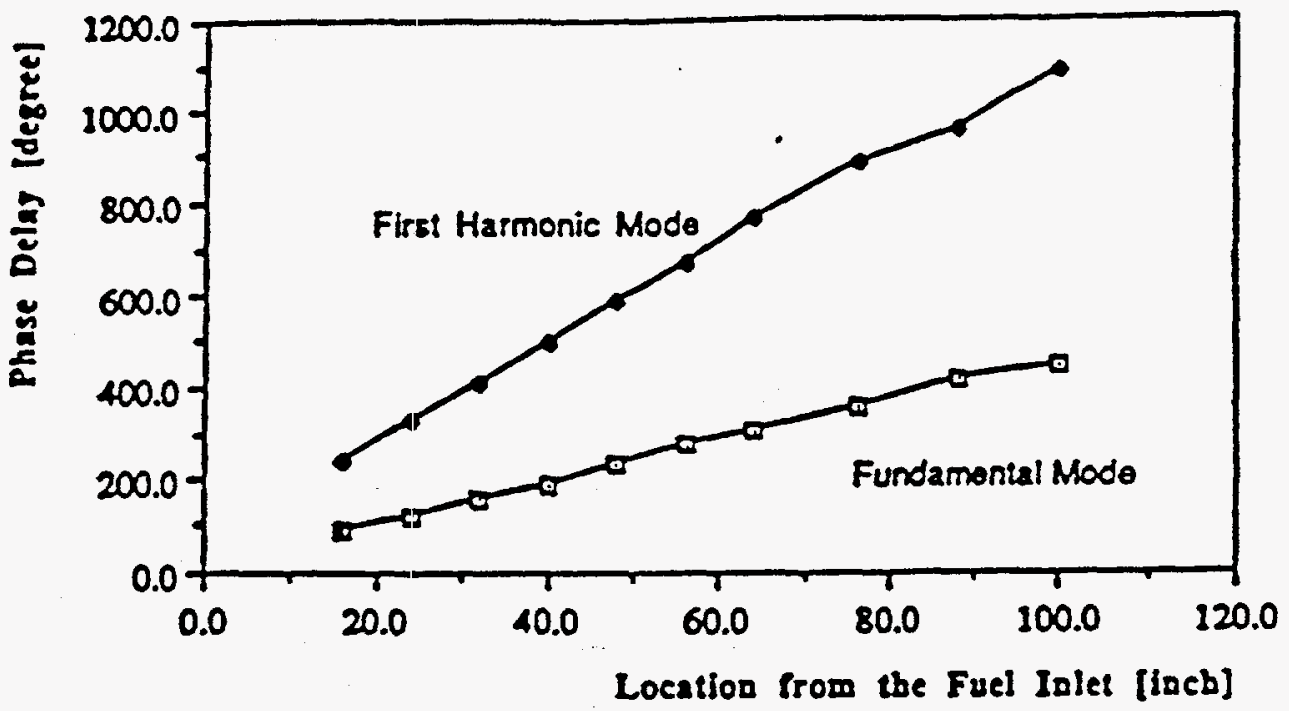

Fig. 31 Dependence of the Phase Delay upon the Locations along the Fuel Feed Line Measured in Tests when the Combustor Was Operating on Fundamental and First Harmonic Modes, respectively.

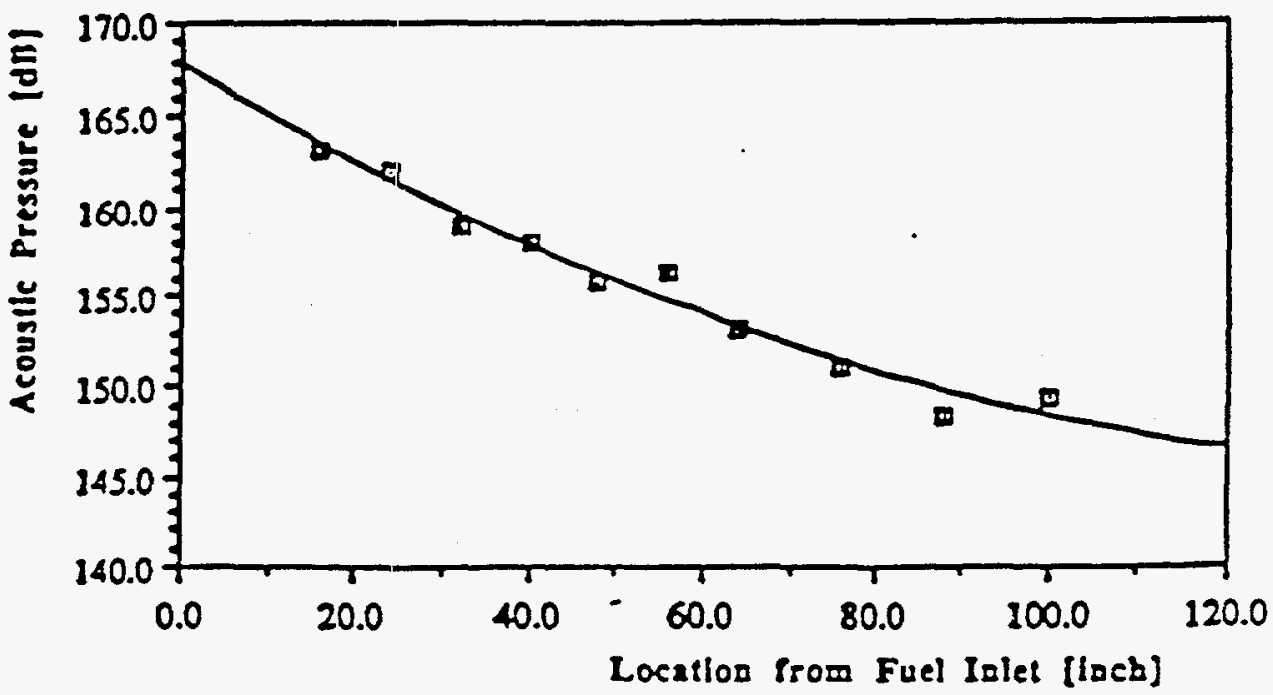

Fig. 32 Dependence of the Amplitude of Acoustic Pressure (in dB) upon the Locations along the Fuel Feed Line Measured in Tests when the Combustor Was Operating on First Harmonic Mode of $230 \mathrm{~Hz}$. 


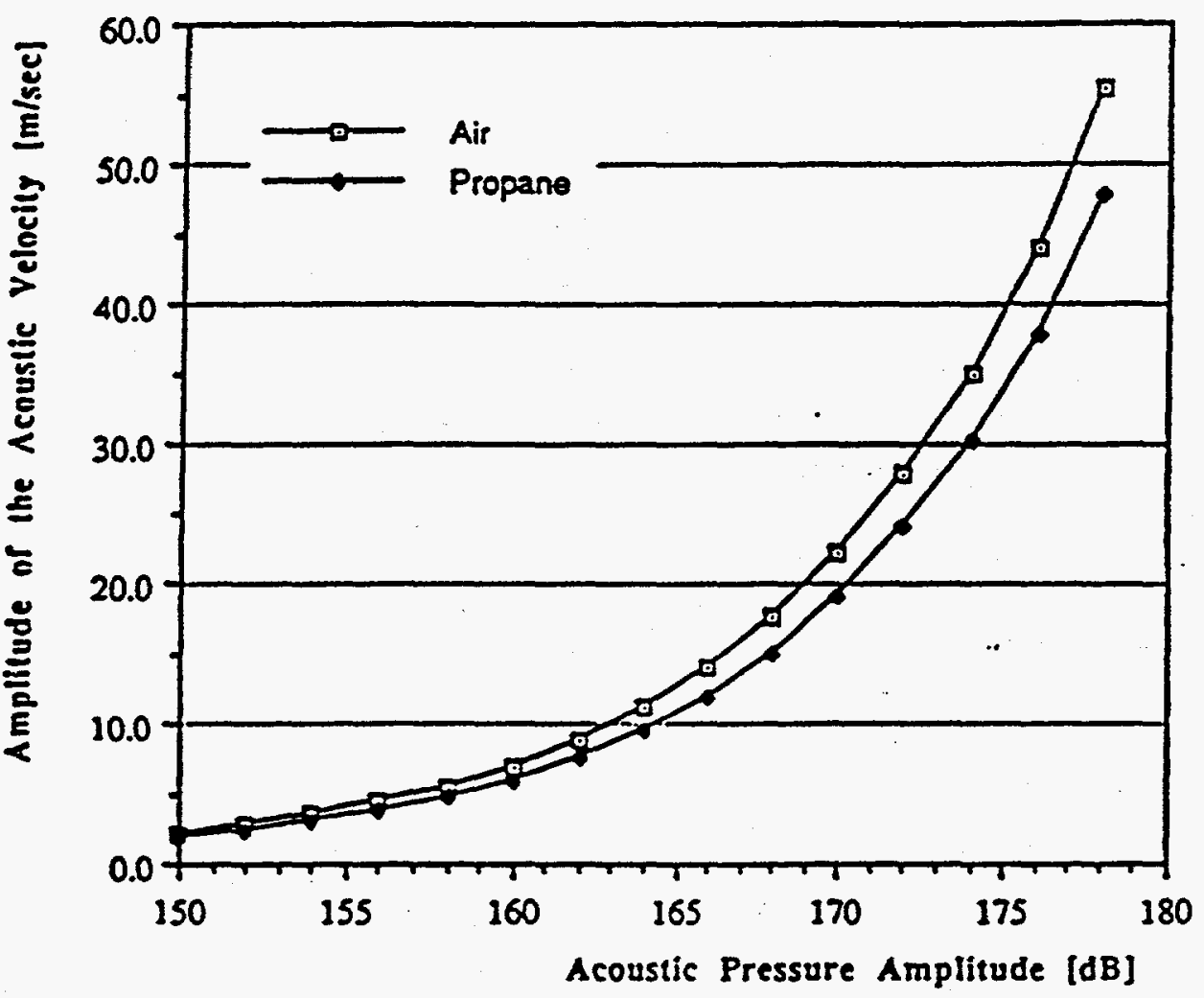

Fig. 33 Dependence of the Amplitude of the Acoustic Velocity upon the Amplitude of Acoustic Pressure Oscillarion Calculated for Air and Propane. 


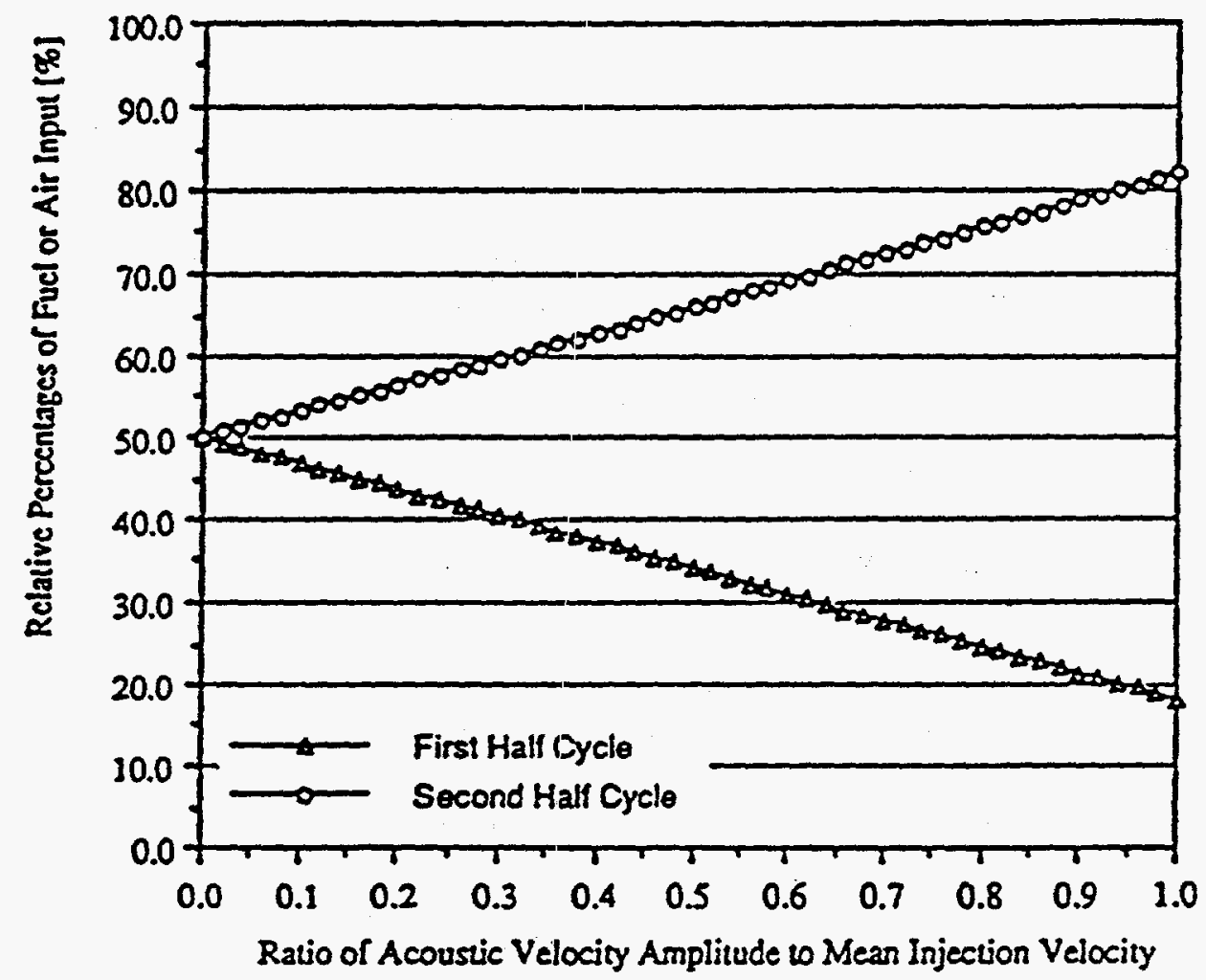

Fig.34 Dependence of the Relative Percentages of Fuel or Air Inputs in the First and Second Half Pressure Cycles upon the Ratio of Acoustic Velocity Amplitude to the Mean Injection Velocity. 


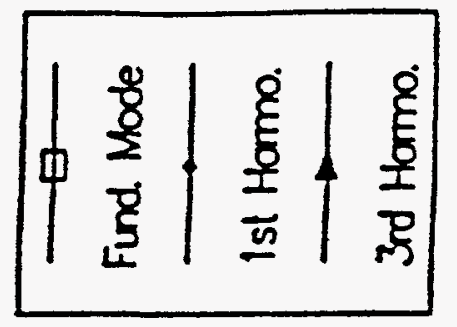

혼ำ

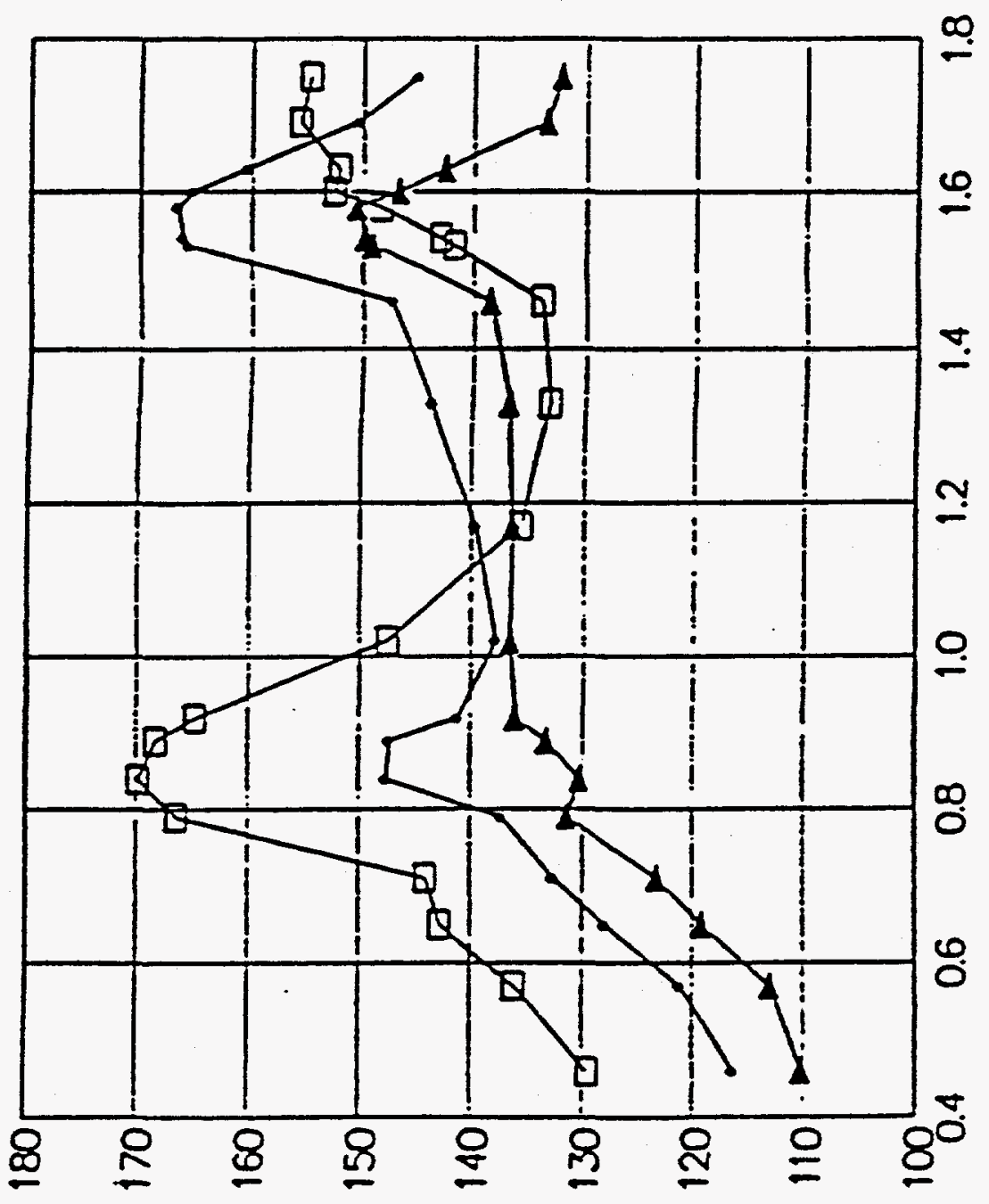

음

드응

«

$8 \approx$

플

을

ธิ้ว 뽐

造

$\frac{5}{1}$
$\frac{2}{2}$
$\frac{\alpha}{\alpha}$
$\frac{\alpha}{2}$
$\frac{\alpha}{2}$

항

题

章

도

ํํํ

흘 营

호용

이응

을월

를 亮

$<\frac{1}{\sqrt{2}}$

은 을

을

论

몽응

पू

응를

m

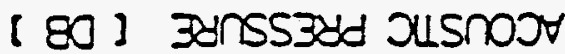




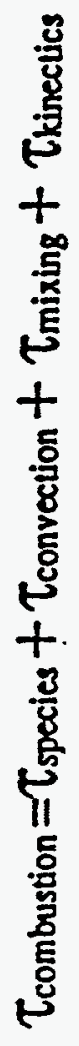

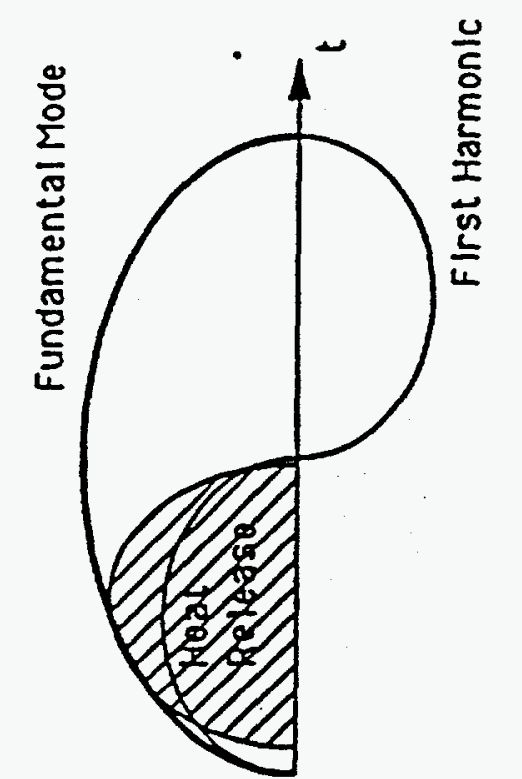

$\frac{0}{8}$

$\pm$

s

릉

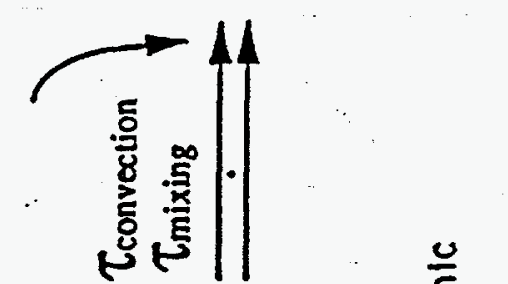

옹

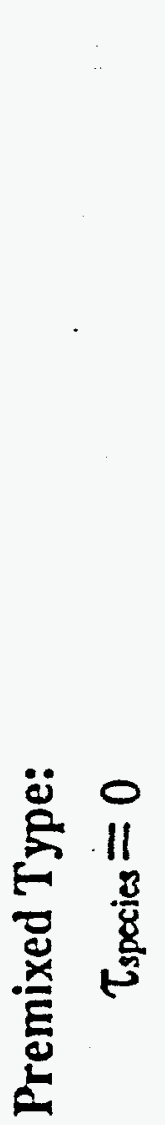

롤

눙

을

둘

E.

을

$<\frac{5}{\infty}$

$m$

is 


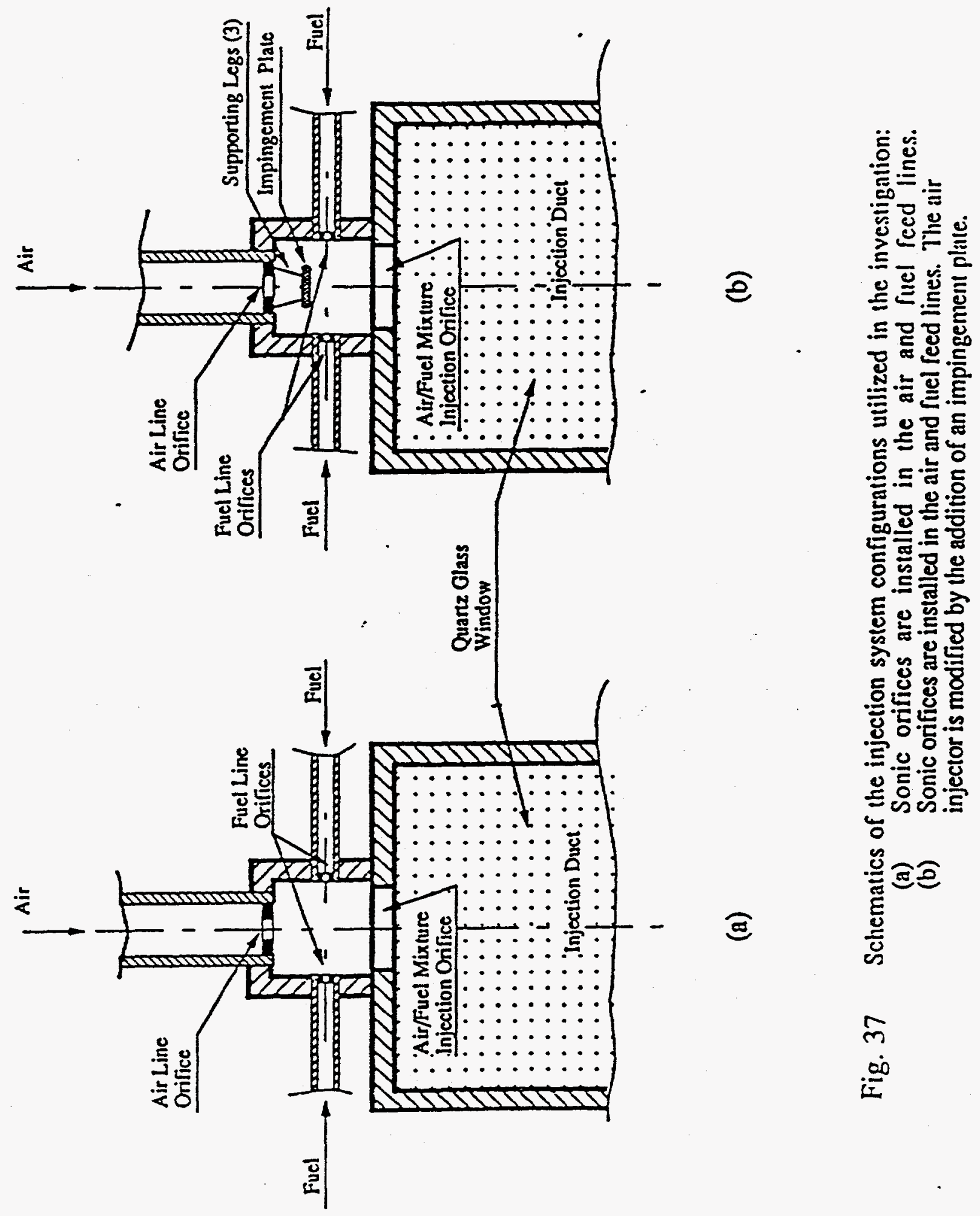




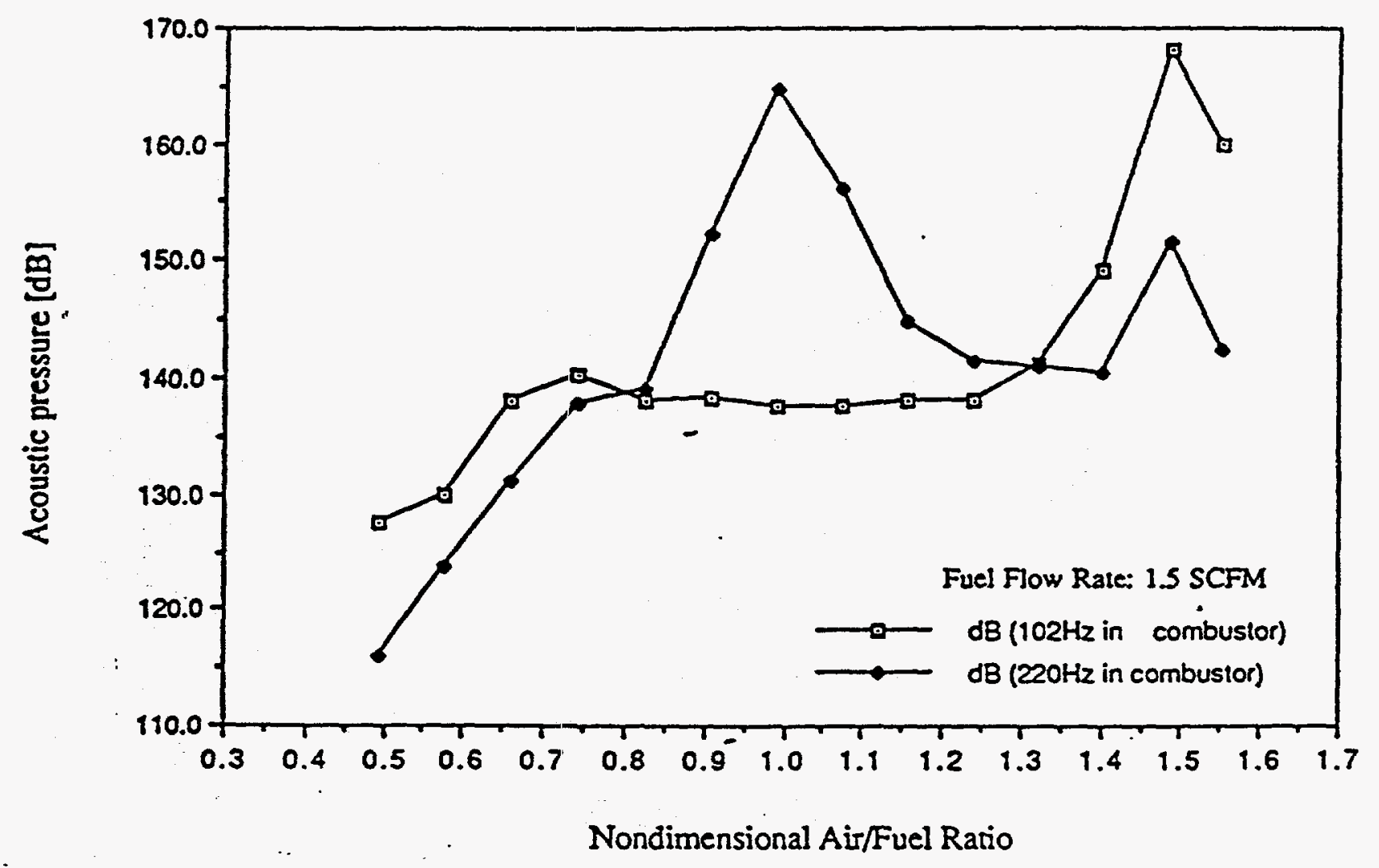

Fig. 38 Dependence of the amplitude of pulsations of the combustor fundamental and first harmonic mode oscillations upon the nondimensional air/fuel ratio measured in tests with 1.5 SCFM propane input rate and the regular injection configuration. 


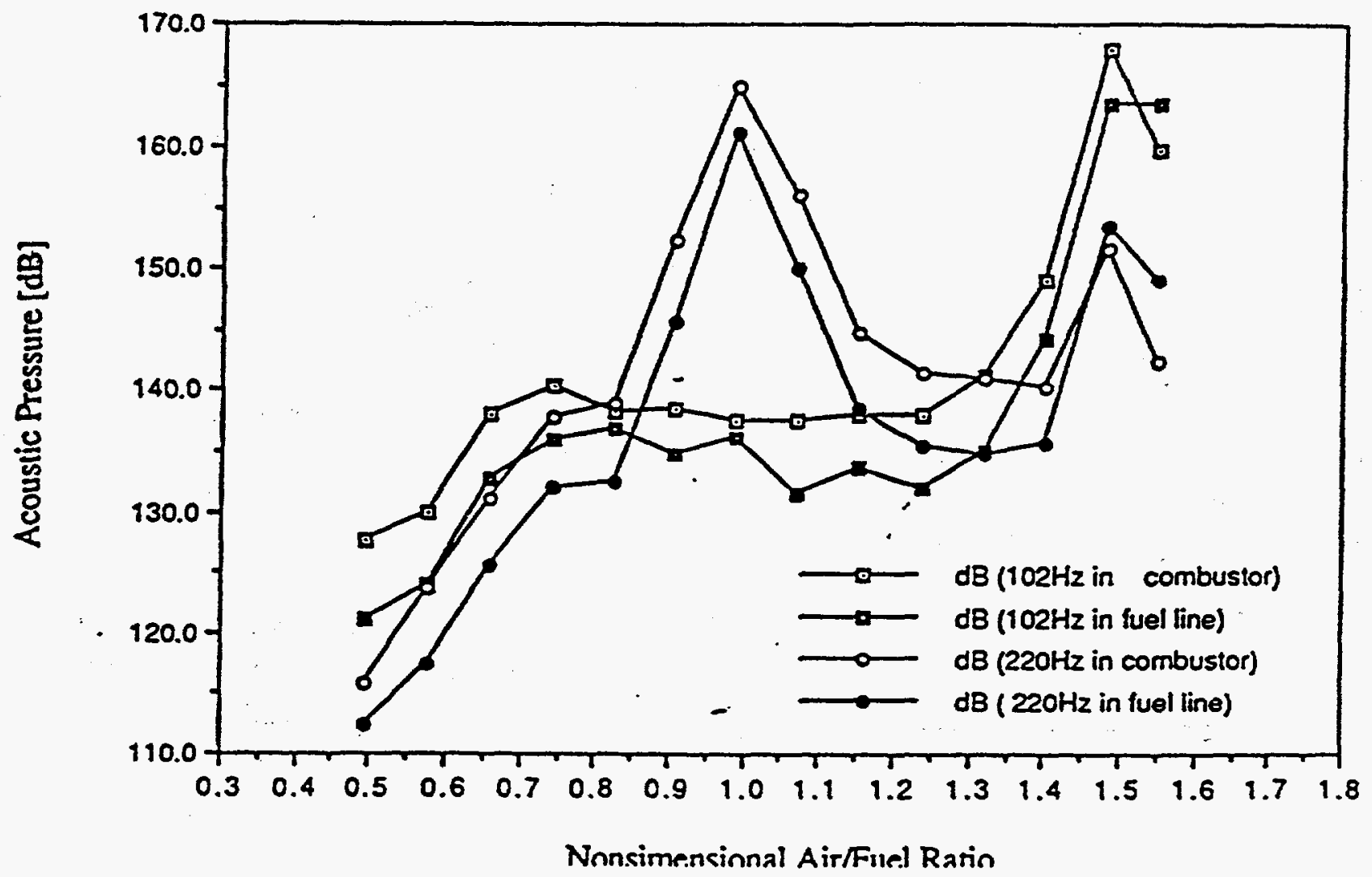

Fig. 39 Dependence of the amplitude of pulsations of the combustor fundamental and first harmonic mode oscillations and the pressure oscillations in the fuel and air feed lines upon the nondimensional air/fuel ratio measured in tests with 1.5 SCFM propane input rate and the regular injection configuration. 


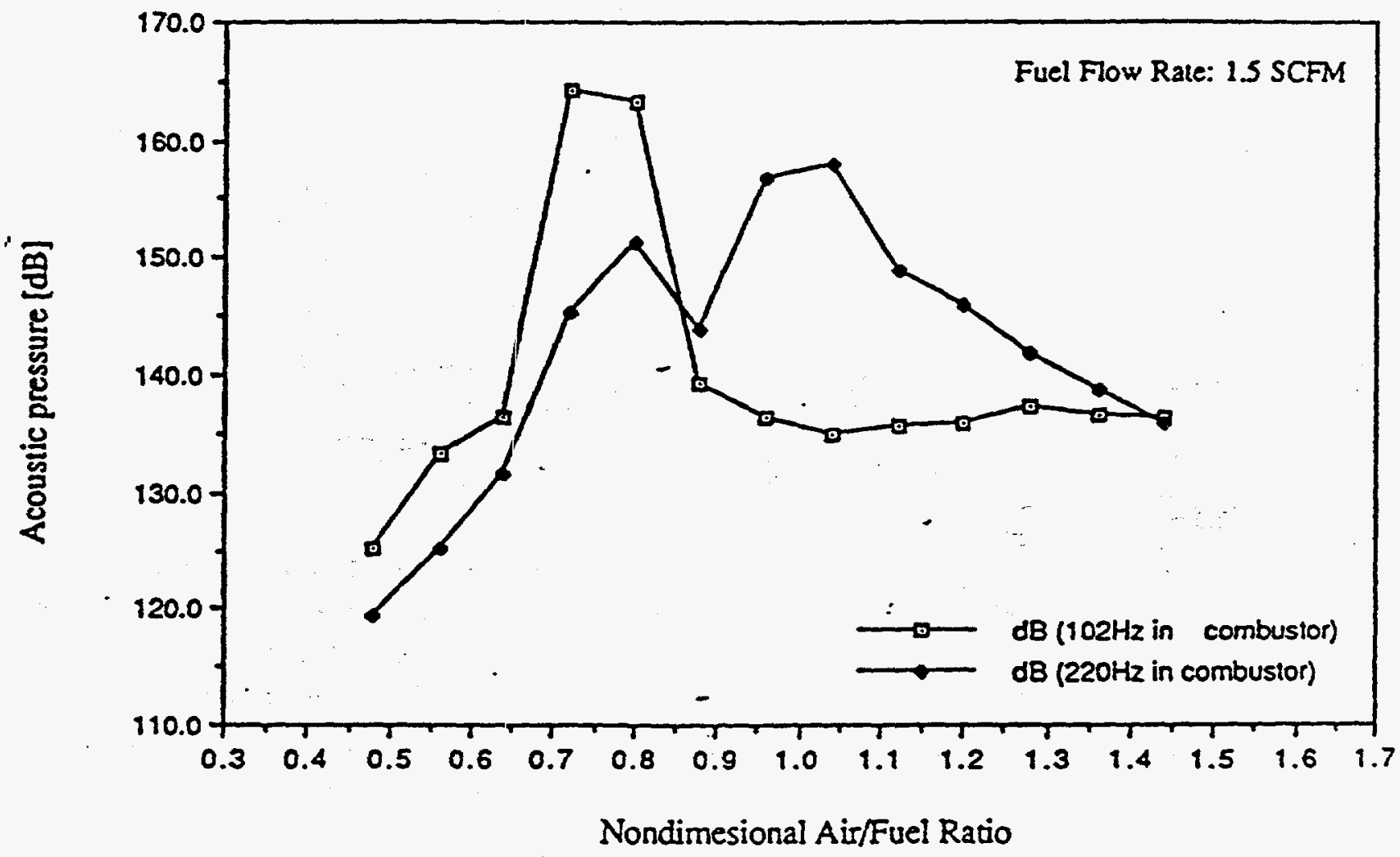

Fig. 40 Dependence of the amplitude of pulsations of the combustor fundamental and first hamonic mode oscillations upon the nondimensional air/fuel ratio measured in tests with 1.5 SCFM propane input rate and the fuel feed line chocked. 


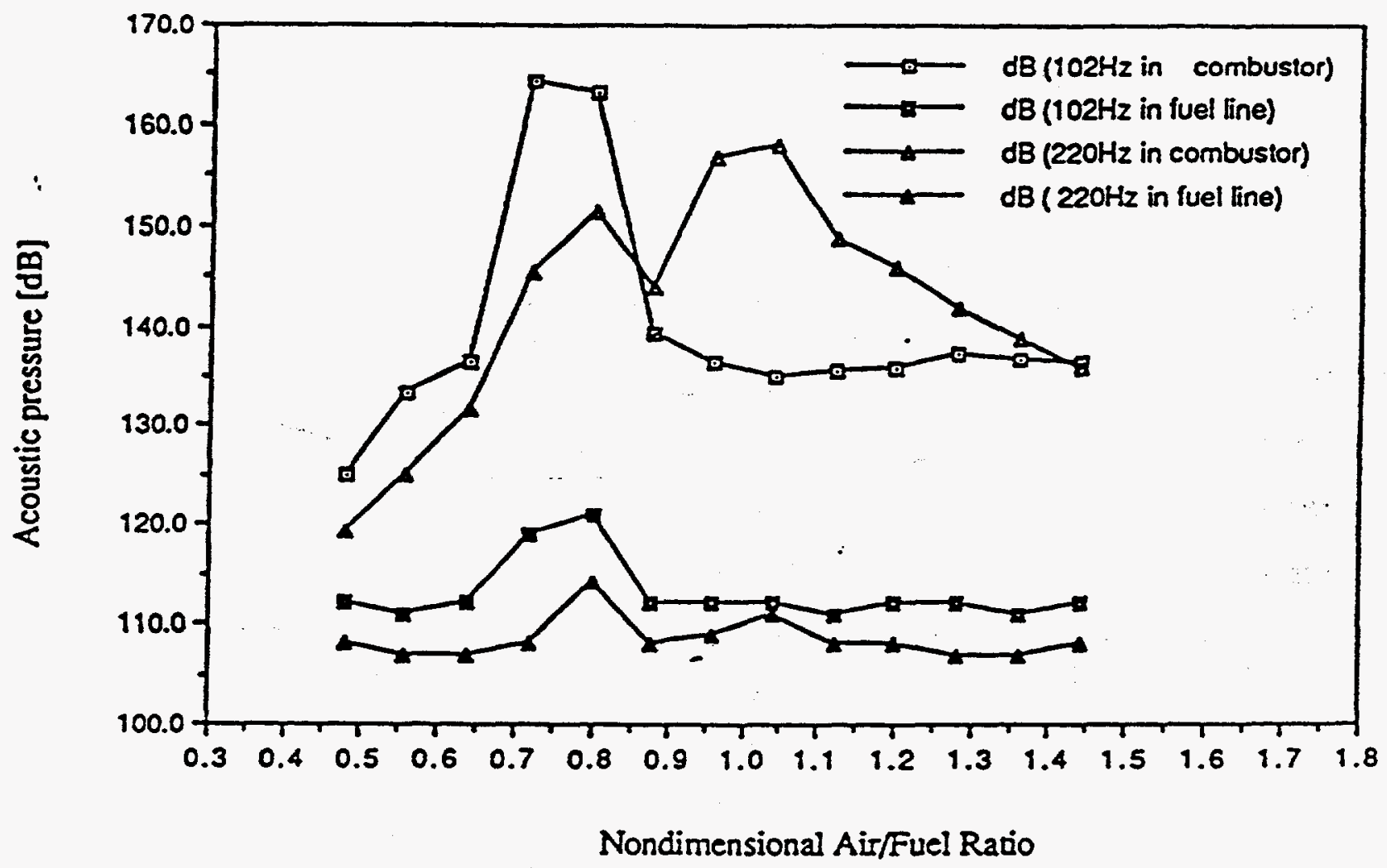

Fig. 41 Dependence of the amplitude of pulsations of the combustor fundamental and first harmonic mode oscillations and the pressure oscillations in the fuel and air feed lines upon the nondimensional air/fuel ratio measured in tests with 1.5 SCFM propane input rate and the fuel feed line chocked. 


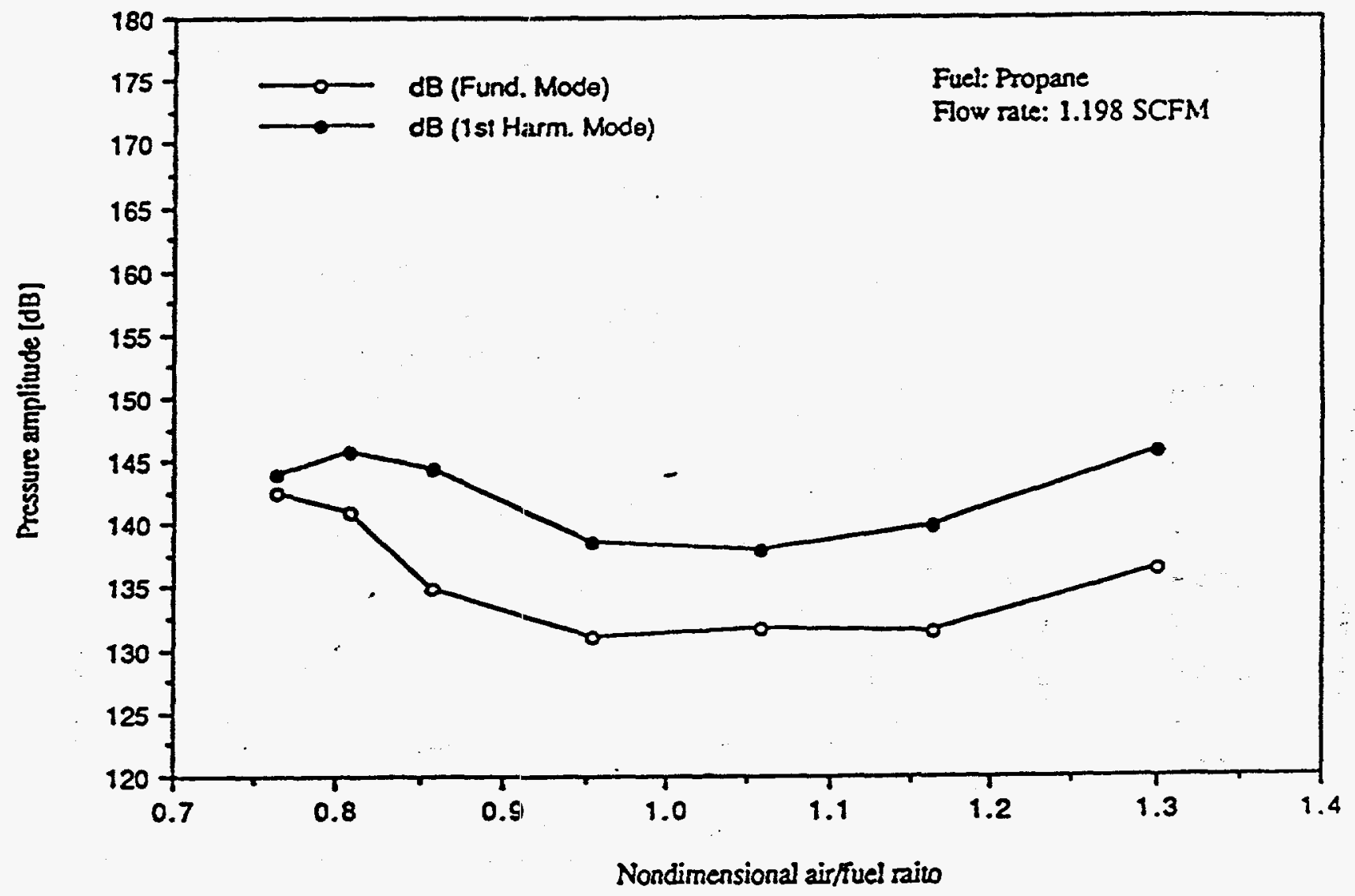

Fig. 42 Dependence of the amplitude of pulsations of the combustor fundamental and first harmonic mode oscillations upon the nondimensional air/fuel ratio measured in tests with 1.198 SCFM propane input rate and the air and fuel feed lines chocked. 


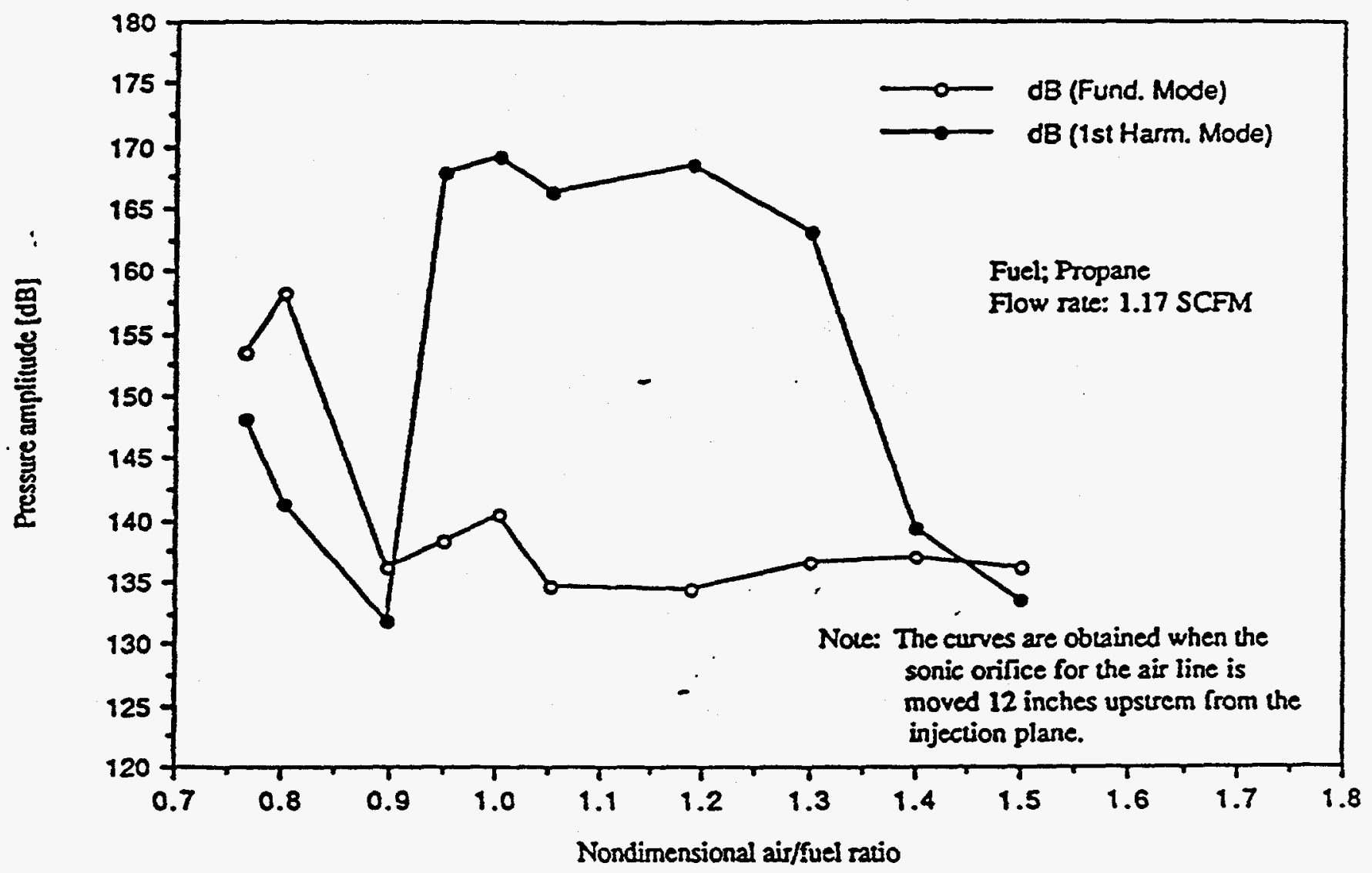

Fig. 43 Dependence of the amplitude of pulsations of the combustor fundamental and first harmonic mode oscillations upon the nondimensional air/fuel ratio measured in tests with 1.198 SCFM propane input rate and the air and fuel feed lines chocked. The air line orifice was installed 12 inches upstream of the line outiet. 


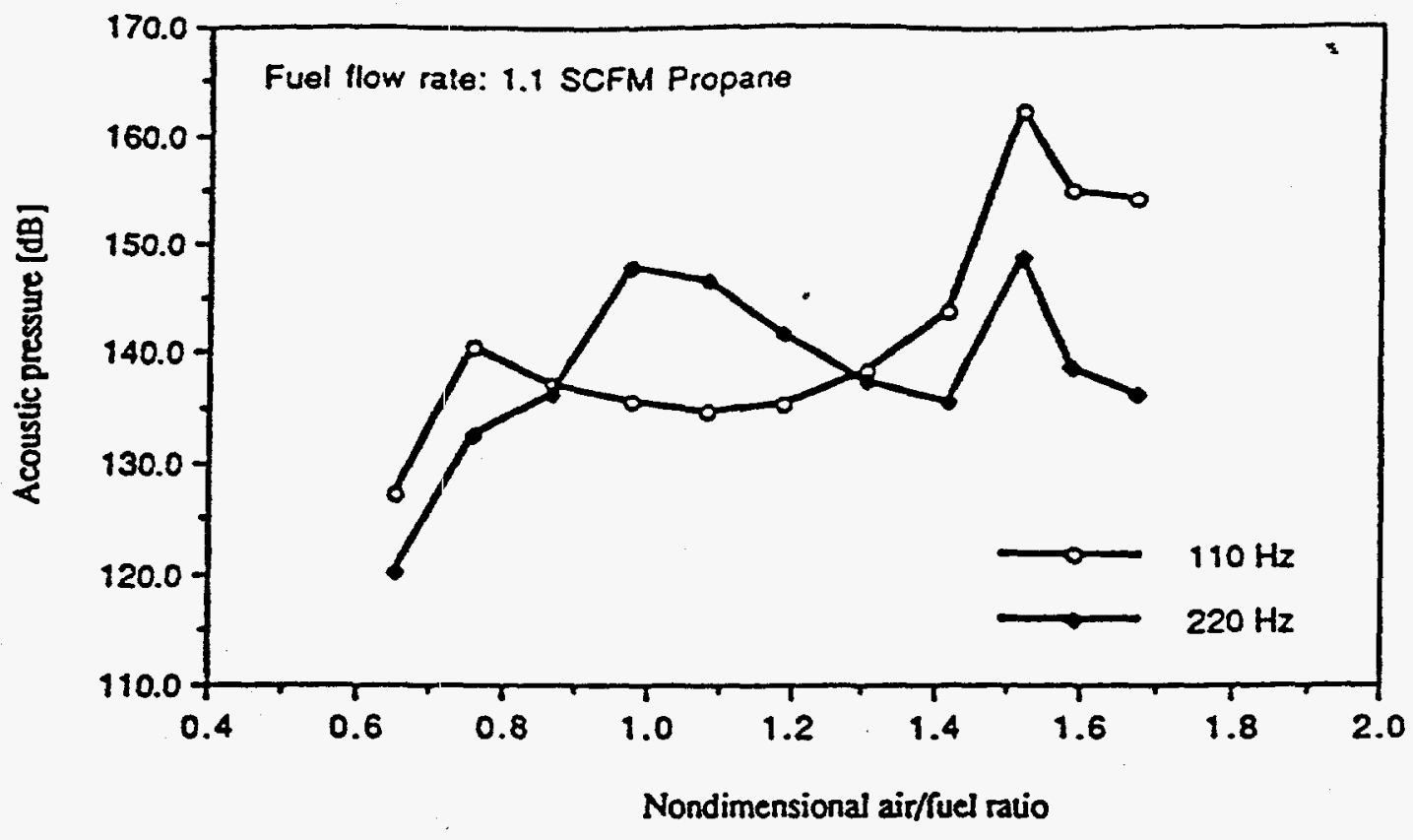

Fig. 44 . Dependence of the amplitude of pulsations of the combustor fundamental and first harmonic mode oscillations upon the nondimensional air/fuel ratio measured in tests with 1.1 SCFM propane input rate and the regular injection configuration.

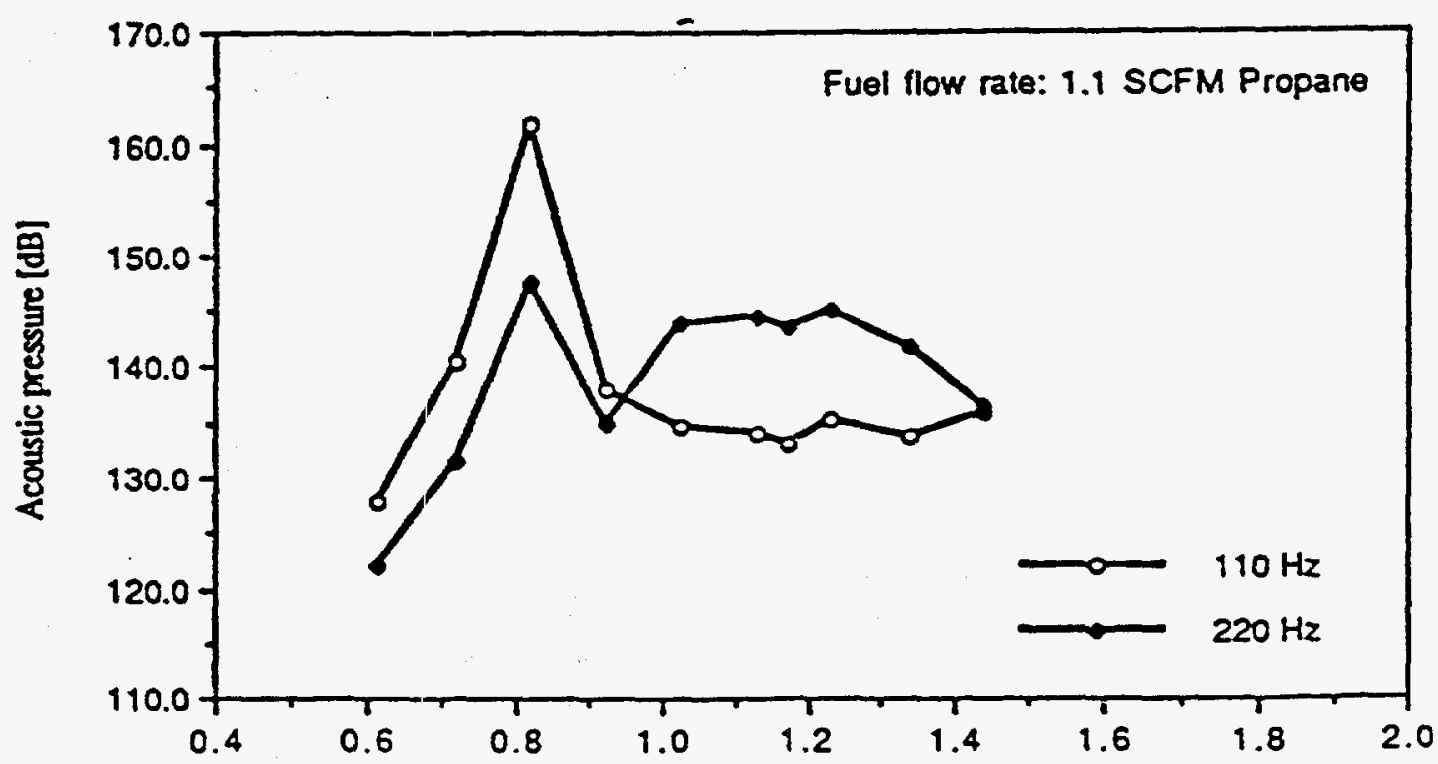

Nondimensional air/fuel ratio

Fig. 45 Dependence of the amplitude of pulsations of the combustor fundamental and first harmonic mode oscillations upon the nondimensional air/fuel ratio measured in tests with 1.1 SCFM propane input rate and the fuel feed line chocked. 


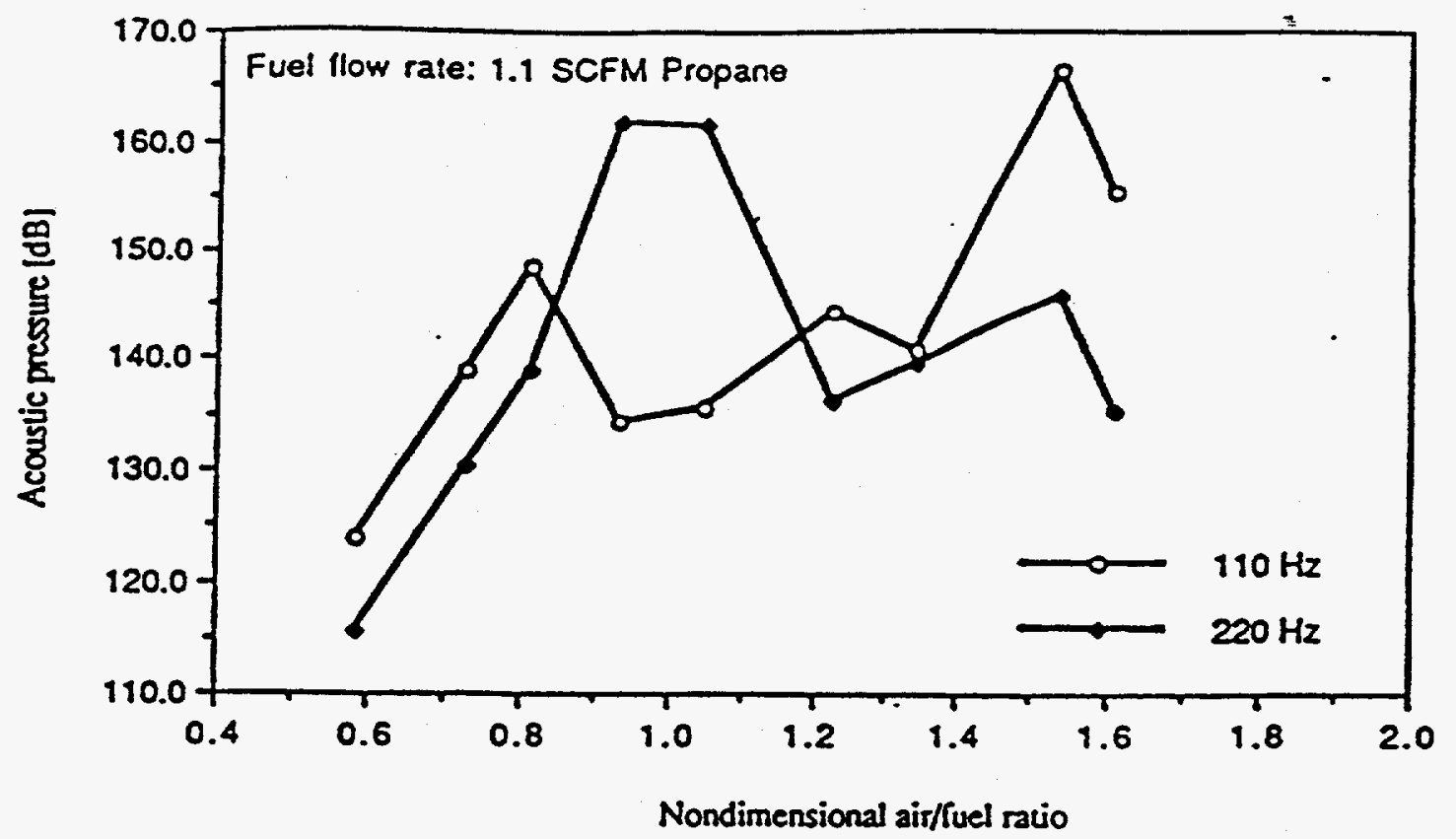

Fig. 46

Dependence of the amplitude of pulsations of the combustor fundamental and first harmonic mode oscillations upon the nondimensional air/fuel ratio measured in tests with 1.1 SCFM propane input rate and the air feed lines chocked by the modified impingement plate air injector.

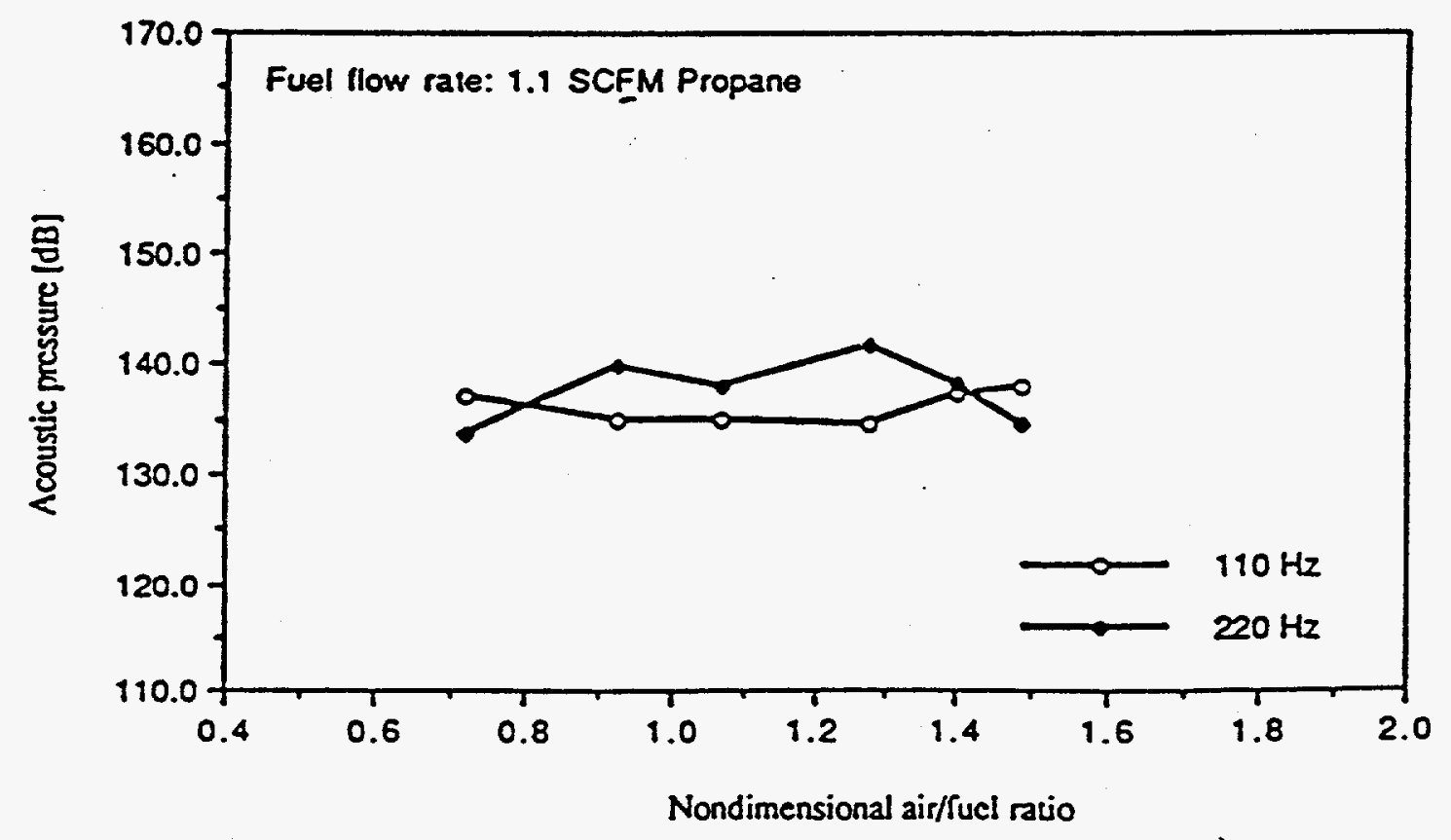

Fig. 47 Dependence of the amplitude of pulsations of the combustor fundamental and first harmonic mode oscillations upon the nondimensional $a^{i}=/ f$ fuel ratio measured in tests with 1.1 SCFM propane input rate and the air and fuel feed lines chocked. The air feed line was chocked by the modified impingement plate air injector. 


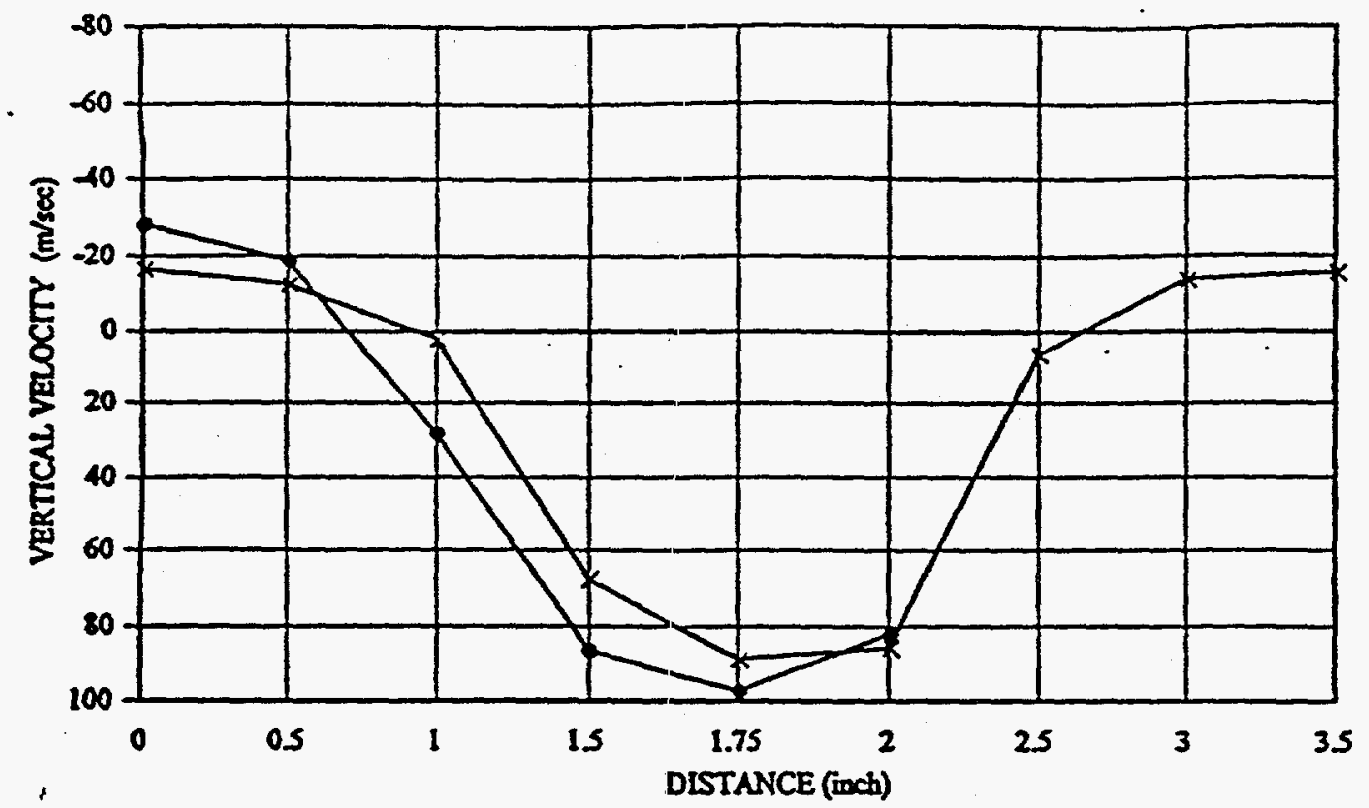

Fig. 48 Vertical velocity distribution across the injection duct at 2.5 inches from the injection plate. Positive velocities towards the combustor pipe, negative velocities back towards the injector plate.
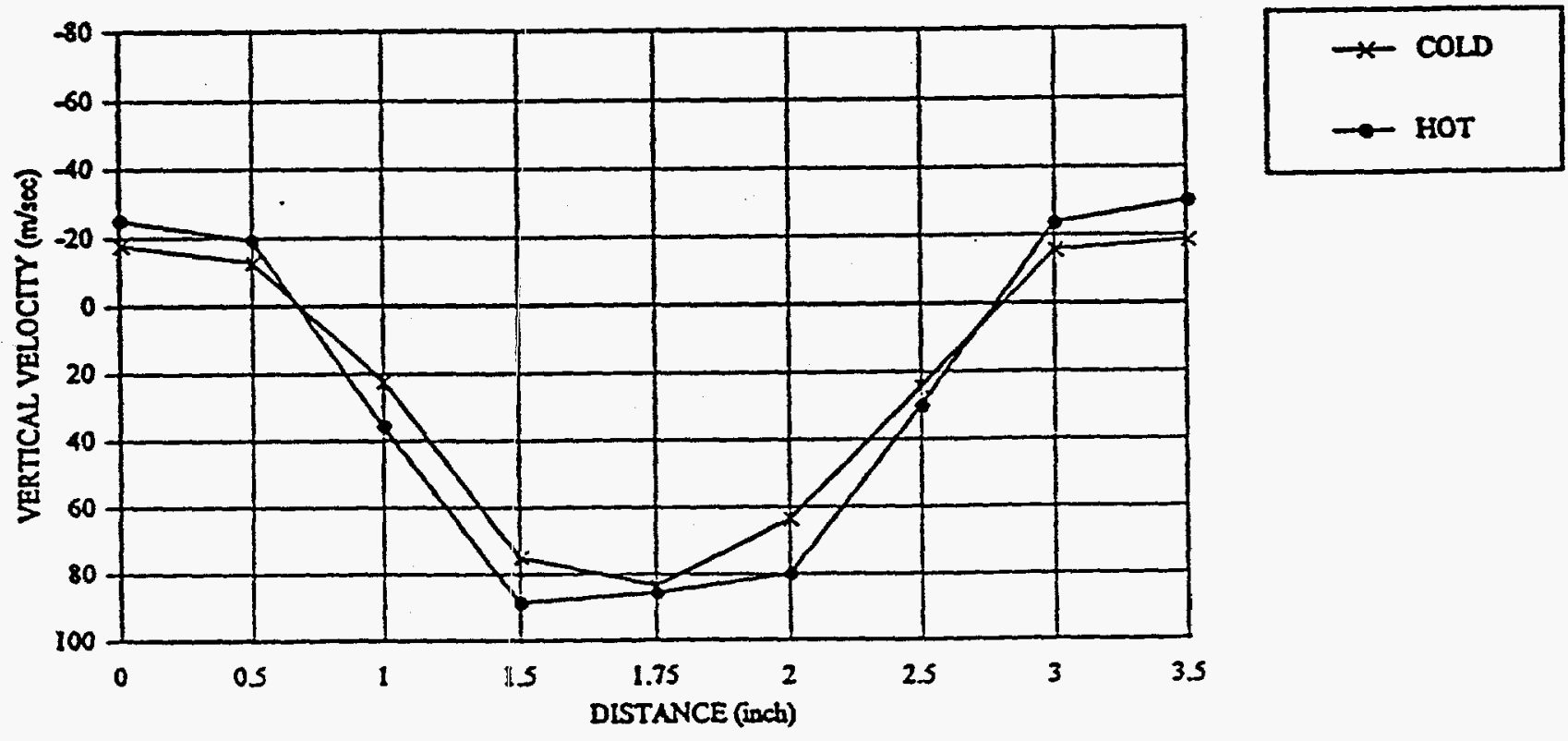

Fig. 49 Vertical velocity distribution across the injection duct at 3.5 inches from the injection plate. Positive velocities towards the combustor pipe, negative velocities back towards the injector plate. 